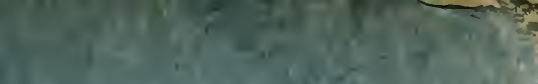

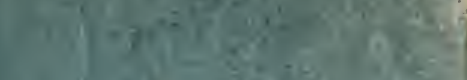

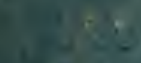

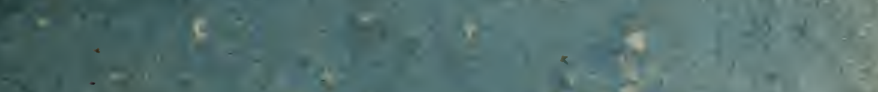

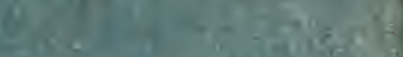

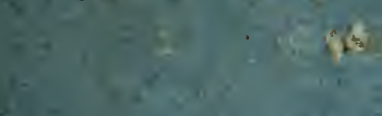

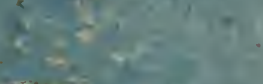

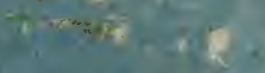

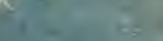

7

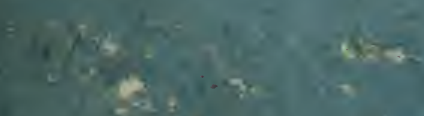<smiles>C1CCCCC1</smiles>

in

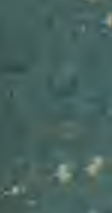

$$
\begin{aligned}
& \text { - in } 3 \\
& \text { ?. }
\end{aligned}
$$

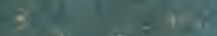

$$
\begin{aligned}
& \text { is }
\end{aligned}
$$

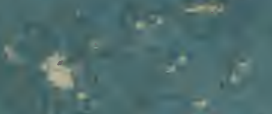

$\lim _{9}>-1$

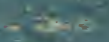

$10+2=$

*. $\quad+$

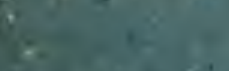

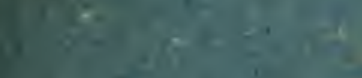

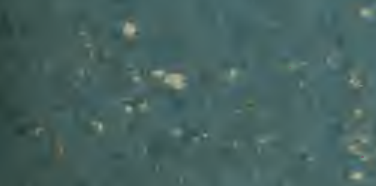

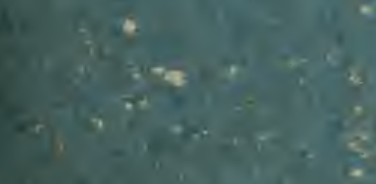

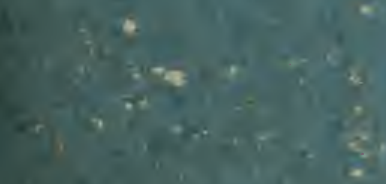

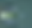

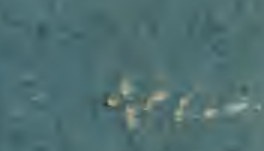

$$
\begin{aligned}
& x_{x \rightarrow 4}+4
\end{aligned}
$$

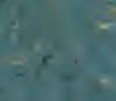$$
-v^{2}
$$$$
x+2
$$$$
\text { + }
$$

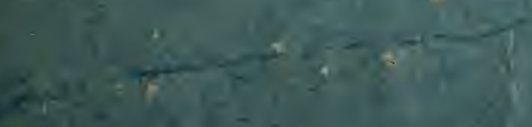

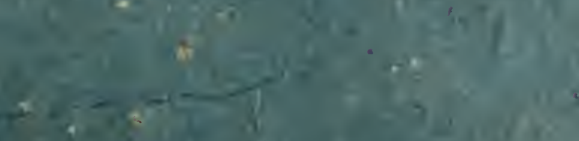

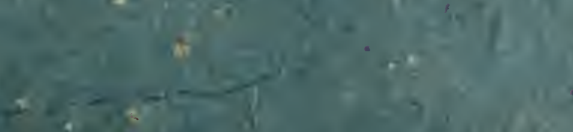



2749 



$$
\mathfrak{B} \in\{\text { d } \mathfrak{x} \text { e } \mathfrak{i} \mathfrak{b} \mathfrak{u} \mathfrak{g}
$$

Der

(S) $\mathfrak{a} \mathfrak{t} \mathfrak{e} \mathfrak{n} \mathfrak{a} \mathfrak{n} \mathfrak{a} \mathfrak{g} \mathfrak{e} \mathfrak{n}$

; น

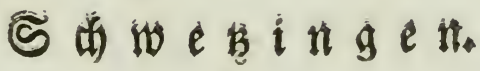




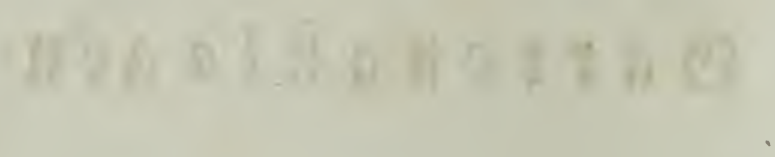

axwintoras 
Digitized by the Internet Archive in 2010 with funding from

Research Library, The Getty Research Institute 


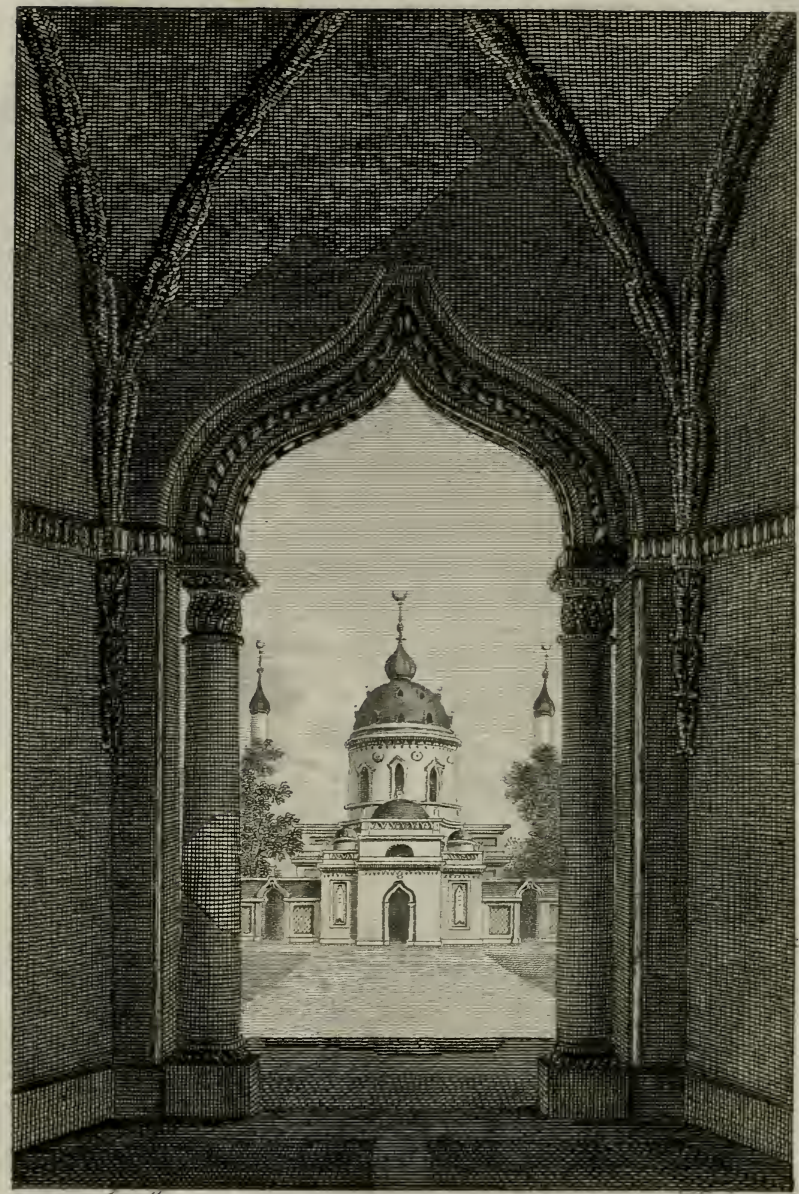

yow. unn yrull

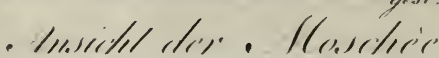

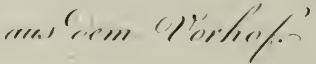




$$
\begin{aligned}
& \text { - Pisiderivilenel }
\end{aligned}
$$

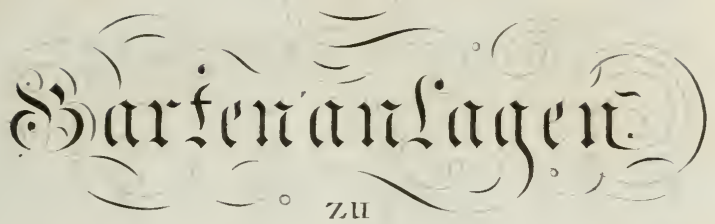

$$
\begin{aligned}
& \text { SCHWETZINGEA }
\end{aligned}
$$

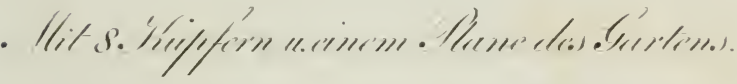

$$
\begin{aligned}
& \text { tw } \\
& \text { - Yreverrescyeysteren }
\end{aligned}
$$

von Gartendirector Zevher und G. Roemer.

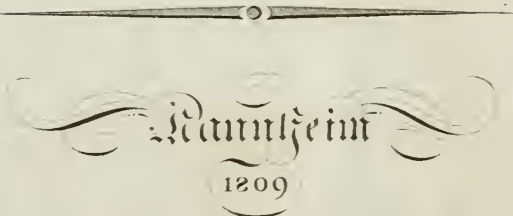

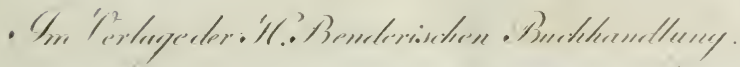





\title{
St. Sơniglichen șobeit
}

\author{
Dem
}

ourdhludutigften Firften uno Şerrn,

\section{$\mathfrak{R} \mathfrak{a} \mathfrak{f} \mathfrak{f} \mathfrak{i} \mathfrak{e} \mathfrak{d} \mathfrak{i} \mathfrak{d}$}

(5) robfergoge vou Baben, f̧ergoge

$$
\text { iu 3ägringen } 2 c_{\text {。 }}
$$

bem Neftor ber Şertider,

bem erbabenen Sefdither ber Sưfite uno $\mathfrak{B}$ iffenid)aften,

Dem Dater Des Datertandes;

weifen biefes soert

in tiefizer efruturde

Die f̧eraugeber. 


\section{(1) $)$}

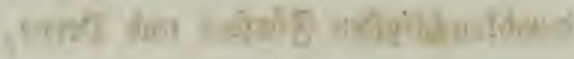

\section{(10)34531781759.}

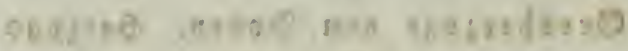

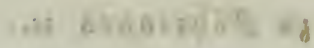

\section{-}

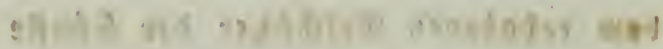

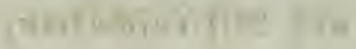

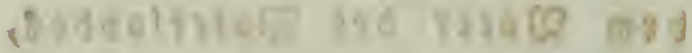

las righ that

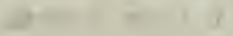

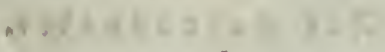




\section{$\mathfrak{B} \mathfrak{D} \mathfrak{x} \mathfrak{e} \mathfrak{d}$}

So mertwurbig aud ber Garten z̆l Sdmes fingen ift, und fo båufig ex zal jeber Zeit bes fudjt wurbe; fo ift bod) niemals ingeno cine

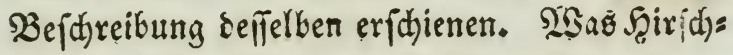

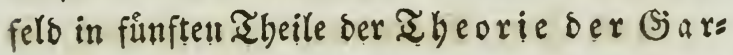
tentenft, S. 344. Davon fagt, ift theils za unvollftánbig, um eine ridtige Soce von beur Garten zll geben, theils find feine $\mathfrak{A n}_{\text {niichten }}$ nidjt imner bie ridtigften gewejen, wie wir in unjerer Darfitellung zu zeigen gefudts buken.

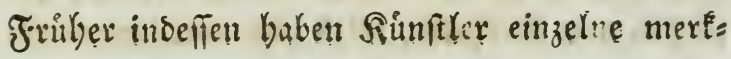
wưrdige Partbien bes Gartengs aufgefáft, uno

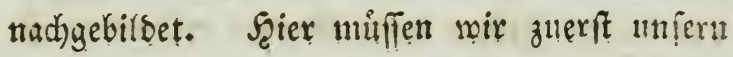

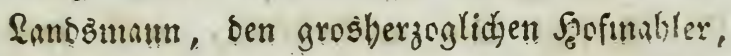
Sart Sun

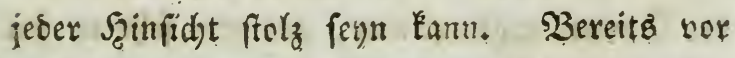

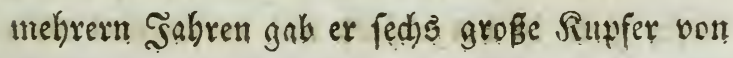
Yarthien bes Sdjụef̧inger Gartens herans, 
Sie fo grofenen Beyfall fanden, baf bald alle 9 the bricte bergriffen waren. Da nun feit biefer Deit mandes in bem (Sarten fich) verånbert und berifuonert, und ser Geift bes Fiunftlers nene Anfidyten gefunden bat: fo hat Şerx Runf̧ fid entid)loffen, bie fdon chebem bearbeiteten Gjes genfitinoe neu aufantelymen uno zu fedjen. Zwey Şlatten find jest fertig, bie wir gefehen baben, nno bie wir fulbn alb Meifterwerté empfeblen exnnen.

2(ud) Şerr Rottmann, ber jüngere, in

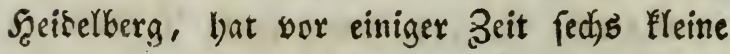
WYatten geliejert, weldye bie borzinglidyften Theis Ye beś Ģartens barftellen, uno bemerkt zu wers sen verbienen. Fin Eleiner, ungefelgr biex 3oll grof̧ex splan ses Єdumefinger Gartens, ges fiodjen won slerberft, wirb bier nux barnm an= gefilbrt, weil ex bas Exfte zu fenn fdeint, rags vor vielen Salyen voin Ginten erjojienen iff. (Fr ift jef̧t nur mextwirbig, meil man Earaus fielyt, in weld) falle.tem Gefdumade ser Siarten angelegt roax, und wie febrex feit biefer 3eit ift vergrobert uno vexidjonert wore ben. 
Inbem wix mu bem Publieum sie volls

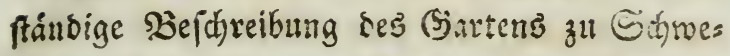
Esingen ubergeben, minfen nir yor allem bie beilige $\mathfrak{P f l i a j t ~}$ ธeร Danfez gegen jene Freunde bes Sdjunen unb Ġuten exfüllen, die uns in wiffenjdyaftlider uno artipifider Şinfidyt unters fitist baben. Der Serr Şofeammerrath und Gefalluerwalter Beller in Edyefingen bat

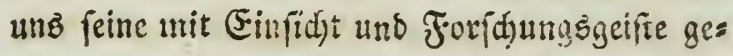
fammelten Miaterialien zur Eefdidyte Sdjnes f̧ingens uno ber Gartenanlayen mit freunbli= der 3 ereitwilligleit mitgetbeilet.

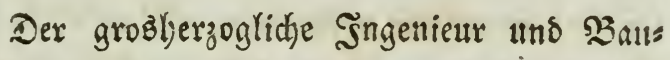
Infpettor, Şerr Dyuterboff, ber Sobn, bat die

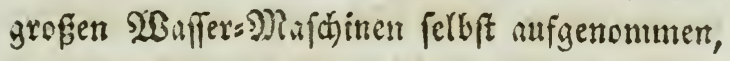
sie 3eid)ungen savon mit eigner Şand entwors

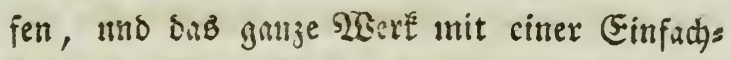
beit uno Sejtranigkeit befádrieben, weldye von jeinen ausgebreiteten Renntniffen zcugt. (5:

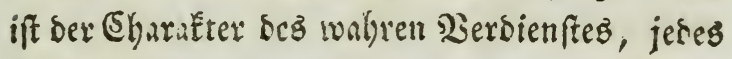

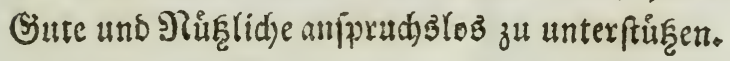

Sndem wir biefes fajreiben, erfalten wir

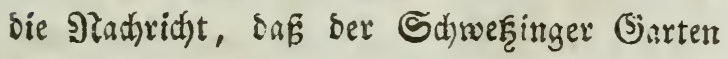
burd ein unerelárhares (Exeignif eine feiner 
grofften Zierben verliert: bie bimmelanftreben= Den Mlatanen, bie zum $\mathfrak{T}$ beile ủber finfzig Salve blibten unb ourdy ibren ftolzen $\mathfrak{W}$ udd feit mebrern $\mathfrak{S} a$ hren allgemeine $\mathfrak{B}$ ewunderung erregten, merfen ibre $\mathfrak{B}$ !åtter $a b$, uno ver:

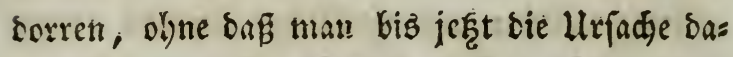
von bat entoecten Ksnnen. Der SBintertálte faun man biefen 2 erfuft nidjt zufdreiken, ba bie jungen ein bis vierjålbrigen Platanen vors trefliti) fteben, und felbft bie breysigjåbrigen an niebern Srten nidjts gelitten zul haben 1deinen: allein an etwab bod liegenden Stels len find aud biefe veroorben. Man wirs nut alles. genat unterfudjen, und die Refultate in

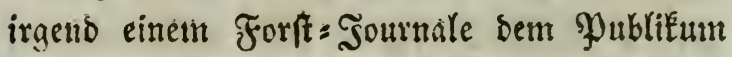

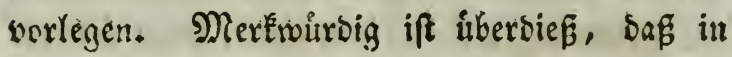
ben Şofgarten zu Sartsunbe ebenfalls alle atte Platanen berdorbent find; um fo mebr verbient aljo biefe jonberbare Exjajeiming unterjudt ju werden. 
$\mathfrak{R}_{\text {ein }}$ Drt in ber $\mathfrak{W}_{e}$ lt ift po flein und unbedeutend, Daj jeine (Befdichte nid)t irgend etrons MerEnürbiges Darbëte, und Erimerungen an vergangene Sage wedte. Der Mreníh Eefort fo gern in Die Borgeit zurüf; benn nid)t felten ftöß̈t er auf Ereigniffe, Die

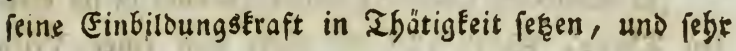
oft fein Şerz erwărmen.

Sit reifhen fich an diefe Bilder verflofiener Zeiten theure afngedenten; unt fo wirb dem Meniden ein

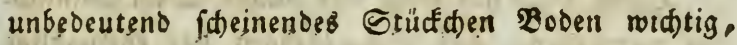
und er rúfumt feinen Rinbern unb Zeitgenofien die verğdwundenten Iage der Săter.

Darum niro es sem Sefer nidft unwillfommen

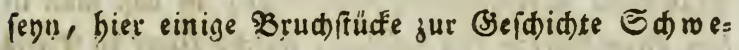
kingens ju finden, ebe wir ifn einladen, uns us

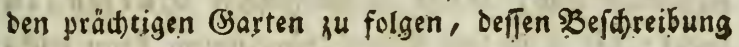
wir hiermit bem \$ubliEum übergeben.

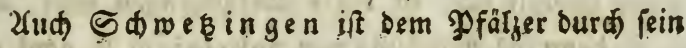
alter efrwirbig; fein Uriptung verlieret fich in bem Dunfel ber Borget, uno erft vom Sabre 1350 an

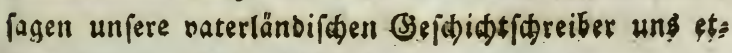
wos beftimmses darüber, 
Marquat Freber nent es Guejzingen fuper Aluvium Suarzaha. *)

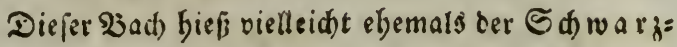
bad), und nad) ifm bätte bann wobl ber Dit felbft S d) wärjingen Eännen genamt worken jegn. atuein Marquard Fred)er fareibt Suezzingen; im fünf̧ebnten Jabre der গiegierung Fipins ( 767 ) farieb man diefen Namen eben fo, uno rer Codex Laureshamensis bat unz cine Gd)entungsurfunce sez Raifers Rarl, deş Jieçen, ocm Sabre 782 auf: bewabit, woris man Suezzingum fintet.

In oemfelben Codice Laureshamensi if im brit. ten Sheile, Geite 176 die Viede von either Villa quae dicitur in Sozinga, uno in oer Note, a) bie Semerkung: forte Suezzinga.

Daber lieje finds wobl mit einiger $23 a b r$ abeinlith:

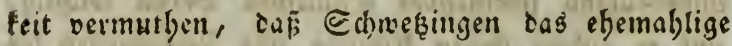
Solicinium genefen (ey, no bet Sinifer Salentınian Dell Allemaunen eine Schladt geliefert bat. **)

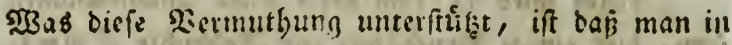
ben alten Siarten wun Sanson, melde 1650 zu Paris eridienen fino, Etatt Sdwezingen Suezingen findet; das e in tem Srgute Suezingum Earn leidb) feiner Giefralt naú in ein 1 ubergegangen fevn, uno fo ift bann dic zibleitung von Solicinium fo ganj uns

* In orig. pal. Lib. I. Cap. VII. de pago lobodunensi. Rheinhardi rerum pal. in script.

**) Amm. Marcellinus L. XXVII. 2. 368. 


\section{$-3-$}

mabrideinlid) nidft. Daß̈ übrigens die Rëmer it Diejen Gegenden geneien finto, berweijen die Denk. mảhler, welde bie Zeit nod) nidjt zerftört bat, uns ven benten balo ausfübrlicter bie siebe fern wirb.

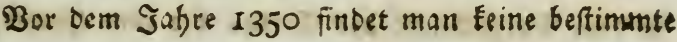

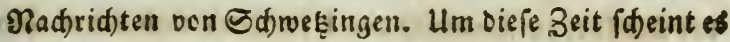
eine Eamitie ven Sdomberg beferfen zu baben, oon

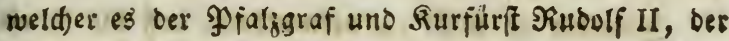
1353 frart, erfialten baben ford. *)

Im Sabre 1412 foll ber Surfürt unb PFalzgraf

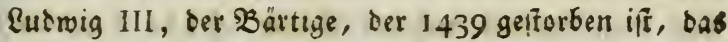

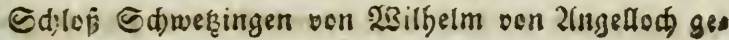
Exuft baben.

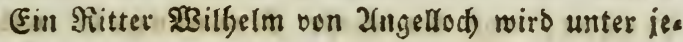
nen genannt, weld)e sem Surfürften Friebrid) I folg: ten, als er WSadjenbeim an ber Şaarb belagerte; ifgn nennt aud) Siremer im UrEunbentudbe zur (5)

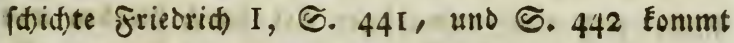
Dieter, ber Junge, von angellod, vor.

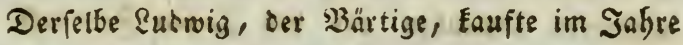

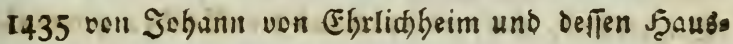
frau für feine zweyte Gesnablinn, Matbitie, Iod, ter des Grufen almabăus voll Savoyen, cillen Jgof in Sdjweşingen.

*) Christoph. de Clingensberg processus historico-juridicus in causa Successionis palat. Ingolstadii I I I I. S. I 24. "Elisabetha Schom"bergensis arum suam Schwezingen Rudol"pho II, comiti palat. coscedit." 
Christ. de Clingensberg 5. I27. „Johanaes "de Ehrlichheim et Uxor. ejus vendunt Me"thildi Sabaudicae curiam in Schwetringen." 2lud) einen গIupedt von (Fbrlidbeim findet man in tem 2 sergeid)niffe der Eoeln, Die bem Şeeresjuge Des Surfürften ช̛riedrid) bes I folgten, uno unter ifm in ber Salnd)t bey Secfenbeim fadtes.

Indeffen if nid)t zu vermutben, Daf bie fo oft vorEummenden Saufe und Sertaufe immer das ङd)lof betroffen baben; es ift vielmebr bödhft mabridbeinlich, Dấ mall Daınals mebrere (Sitter Şäfe (curias) nannte; und roirtid) werden mande nea) jeß̧t fo ges

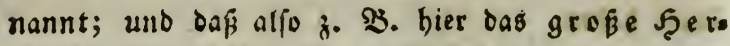
fengut, uno das grepie Nonnengut, weldes fpäterbin an bas Stift Neuburg gefommen ift, gex meint feyen.

Das Sdló 5 dwebingen war ebedem. befe= figt, unto Hach Damabliger 21rt ourd) Gräben uno 3ugbrutten gegen femblidbe anfälle geiithert. In mebreren UrEunden aus ben Beiten Friebrid, Des Siegreid)en, witb er ourchaus bie $\mathfrak{B}_{\text {eft }}$, genannt. In bem Seftamente feilles 23aterb, redurd) ifm folde nebft ber Sandoogten im slfaffe, gemeinia)afts lich) mit feinem jüngern Bruber, bem Serzege アius pert, zum Erbtbeile beftimmt wurde, beißst es bis Sefien Sdweringen und Sirfdgarts. ba uien.

Rremer (jefhidte Frieorid) I. S. 6. 5. 3. In einer anbern UrEunbe, 5ribelberg uf \& ritag nad. 
Et. Sincengien $=$ Iag (Den 24 ften Sanuar 1472) worin gricorid) feîfferzet, was gefhehthen folle, wenn (Er jïh vermäblen uno Erben befommen foate, überläft $E_{r}$ feinem Padfoolger sic Santregter) im El: faffe, barunter $S_{\text {dr we }}$ ingen, bie Defte.

Rremer 5.454.

In Demfelten Sabre Eaufte ser Surfürft friedrid einen Barten bey bem Edille Edwetzingen, unt

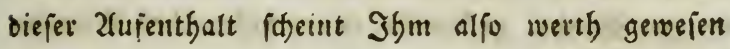
ju รern.

Son bem Sdleffe felbit fintet man nid)t bie mins befte Madridt); nirgends it eine Suri, welde ben Fericher leiten fỏnte, ben Zeitfunkt der Erbauung zul erratbert.

In einem Eleinen Bebäube fieft man auf einem

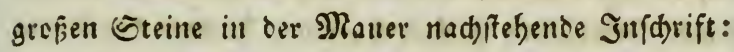

PFALTz GRLVDWIG CHVRF. I54I.

Dies ift ber Namen des Surfürften Suowig, bes Friebfertigen, welder fechs uno breyfig Sabre res giert hat. Do $\mathrm{Er}$ aber ber eigentlid)e (Frbauer bes Sdbutżinger Sdleties geweien fen, Eann nidjt mit seffimmtheit behauptet werdell ; ter E.tein mit ber angefüfrten Inidjrift ift an einem alten Nebengebäus be, und mag wobl einit cine ancere Etelle gebabt

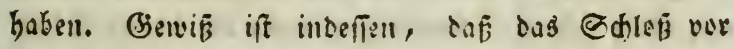
bem Jabre 1544, uno jwar ven elnem fraligrafen unt Siurfitrften ift erbant wersen. In iem \$hurme redter Şano ben bem Eingange findet man in oem 
zuesten und britten Stode auf ben juey Sdfufifreis

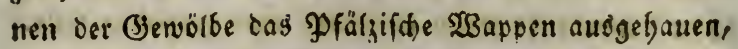
befien oritter rotber 2 Bappenfdild ned) leer uno obne Den SReidbapfel iff. Diefe 2fuszeidunng erfielt erft Surfürit Friebrid) II. aus beiondever 2sergünfigung Des Saifers Sarl V. auf bem Reidstage zu Spever 1544. Diefer Reidjappel erfdeint aud) 1547 auf cinem sbaler diefes Siufürfen, weld)en Söfler

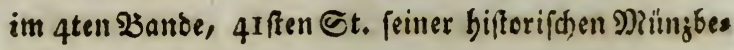
ruftigungen befareibt.

Son nun an fören alle Nadridtent ven bem

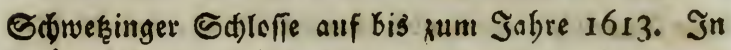
Der bey Sjottharb sögelin zu Seeibelberg in biefem

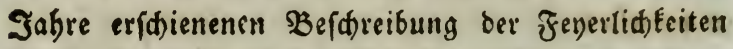
Bey Der ZurücÉEunft bes Surfürften Friedrid) V. mit feiner (Semablinit Elifabeth, einer Soditer ves Jöni= ges JaE ob von Englano, Keiftes im 26ften Sap. \$.198:

„ ङ\$ Gat \$Gro EGurfürftlide Binaben auf ein Meil

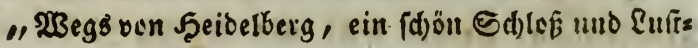
ibaub, Sdwefsingen genaunt, babey es ein ftatto "lide und febr grofe 2 Sillofubr ven J̧edwild in

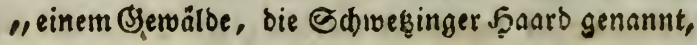
"fo fid) auf bie zwo Meilen erftrecft."

Im Sagre I620. brad) ber Drenbigiäbrige Srieg mit allen feinen Sdrectniffen auş; bie fhône SFfals warb von bem panifonen Benerale Spinola und bem Saifertidjen Gienerale Silly erobert und verwultet; im

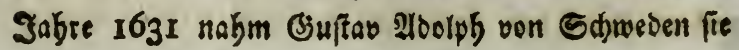




\section{$-7-$}

in Sefik, und vier Gahre fpäter nabm ber faiferticte General (3allas lie abermals cin.

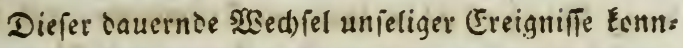

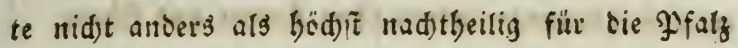

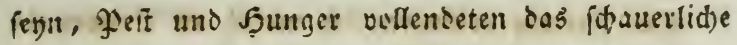

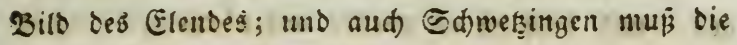

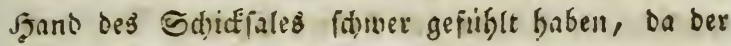

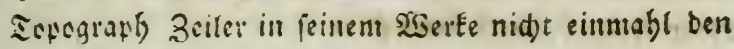
Namen Edwex̧̧ingen nennt.

Diejer idrestlide firieg warb I6 78 durd dell bes rüfmuten weishbälifhen frieden geencigt; ser Sitrs fürfi Sarr Sutwig, Der Gobn deß unglüftliḑen Fries brid) V., trat dic Diegierung an; mit ifm begann

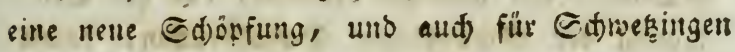
cifnete lid) eine fidshere BuEunft:

2(us einer B̉eid)reibung, die ber Şeffereiter Şans Fard) im Jafye 1650 vom Edwef̧inger Sdleffe eins

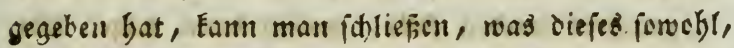
als Edjweşingen jelbit wäbrento ier Beit ber Stürme inuf gelitten batien.

Das 厄dics war mit cinem breiten (5raben ums geten, über ten eine mit $\widehat{E} d$ iefern getecte $\mathfrak{B}$ rüufe

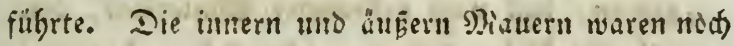

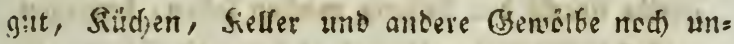
beițäbight. 2ffein in Barbofe war bes fieflets 23 cbuba us gam jerfiert.

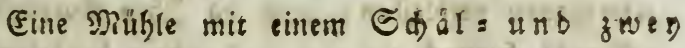
Maghgangen; 


\section{- $8-$}

die Piortenfube unb ber Pfortentburm,

oer Marfd ftall, fwengrobe Sdeullen, brey $\mathfrak{B}$ iebeftälle lagen in Erummern; uno bie zwey Biefbrunnen warell obne Setten und Fímer.

Sart Rubwig erinnerte fid entid an Sdinersing gell, uno beichlós es wieter aufzubauen, weldes nadffehender Beridt bez Siellers Ejedias \$Rai vom $x 7$ ten 2 uguft 1656 heweift:

"Demnache Shro Surfürftl. Durd)laudtt vergan, "gene $230 d e$ als ben 13 ten, Itten unt $15^{\text {ten }}$

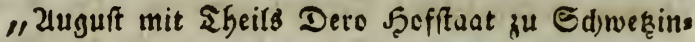
" gen logiett, untewn Şödiftgebad)t Şbro Sturfürftl. "Durdl. nir gnäbigft befoblen, Daf zur Bele=

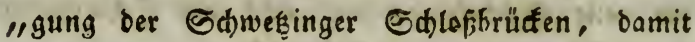
"man aus. und einfabrent E⿱onne, ebeftens Zunfalt "gemacht mủrbe; weilen im $23 e r k$ ein $T$ beil bes

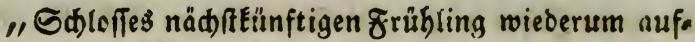
"geridtet werben folle, mafien bann wegen 266 . "räumung felbigen इbeils, als oud) die 2(uśfübs "1 rung bes Summers im innern Sdloshof, Shro

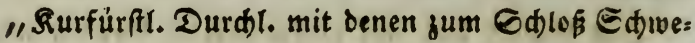
"Gingen gebörigen frobnoörfern allbereit fich ser= "gliden: baß́ nad) vollenbeter 23 interfaat, Die= "felbe ftradt's ben zinfang maden follen; bingegen "alles Eifenwerk, als aud Defen und Steine, fo "in felbigen Begiré fid) befinden würden, ben Uns "tertbanen verbleiben; aud ba \$̧̧ro Surfürft. "Durdl, bernat sin eber bas andere benöthigt, 


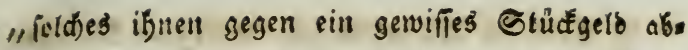
1) Eaufen, voer fie es aufferbalb zu verEaufen Madt "Gaben jollte."

Im Früblinge bes Sabres 1657 begab fid der Surfürif mit ber Barcrinn yon Degenfelo nach Edweęingen, und zu Ende bes Şerbftes nad grans Eenthal, wo fie den 5 ten Dftuber I658 ifren erfen Sobn gebahr. Bad ifrem 230 denbette fübrte fie

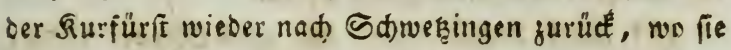
bent gröften Theil ibres Sebens zubracte uno Muts ter von ad)t Söhnen und fünf Södtern wart.

Den fefern wiro es̀ nid)t unangenefin jegn, bier ein Serzeidnif ber Sinter z̆ finden, weldhe der Fur=

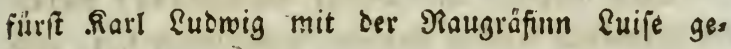
jeugt bat.

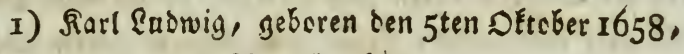
gefterken ben I2ten 2fuguft 1688 .

2) Saroline, geb. Den Igten December 1659,

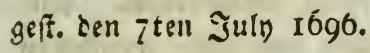

3) Euife, geb. Den I $5^{\text {tenl }}$ Sänner I66I, geft. den 6ten Fe5ruar 1733 .

4) \&udwig, geb. den Igten februa: I662, geff. ben 28 fiten Măr 1662.

5) 2(malie Elifabethe, get. den 22 fen Măr 1603, geft. ben I 3 tell July $I=0 \mathrm{cg}$.

6) Beorg fubrig, geb. bell Zolien Miär 1664, geft. Dent Ioten July, 1665 .

7) Friederike, ge5. den 27 fîtn Jung 1665 , geĩ. ben $=817$ in suin 1674 . 


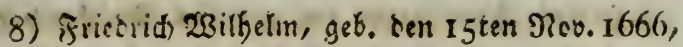
geit. Den roten Iuny 1667 .

9) Siarl (Eouarb, geb. den gten May 1668 , geft. cen 2 ten Sănner 1690.

(10) Sunbie, geb. ber gten July 1669, geft. ben I8ten Nov. I669.

II) Siarl Morił, geb. den zoften December 1670, geft. Sen 3 ten Suniy 1702.

12) Sarl ?luguft, geb. den gten DEtcber 1672 , geff. dell Ioten Geptember 169r.

I3) Sart Safimir, geb. Den 22ften 2lpril 16\%4, geft. Den I8ten 1 pril I69r.

Diefe berzlide und innige Sserbindung, woven die Biefdidte wenige Sepipiele aufzuzäblen bat, jer.

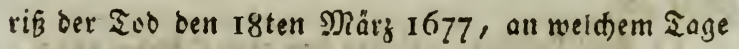
çie eble raugräfun Suife zum beflern \&eben "̈ber= ging; aufridtig bellagt von bem erbabenen Siarl Eubwig, uno geebrt von iffen Beitgenoffen. Sirl \&ubrvig befd)lok bie (Etbauung einet allen driftidien Sieligicnen, welde Deutihlands Staatsgejęce begüns figten, gemeinfd)aftliden Sird)e in ber Jeltung frie: brid)6urg ju Mannbeim. Er gab ifr ven Mamen jur beiligen Fintradt, und in biefem Sempel forte feire gefiebte Ruife ruhen bis jum Iage tes Qizhtes. Er ging den 24 ften Mläry früb uno obne alles Siefolge auf ben bazu beffimmten Yloz und fing an mit eigener Şand an ber Grundage biefer sobs tengruft zu arbeiten; dann entwarf er fariftid den 
FIan unb sronete bis Reidenfener, welde aud) kes reits ben $3^{\text {ten }} 2$ furil Nad)ts vollingell ward. Der füblente Rejer wiro dicje Eleine 2lbiveid)ung vergeifen, und diefes erlauchten Faares andenfen fegnen, bas nie erlëfchen wirb. -

2lus ien in sen Aften ber Rammer befinolichen sigenbäncigen Jicferisten des Surfürften Sarl \&ub= wig, uno aus ben $\mathfrak{B}_{3}$ iijungen ber Siechenfammer an Den Furîneifter Sabne, an ben Forîtmeiffer Dber. rbeiner, an ben Semmandanten und Dberfen ven Mienges, an sen Befälloerwefer Sdomitt zu Seibers berg und an cas Banamt, vin dem Jabre 1657 bis 1679, erbellet, daż verfd)iedene Beränterungen uno

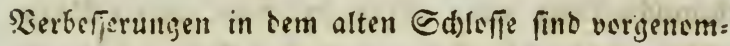
men worten. 2lllein feit jener Zeit bat 8.35 巨d)lö eine ancere (jeitalt erbalten, und es ift nid)t möglid ju beftimmen, weldye seränberungen uno wo foldje find gemaht werben.

Den 25ftell Dituber I680 ftarb Fart Ruimig, und fein Gobn und Taubfolget, Marfürft Sarl, crs

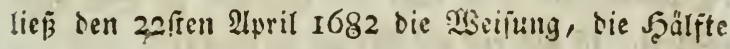
ber in bem Surfürfflict)en (Barten ber friedrid)sburg Eefindtiden Bitronen = uno Poncrirgenbâume nat) Sd)wetzingen 3น bringen.

Diefer Sirfürft Sarl fơ)entte burd) eine ben 22 ften Jebruar I68r auสggefertigte UlEunte feiner (jemablinn

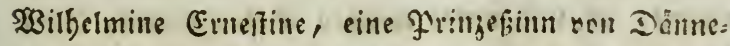

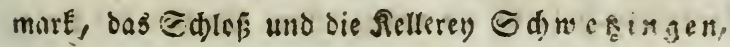


Fent के diweningen genant, mit allen baju ges

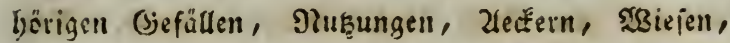

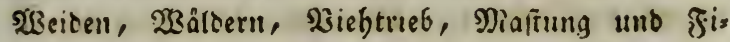
fherey auf oie Zeit ibres Lebens. Der Nachfolger

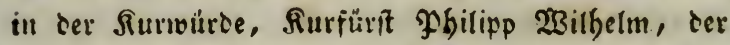
ben aten September 1690 z̧u Wien gefterben ift, bes ftätigte biefe Gdenkung f $^{u}$ Seeitelberg ben 27 fiten DEtober 1685, und beffen Silnn, ber Surfürft ฐo: bann W3ilfelm, zu Neuburg an ter Donau ben 26 ffen fefruar 1691. Surfürff Rarl ftarb den I6ten Dian 1685, und nach feinem soie brach ber unfe=

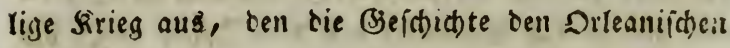
Erbfolgetrieg nennt, unb ser fo verdertlich fint bie rheinifde Pfal wat. Man hat Feine anbere Nad)s

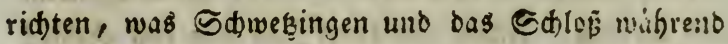
diefer Sdreçengzzeit gelitten haken, als einen $\mathfrak{B} e=$ richt bes Rellers fu Weriau, Ehrifteph Mayer, vem 28ften December 1699, worin er fagt, "तaf "bas Sitibes uno Melforeybaus von bem fralliofificten "Branto unverfebrt ftefen gebleben, die Rübe uno

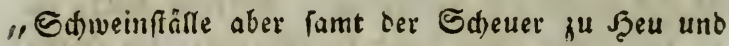
"Strch, ift bis auf bie Maner verbrannt."

Fad) (Encigung biefes für die Mibeinffald po ver. Derblidjen Sirieges, befabl der אurfürft Jebann 2 sil= belm ter ŞofEammer burd) ein Nefeript, Sd)werzin, gent ten 27 ften Ceptember 1702, cinen seriud) ju madsen, of unb unter welden Bedingungen, Shre

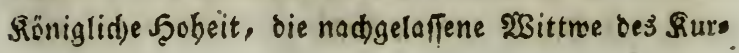


fïrften Ratl, bie bisher ju Ridtenburg gelebt batte,

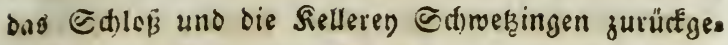
ben welle. Dieje lluterbandlungen batte Eeinen Eis folg, unb bie Surfürftinn blieb in Befike biefer Domäne bis ben 22ften, 2fpril 1706, no fie ftarb,

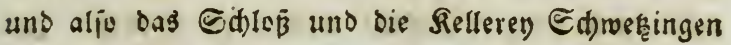
wieier an Das Sutbaus jurüáfieren.

2in einem Steine bes alten 5ळloffes liep't man

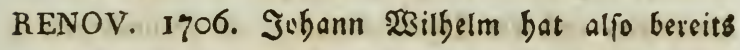
im erifen Sabre nach Dem Esce der Jurfürftenn (Er:

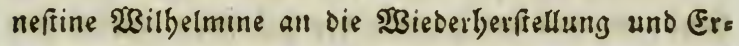

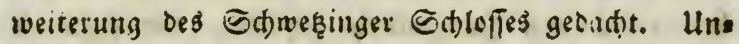
wiberfprechlid) gebören ber 2unbau gegen ben (Sarten und bie bevoen flügel des ๔alofles in bie regies

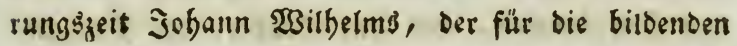

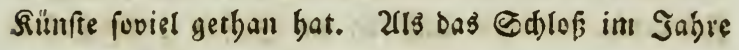
I774 mit গ⿰氵士Setterableitern verfeben rard, und man bie alter Fabnen oon ben Sbürmen regnabm, fanb man bie Jabrzahl I54I mit einem Blech jugededt, und bafür 1708 singegraben. Der Siurfürit Эebann

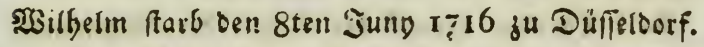

Sein Bruber uno Nadbolger in Der Sur, Siart Pbilipp, bisher Eaiferlicher Ctattbalter in Sirol, begab fid im frübjabre 1720 nad (Sd)reģingen,

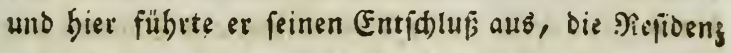
uno Dir fämmtlichen DiEafterien ven Şeide!berg nark Mannbeim zu verlegen. Sdon den 2 ten Suly $I>20$ legte Sarl \$bbilipp ben erften Btein zu Dem grefiess Refibenzid loffe in शRannbeim. 
Im Jabre 1722 beganı eine ineute, glutuflibere

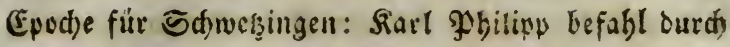
ein Piefcript voun 22ften Mlay jur 2lnlage des Cjars tens monatlich 500 fi. uno jugleid) 1000 fi. aus ben Jeftungşbaugelbern zu bezahlen, und bieje Erfọtber= niñe burd) antere Biefälle wieber zu erję̧̨en. Im Jafre 1726 wurien folon ju Folge eines Siejcriptes rom gten Sänner einige Felder jur Sartenantage ge= nommen, uno bie Eigenthümer bafür entỉhảdiget.

Im Šafue I732 mars das fogenamute biejantten= baus, welches ber framilie bes gefeimen Siathes und Reibarjtes ven sungwinth geförte, um 8500 fl. geÉauft.

Diefes Scatts bewofnten ber Şergeg CGriftian ven 3wetbrücéen, unb fein Bruser, ber Fjaligraf friebrid, weldser aud) da im Jabre 1767 gefter? ben iff.

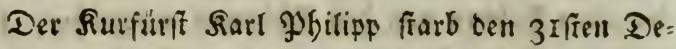
centber I742, im SIften Gabie, unb warb zu Mann= bein in ber Edslec = Sapelle bengefesct.

Siart Ifeodor ergrifi die Büget ter Siegierung,

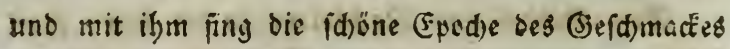

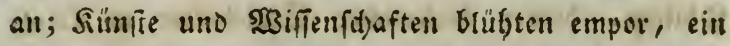
beiterer Iag war angebrodien, Der Borbote bes got= Denen 3eitalters. Ithe, Dentmäbler bes Stönen und (juten, bie uns nod) jeşt ungeben, entitanben in jenem merEnutresigen 3eitraume; oer Pfölger blicft nit cinem ebeds Sationalifolge auf fie, unb bentt

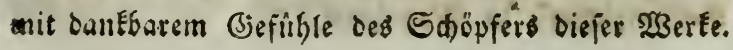


Rar! Sheocur wähite Edyweringen fu feinem be ftäntigen Sommeraufentbalte; or rief blübende Fflan. ¿ungen aus Eandourifen berver, und ber jedern (Sd)ritte fébit man auf Dionumente, Die jein (sefübl

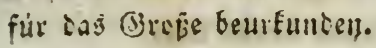

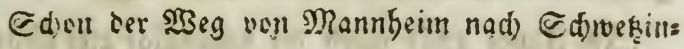
gen fribrte ourch reiţence Gärten, uno bohe भुas,

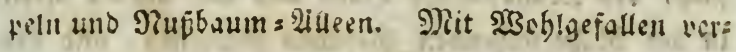
weilet ber $\mathfrak{B l i c t}$ auf reid)en fluren, den Suellent des allgemeinen 20 blifandes und des Heberfulles. Fin viers jebnjäbriger, unfeliger firieg bat grwar manthen biefer froljell sBiume gefüllet, mand) zauberifides (jepilde perwüftet; altein ber freundliche Şimmelêfrtrich be=

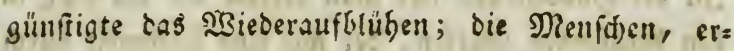
nürmt ron bem Giefüble für das Sdöne, pflangten uno bauten mit friidem Oiutbe; aus่ Srümmern fties

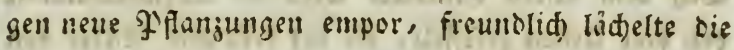
micterauffebende Fitur, uno belcbute ourd ibre

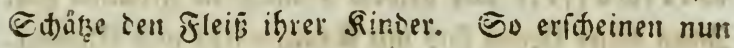
Dieje Ulmgebungen in einer jwar veränderten, bed nidft minder idjönen Bjeftalt.

Edrmeşingen felbff verfdönerte fid) numäblid); Der (Seift des erbabenen Beherriders breitete lid) aus ouf bie Sewobner, und fo fiebt man bier uno sors Frivatgebäude, bis sine Sauptitadt zieren rsurien.

Der Surfürft baute 1753 den Stall uns sie

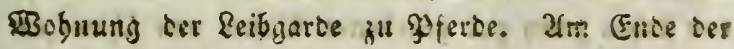

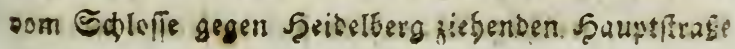


batte der Mfalggraf Friebrid) oon 3weybrincten, weto der 1767 ftarb, für fich eitren Marfofftall bauen laffen, weld)en Sarl \$lgeobor burd) ein Fefcript vom 15ten DEtober 1759, übernafm und bald barnuf die Flügel und bie Remife Datan bauent lię.

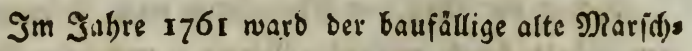
ftall, Des Munbbäcéer's Şaus und alle andere altent in der Näbe ftebenden Gebảute abgeriffen, und der nod) ieșt fteghende neue Rünenbau aufgefübrt. Son ben übrigen Bsebåuten werden wir am gebörigen Drte fpred)en; wir fübxten biefe wenigen bier an, weil fie unmittelbar bie anfidt 巨abwek̨ingens verfäönern. In ter Şauptftrape bat man bereits bie 2luafidth in Den berübmten Baarten, Der wie eine feenwelt burch

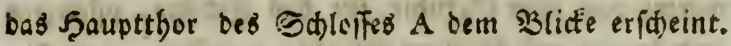
Bey Dem erifen Fintritte wirb bie ZufmerEfameeit von vier Urnen gefeffelt, bie bet beEannte ßritter von Derfd) a ffelt aus gelbem Saniffeine gefbauen hat.

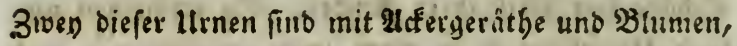

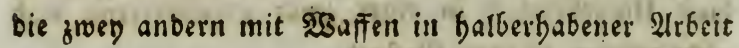
gejiert, unb beuten aljo wohl auf Frieden unb Srieg.

gididt unmerfiualdoig fino nod) auf biefer Etelle gwey Bildfáulen Diallens von $\mathfrak{b l e n}$; ein Speer und sin Sdyreingtepf fino bie 2(ttribute ber, meld)e rechts Stegt; Die auf ber limten Gaite hat Siöher, SYfoir, Begen unb cinen getöbteten Şajen. Nidjt weit das ven ftegen ziven gut gearbeitet Bafen ven fatwarjem Narmer, melde zu M?annbeim fino verfertigt morben. 
Gileid redits ftêfit mant auf bas int BirEel T748 gebaute Drangerte = Jiebäube, $C$ weldhes von geforues nen Eremen aufgefübrt, uno fects buncert હd)ub lang iff. ST 23 meter werden darm cie Drangertes Sônume aufberwatrt, uno, im Sommer fincet man Gier Edjut gegen die Etralen Der Sonne, urbeinen angenebmen Exarfiergang. Şier if auch ber Durds=

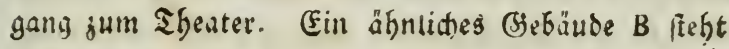
links ; ten 22 fren focrnuny 1753 wart mit dem Baus meiffer siabaliatti der Bertraly wegen diefes tinken Flügels geidloten, und ifm die Summe von 69000

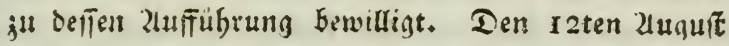
I755 war der Bau vollendet. Diefer Flúgel woro ju Speipe = und Spielfälen benükct, westn bobe zrem: de am Sacfe finto. Der Speifejal tif mit feinen, rethen unb weisen Steinplatten beleat; um Spleliuale ift ein Eünttid) eingelegter Boben von (Fid)entgol; , uno in benoen Sälen fint Samme von Marmer, sie Deffen und die 23 Bnnde find mit Ginksalbrit gejiert, unb unter bem Spielpale if ein geräumiger Sieller fll 2tufferwabrung von Ffianjen.

Sart Ifeodor wollte aud ber bramatifinen Mufe einen Sempel bauen, und befahl berests im Jahle

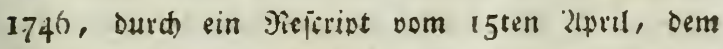
Dberbau = Direftur van Bibiena, etn Eteines Theater ju erridten, woz̧u die netbigen Mlatertalien von

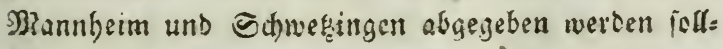

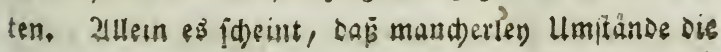


Jusfithrung ricjes sefefles verbintert baben, tas

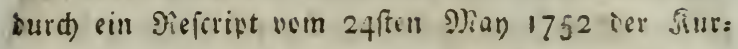
fürif feinen S⿱⺈ Des Dbertau= Direfters ren Fligage tas Sheater in bem redten Drangerieflitgel erbaut merten follte.

Nod) wirb biefes SGeater ven jetem Semmer bes wundert; ber গal:m if mit einer feltenen Einfidt

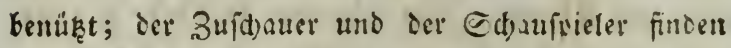
gleidhe Bequentidfeeit; bie $\mathfrak{B e r}$ ierungen fint einfad) unb gefd)macfooll; im Şintergrunte ser şübne if

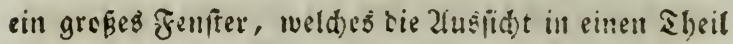

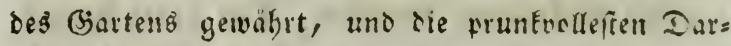

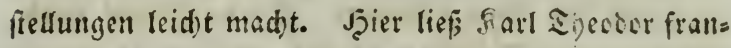

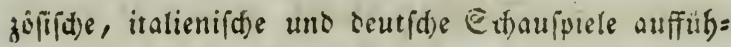
ren; woju fein SalE uno jeder gebiltete frrmoe den freyen 3utritt batten.

Im Sabre 1757, als Erllner Şafgäatmer war, entwarf ber Damablige 2aus : Intentant und nathes rige Dberbau = uno Giarten = Direkter son grigaige eis sen greben und Eubnen FIlan, Der ned serhancen

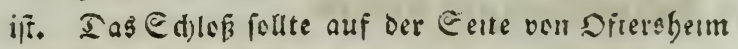
enweitert, uno ourd) mebrere mit grefen (jebauten umagebene Şoje einen Erifaunen erregenten limfung

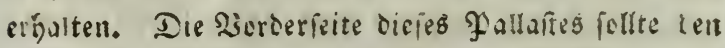

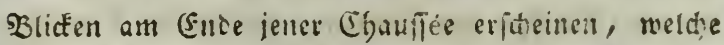

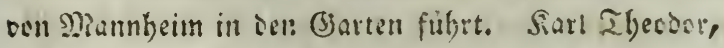

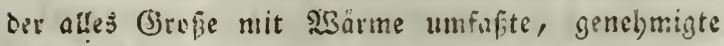
fogleid) siejen şlan, uno olye Beitverluft wurten bie 
Brunbmauern annelegt. Zallein entweder fant man Das :Bert in ber Jolge zu ausigebefnt, oser cer ulufs wano fdien ju gresi, ullo inun brach jum Ihetle die Giruncmauer wreier aus, oder fum Ibeile vaif malt fie miaber zu; und fo liegen nod) wutklich Diefe jun= Damente.

2ruch an bem Fnte des rects gefenden Drangerie: Saufes follte ein flügel angebaut werten, woonta)

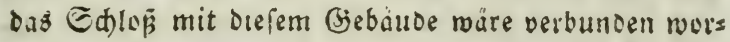
Den. Now jeigt man die Etelle, mo der siurfürit Sart Sbeodor ben erifen Eteun mit :telen Jenarita) Eesten gelegt bat. Dich ast(t) stele Joee ward nisht verwitetidt, uno die Girunomauern mutden eben: fulas wieder jujemerfen.

Inteiাan maro in cieiem ZeitpunEte cie lunlage des (3artens mit Ermife betreben. Sart Sbewser bes firmmte $\tau_{j} u$ iafjrltd) 66,000 f. Jian Eann Defien ungeachtet nibt genau angeben, was jeoes (jebarude

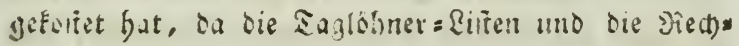
nungen Det Saunimet Eer uno Rieferanten ren cer (je= Mrakkafle kejuftt murten.

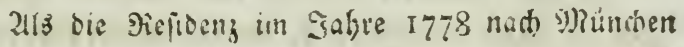
verlegt nurde, ferste man ebrige 5 umme ani $+5,000 \%$. uno ellolid) gar auf 24,000 fi. berab́.

Sil gerader Amie ron tem Saupteinange find

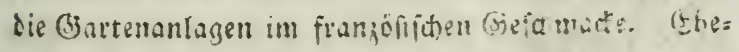

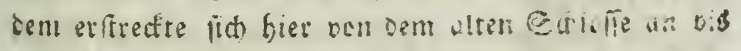

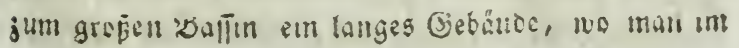




\section{$-20$}

SूSinter bie Drangerie aufberabarte. 2Im Ence degs felben war ein greper Gaal, der jum Epteien uno Evielen biente, und ten man deșegen audh bas 23 allf a 4 s nannte. Eabald aber tie beysen balbs zirfelfïrmigen Drangerieflugel erbauet waren, wurbe jenes Giebăude niedergetifien; es̆ waro cann en bes

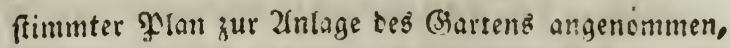
unb nach remperben fortgearbeitet. Sater fallen zuerit Das im MittelpunEte liegente grofie Saffin a und vier

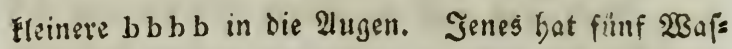
ferftralen, beren mittlerel, melden ein Defwbin,

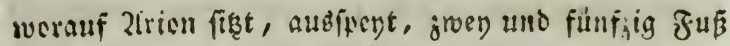
fi(i) erbeft, uno fiuf Zoll bick t/t. Die librigen vier Etralen fpringen aนs E(b) wănen worauf Kincer fik̨en

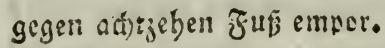

zlale bieje friguten von $\mathfrak{B l e y , ~ w i e ~ a u d ~ j e n e ~}$ in Dem $23 a f i n d$, nid) fern von bem Ferfen Pant uno bas fogenamte Eeepferd, find aus der \$erlafs

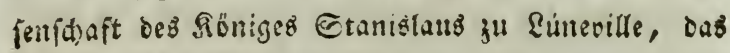
Pfund um ro Sous, gefauft morten.

In ben Eleineren $\mathfrak{B}_{a}$ fint find äbntide Sinders

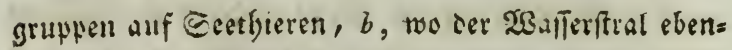
falls ađtjeben G(t)ub bad itergt.

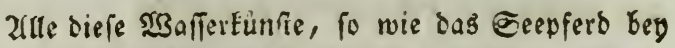
$E$, werten usn ber greß̈en Miafdine $N N$, getrieben,

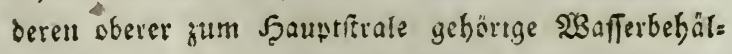
ter adit und jebentia §uf boch legt, und auf oeren voffintige sefdretbung wit zutükfommen werden. 
In einiaer (Entfernung erblicft man bura) bie

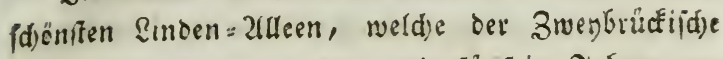
Scofgätmer Fetri vor brey uno fütffig Jabren ge=

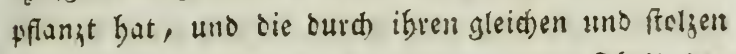

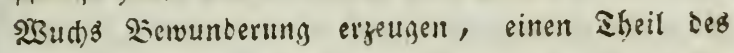

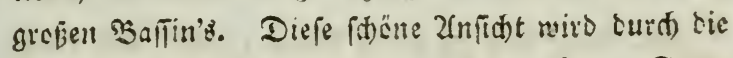
Sogelifaen Ssebirge begrannget, die it biauer Ferne (iid) erbeten.

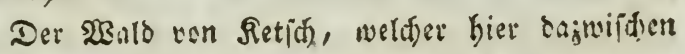
liegt, entjeg lange ben berrlidfen Inblice jener von

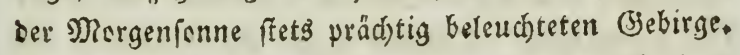

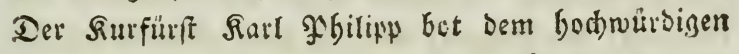

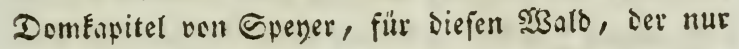

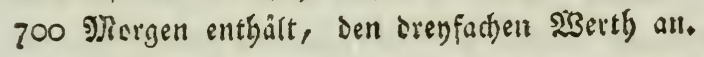

2ullein biefe Şerren wollten ifrem Seeiligen nidjto vergeben, und verlangten für das গ্乃n̈lod)en nid)t

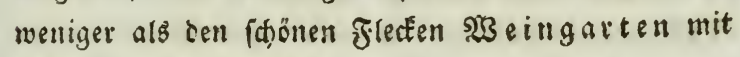
allem, was baju gefört.

Die Interfandlungen wirben aljo nathrtidier SEeife abgebroden, unb Sel Surfurft untertrictéte feinen Girall.

Im Gammer des Sabres 1720 werb Prilipps: burg von ben Gaiferlidjen belagert, und ber öfterreidi= (d)e Sieneral, (Graf von Sd)mettau, Eam nađi) Schroe kingen, cem Surfürten feine $\mathfrak{E}$ grffurd)t zu bezeigen. Sarl Ygilipl beflagte fid wäbrend ber Interbaltuna zufälig über bas po wenig nad) barlide Benebrnan 825

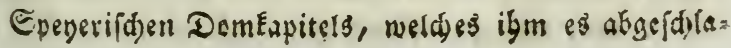




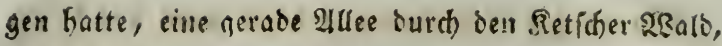
geaen Erfar, bouen ju lailen, um die 2tuefidt aus bem Sdwweķinger Ëhloffe iи crwentern uno zu rers

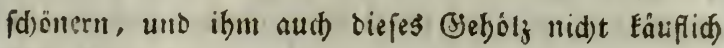
überlaffen wollte.

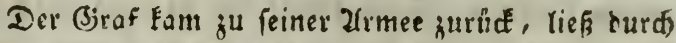
bie Eaiferlid)en Ingenieurs ned) in jener Nadt nad) Dem Merioian ven Єd)weringen, in geracer गird)=

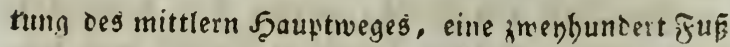
breite und eine balbe Etmibe lange altee abfectert, und ourdbauen. Darurch marb die edinbeit bes Bjarterrs, der ofnedies von rinigen Eeiten berefft iff, nid) weniq erböbet; und vor orey Gabren leé̈ Se. Sönigliche Sobeit, ber jeşt regiereriec (jrefpherzeg ven Saden, nech) etlidbe Morgen W̧alcung fällen, um

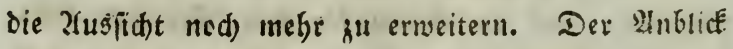

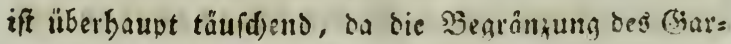
tens burdh eine fanfte Erberbobung bebed ift, uns alle vorliegende felder als zum Giarten geborig er= fdeinen.

Dies ift rev erffe Etantpunt, auf tem die the

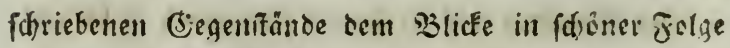
erfcheinen. (sieben wir jeşt ourch bie prád)tige Sins Dent aluee rechts bis zum Mittelpuntete res einen volls fommenen Birfer bilbenden (jartens; bier dringt sas

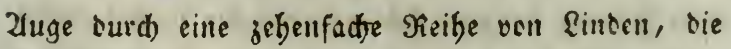
ven ber Seite betradjet, alళ ein ven ser Natur fien) and regellos erzelagter 2 salo fid barftellen, und ben 
25snberer um fo mebr überrafden, went er bey bem

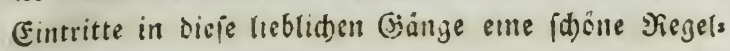
mẩigléeit entoectit.

2luf betoen Eeiten des Gartens erblift man in

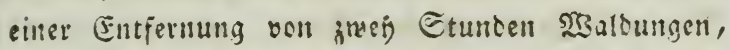
surd) welde alleen gebauen, und auf ten swifiten liegenten Feloern Gruppen von Báumen jo tăufdent allgewflangt fino, soj man sieje Saumgänge für un: unterEred)en fortgefert băt ; renn aud) bier ift feine

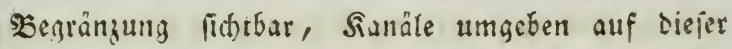
Eeite Den Garten. So wubte Das Genie bes $\mathrm{kr}$ ften Fffanjers affe siefe llmgebungen ju benthken, und dem Garten baburd) eine f(d)einfare Jusbebumg ju geben, sie surd) (jröß̧e uno Sdönfeit bas (se= müth eines jesen füblenden anfpridt.

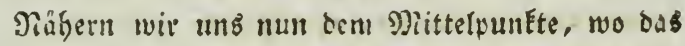
(5rop̃e, bereits wen befitriebene Baffin fid) befinter. Şier feffeln vier Urnen c c c von Earrarijaem Mia: mor die 2lufmetEfamkeit; geru verweilt man vor bie=

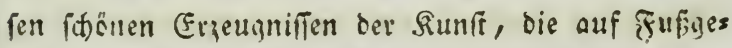
fellen oen inlänoifitem Marmor fteben; Sinber mit

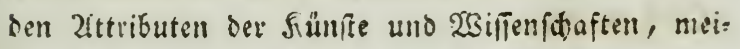
fierbaft in balberbabener 2lrbeit targeftellt, erböben

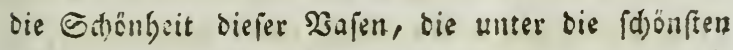
গ্জুerke cer Sumft gefören.

EGemals waren auf allen Eciten 'oiejes mittlern Gartens in ben vier Berticfungen niedrin gertnittane und an RattermetE gebuntene Błumengefräude; a!k 


\section{$-24$}

aher ber reinere (Befdimad biefe freifen formen ver: brangte, beletisgte man burd) folchen unnatưrlidten 3nang bie bolce Natur nid)t länger; fien wadtien sun biefe lteblichen (Beffräuche; ungebindert haud)en Stla und Sasminen thre Duffe, und ftrellen ifhre

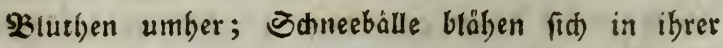

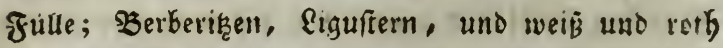
blübender $23 e i s c o r n$ entfalten ifre retde Wradt, und

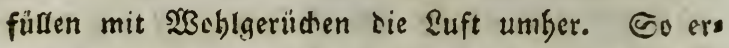
foheint un bier nun ber frrüblung in ieiner ganjent Sdönbeit ; ungäblige Madtigallen wählen siefe rei=

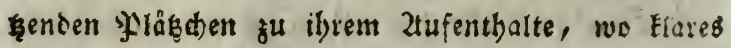
Wuffer in ber Näbe riunt, unb taufentoftimmig er. tönt ibr entjüutender Befang.

Gallen endrity biefe Bläthen $a b$, bann färigt tie majefrátifod)e Einte an, igre $\mathfrak{B l}$ lume zu entfalten, uno ibre Gernidse umber z̧u ftreuen. Uno fo at gntet man

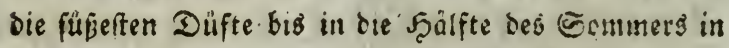
biefem obgfeid in veralteten Befdmadte angeieaten Theile bes Bartens; und biefe fhattenreiden alleen fino der eigentlide Epaziergang oer Jremben uno Einbeimifden.

Son hier aus fübret uns mun cin forrảge laufen= Ser Settentweg vedts ourd) einen Bogengang, zh bem Eleinen Bafin, in serfen Mitte die bolse (jas Yathee (U.) bem Babe entfreiget unt bic Saare augss wintet, uno an ber fidh ein Iriton hinanfd)miegt. 2ud) brefe Statue ift ven Eartarifich 2 Rarmor yon 


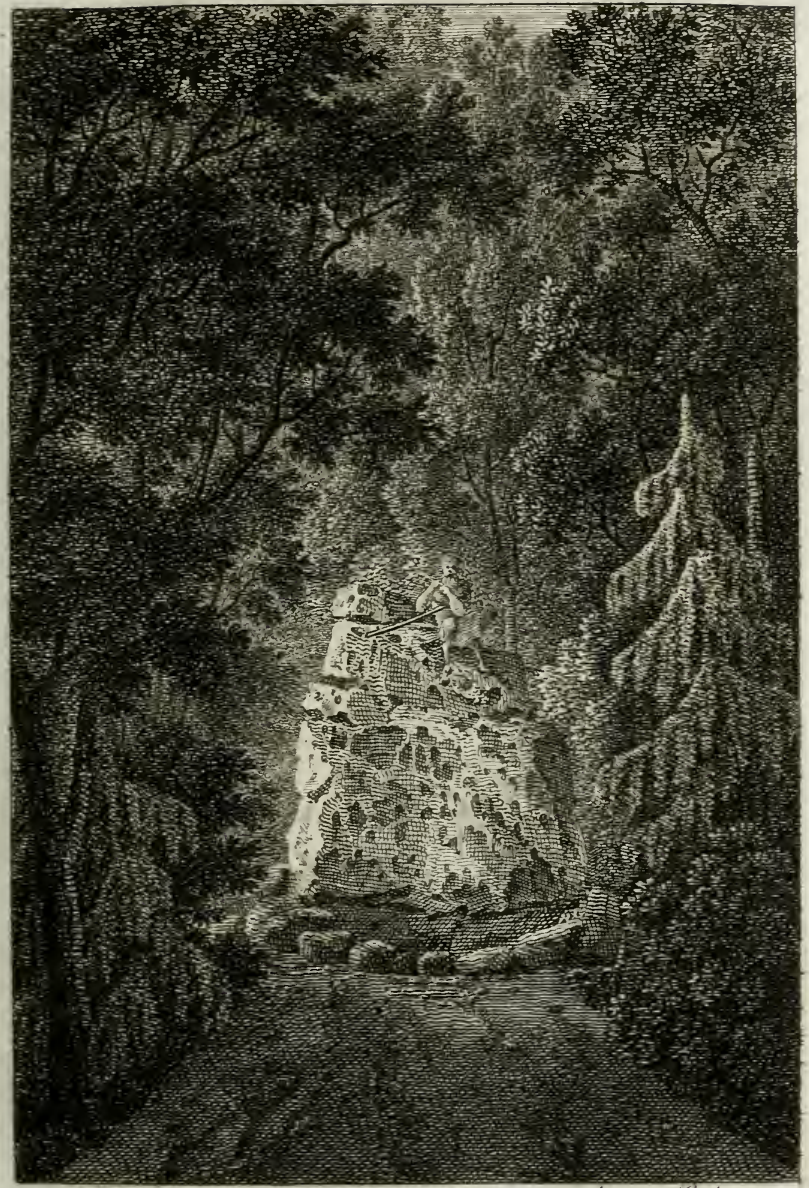





\section{- $25-$}

bem פittbauer Crepello, ber all bem Sope bes .Rurs

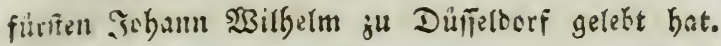
Dieie Bjalatbie if ein fotsenes Dentmafl der Sunte

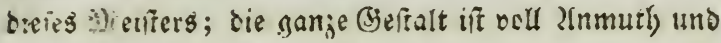

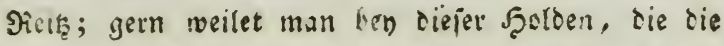
Edritte ses Soruberiehenoen feffert, uno in alts

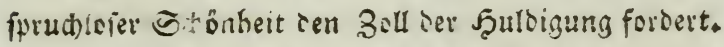
Innifrettig ift oiefe Statue bas Ealtharite Sunftiver in Dem Garten.

Şat man fĭ ron biefer 3auterinn loşzeriffen, und ben gefrummen $\mathfrak{W B g}_{\text {eg }}$ linEs eingeid)lagen, fo fommt man in eire Illee von ftoljen Sictftannen, in seren Seintergiunte, im fohuserltchen DunEel man ben Felfen Jुang ( T) erblicft. Der Butt liģt auf făon aufgetbürmten Sufíteiren, fwiclent auf ber nenn=

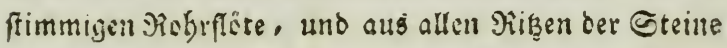

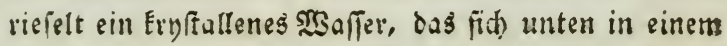
Eleinen Secfen fammelt. Die Statủe ift aus Sand: freine van cem jeşt in Münóen lebenten $\mathfrak{B}$ ildbauer

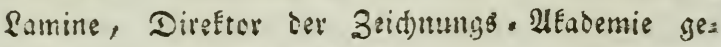
Gauelr, uno ie mefr man fie bctrachtet, befto inniger bedauert man, Daß̃ uns ticer Situfter ift entrifien werben. Sainter sem Felfen freigen glänjente Giltera farpeln uno Sirfen, vermijht mit SRadelbäljern

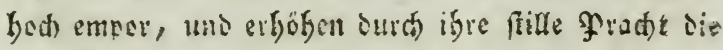

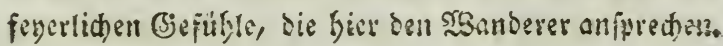

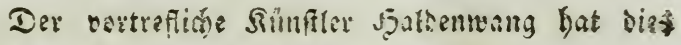

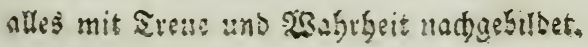




\section{$-26-$}

Midft weit ven lier ift ein Baffin (d) worin gruey (Srappen von Sintem auf rafierfisenenden Dras den frzzen. 'Eie finto ven Bley, breniit uno ehens falls aus cer Serlatienid)aft des Siöniges Stanstaus. Şier murmelt ein fïh) fohlänge(noes Bäd)lein, wels deß̉ bieber geleitet nurbe, um ben siögell jum ErimEen uno Raden zu dienen, unb diejen liebliden Drt ju erfrifonen.

Unendlid) Eonnten tiefe ganze 2fulage nod) ver: id)enert werben, werin bier unb oa Bäume ausges bauen uno ble freife, ermibente Siejelmäpigfeit ber ख્ge gefiont würten.

2fuf ber Seite bes Saffing fegt ein Bacd)us ( $t$ ), Der unferer zfufmerEfamkeit nid)t entgeben darf. Er

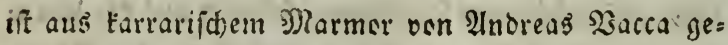
bauen, und dab Bsegenffüc $i$ einer 2lgrippina, auf

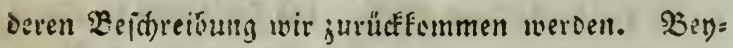
De framien ehedem am Fingange Des \$iannfeimer (5)lopigartens, und wurden in Sabre 1768 nach

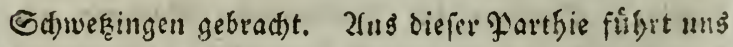
ein (d) rảge laufenber $23 \mathfrak{B}$ queer burd) eine prädttige 2uthee von Sebfaftanien; wir treten aber glẹid ber. aus, und feigen eine Sretpe, wolauf zmey :öwen onn Sandfteire ruten, binunter, nach cinigen $\subseteq$ doit=

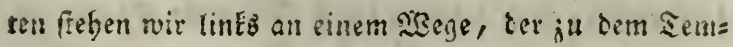

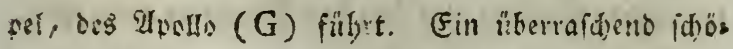

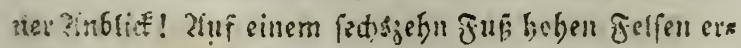

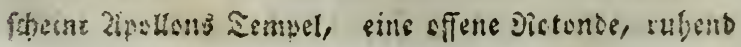




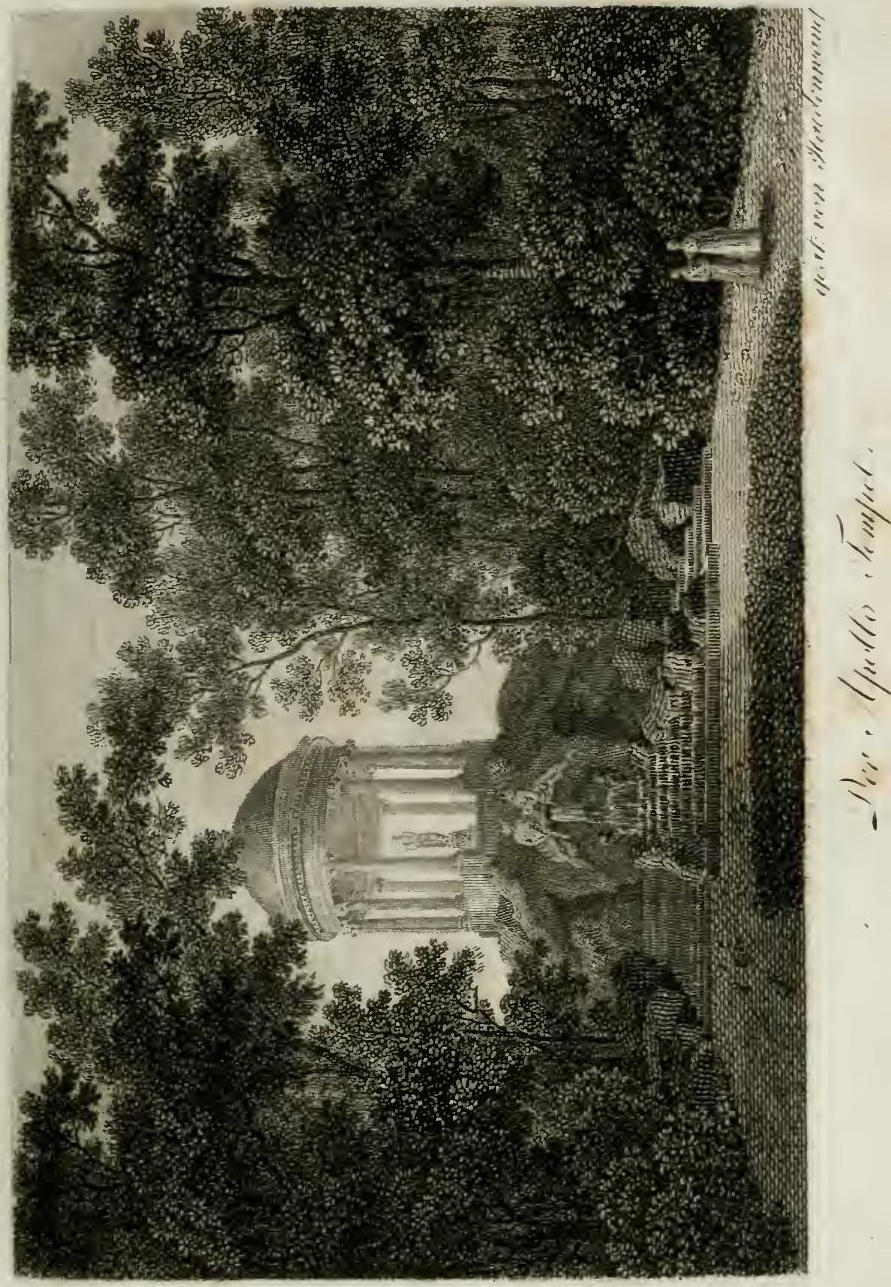



auf axt jonifaren Saulen; auf einem \&ufgeffelle von

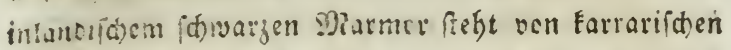
Marmat ven Dem Mitter wen Sierfichaffelt Der Giott mit der Snra. 2tm Fuje des Sempels ruben auf dem Giruncfeifen ;wen Siajacen ron demfelben Rünfter unt gieşen als iffer Urne einen @irm bes belleften

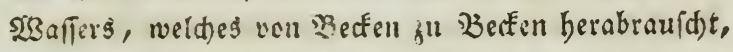

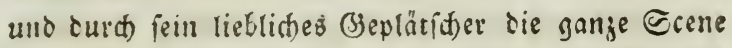
belebt. Unter cem serrpel fino in ten Felfen eine (5ivette, uno veridfiedene unterirroifde Bange, die

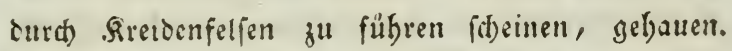
Iritt man auf der binteren Seite des Sempels ber: ars. Fo fintet man zroen Serrafien, bie mit Gitters werk von (Eifen, weldbes vertreflid) gearbeitet ifr, eingefaft jint.

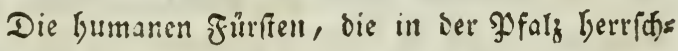
ten, veriagten den fremben der Sïnfte und der fäönen Siatur nic, fid bier zu einem freundidoftli=

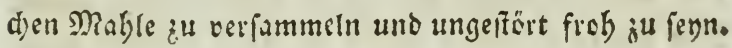

2(ud) biefen Sempel mit feinen Umgebungen bat Saldenwang mit Siumft uno Biefühle, Dargeftellt.

Eteigt man auf ber 2sorberfeite rediter J̧ano die aus rauken gelsiftüfen beftebente Ireppe binab, fo

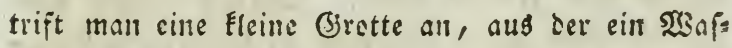
jerquell hervorffrucelt, uns worauf ein withes So bein

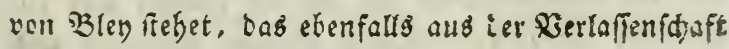
Des Sönigez Etanis̆taus Enmmt. 
Der Borplats bes Sempels war uriprůnglid nad damabliger Sitte, wo mall in iedem Barten ein Sbcater baben wollte, wirklich für thentraliẹche $\mathfrak{B o r}=$ feflungen angepflanjt. Lattenwerk uno ciferne Bit=

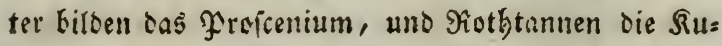
difen, Einige Etufent tiefer ift im Salbjirfel ber

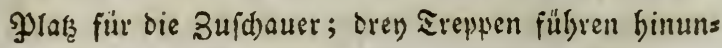
ter, und auf jeber liegen zwey Eoleffale Ephynte. Die Bñume wurben gewiflenbaft unter ber Edeere

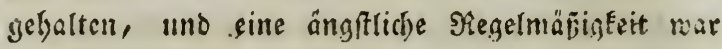
allentbalben (id)tbar. Doch als man endlid) aufgërte, fiid) feruer an ber Natur zu veriündigen, lię̧ man siefe Sannen frey emper mad) fen, bie nun in foljer

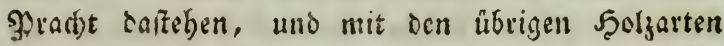

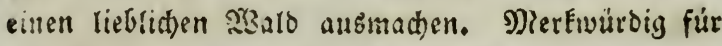
Sen Forftmann fint auf biejer Stelle bie Ulmen uno SIEazien, weldhe in einem 3eitraume ven pect) 8 uno

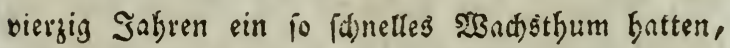
Daß mebrete baven über zwey Slafter feat gaben. Die Lltmen (Ulmus sativa du Roi) inteffen wad)s

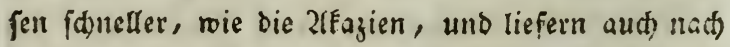

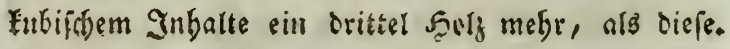
Werjđiptene biefer lumen fino über neunjig Juß

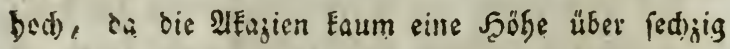

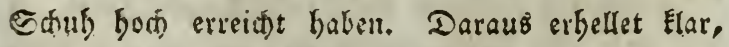
Daj ken gleich) gutem Eroreiche sie lltme in forftlider

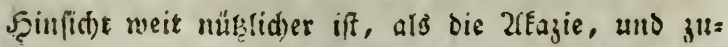

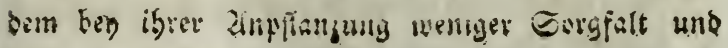
Sultur erfordert. 
Iim Sagre 1803 ben gten Ceptember ward in

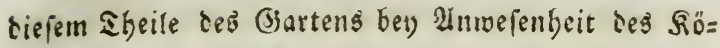
niges und Der Röniginn von Sdweven $216 \mathrm{cnt}$ s um

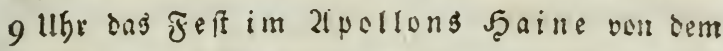

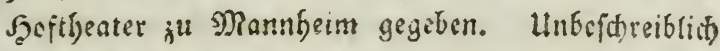

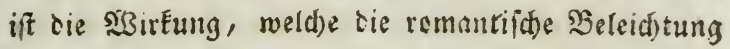
machte, bie ser damahlige Bartentirefter SFeer ans gecrinet batte. Nirgents warb man eine Flamme gewabr, und sod) war überall eine slarbeit, welde die Eleinften Biegentiante erEennen lię; in sem Ery= frallenen Suelle, bei aus cer Utme des গajaden iith ergief́t, brad)en fid sie êtrablen ses fanften Sodims mers, uno fprithten umber als leuctence funEen;

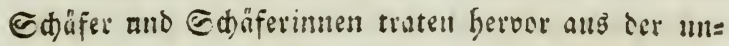

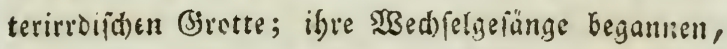
begleitet von Flöten und andern Snftrumenten, die, verbargen in sen Geitengebüithen Gerüberfiäpelten; uno fo machte, begünifigt ven dem vemantifiten $\varepsilon_{0}$

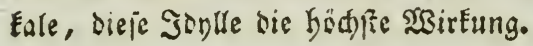

Fa sffitet fich zwar, fteht man in siefem Sempel, bem 2luge Eeine weite 2tusjictit; Ded) fiebt malt ven cer yordern Seite auf bie Srangerie, und ven ier Serraffe erblicft man jenfeitz des fianales die engli= fthen żnlagen. Dieje ganze Parthic bat einen ro: mantifd = feverliden Sarafter; fie labet zu ftillen Ses tradtungen ein, und ungers tritt man ans diefen fhonen Uimgebungen Geraus, seten Finorut nut Durch) einige angebract)te Begengünge uns Şecten, if 


\section{- $30-$}

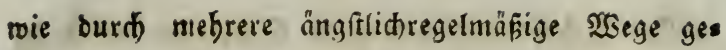
ftort wird. Dies iu verbeliern ift aber meser eine forwere zlufgabe, nod) würde es beträd)tlid)en 2luf» mant verurjact)en.

Sey beitern Früglings: oder Gommertagen ift biet

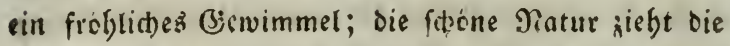
Nienichen biefer, unt nun fiebt man fier einen mun= tern $23 e r e i n$ ben einem frugalen 9 iable; bort im Bjes

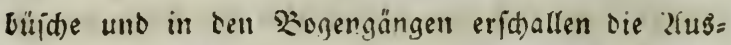

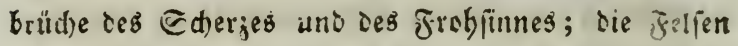
uno Brotten fino belebt, und eine unbefangente Şei= terfeit erböbt sie Bergnügungen, welche Natur uno

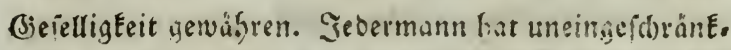
te Frengeit su thun, was er will, fo lange et lid)

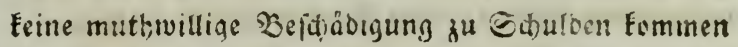

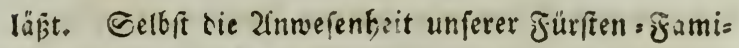
rie legt 9iiemand einen 3mang auf.

Serlafien wir nun zpollons Şaus uns wenten

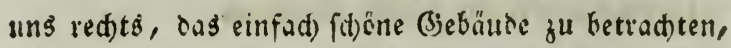
oas hier vor uns leegt. Es if das babbaus $(H)$. Der Dberdiretter ven sgigage bat den glan baju ent= worfen, und bie cole Ausfübrung madben feinem Beifie Éfre.

2im Eingange begrünen unz 21mor unt ein Faun, fän gearbeitet von dem verforbenen Şefbilobauer Sinct. Siun tritt nan in einen länglid,t runsen Saul, an befien Secte alurora eridsemt, wie iie sie

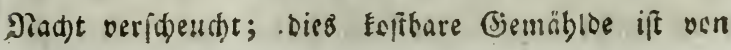


rem nürtembergiften Şefmahler (juibal. Bwifhen ren rier Sbüren freben in Slijithen vier vergelcete

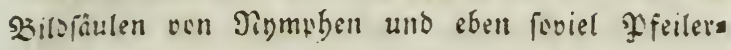
Sifde auf geicenen (jireifen. 2fuf beyten Geiten die: fes lisblicten Eleinen Eaaleg befinden (ït) trey Sias

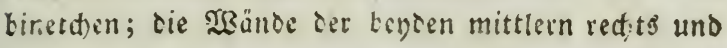
linţs find von Gipssmarmor, umgeben ron Eruct= 2liteit; in iem jweyten if eine Eeftbare Sapete von direlifhem Fapiere, ein Ramm oon Mutrmot, uno cin Sirmlendter aus ber ebcmabligen Fyergellän = Fas brik ju Frankenthal, umwunien mit ăup̈eren fönent Funitichen Şlumen, Die ein Frauenzinmer verfers tijet but.

Im tritten Sabinete if ein 2lfkwen, sen jwey Eanelitte Căulen ran alabafter jieren, uno jwifien zowen prädtigen Epiegeln ftebt eill Piubebett ron Eutbarem Etcife. Das Betăfel if̂t von austanto fdem, befonders von refes: = und Sontijetgolje; in cen frullungen find oertiefide Sandichafien ren bent veremigten Sabinets̆mabler, Ferdinand Setell. Iluf

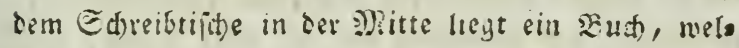
der dre Sameil ter fremoen ent?ält, Ivelda die

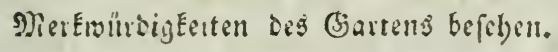

Das mittlere Sabinet auf ber antern Eeite ift ebenfalls mit Marmur uno Cturf = 2rbett rerjiert;

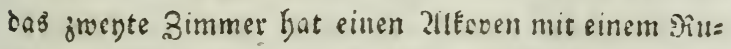
bebette, Seflen lleberjug forobl, ais oie Eapeten uen rotbfarbigem sleEing mit letendigen Blumen fint. 


\section{$-32-$}

In ber britten Slbtbeilung ift nun bas $B a b$. Der Beden, die Sreppe und dus wab felbit fint ven inlänsiidem Miarmer. Dben Dranirt fit) ein weifjer

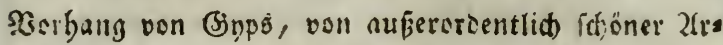

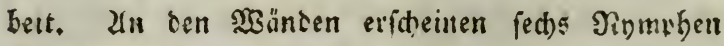
mit ibren Srügen, balb erbaben geartertet; auth

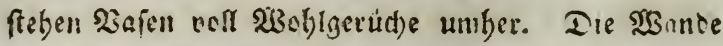
fino birottenartig mit Sirvitall, 2(methyit uno ancern (E);engunten aus ben edlern Eteintetche geziert; man fieft Ffeiler ven beutjden 2llabafter uns sisänce mit virninifatem Eumad) angeiegt. Die Decte it in rets fitirdene fester eingetbeilt, tII deren Füllungen Epteget angebrad)t jint. 2(uz einer vergotoetent dine

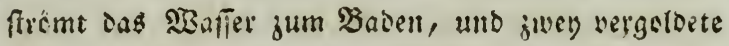
Edylangen fFeyen nad) Der Willfithr des cäabenten warmes oder Ealtes sisaffer. Siabe bey tem Babs bauje fielyen jwey (Jiruppen von Findern, init Biegen uns Sodten (S), vant tem Sefbildgauer Sinct.

Berlatien wir nun siejen Iieblicien Zlufentbalt,

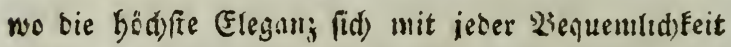
verbinbet, unt betrad)ten wir sie neue ecene, die fid) unfern Btiffen offinet. Ein bunkler (jang liegt vor uns, unt in weiter Entfernung exfdeint eine freunstides Sandidfoft (I), breitet fith nor uns aus, bie fo täuideno nad) einer Zeid)nung oes Safmabs= lers ferounano fiobell von einem gemeinen Suncher in Mianngeim, Samens Intefenmülter, auf eine

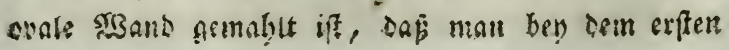




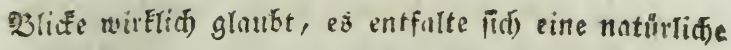

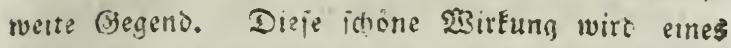

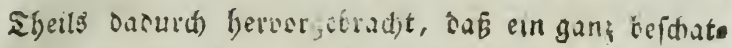

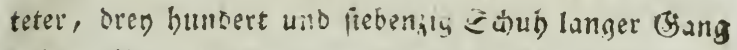
Dabin fubrt, theits bap sie ?andihaft auf ein Ooar gemahlt ift, uno oa mon nabe var oer Mablerey an einer Elemen Ourd) einen Ëlnffluben Fetien gebreche= wen Cirotte heht, in seren Becten dat ban rer Dacfe

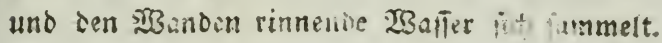

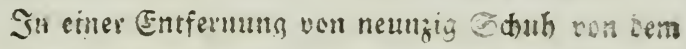
Basbaule fommt mau zu bem fossenunten s:ogets Raffin $(r)$. Iites if ein runder, mit अitierwetE

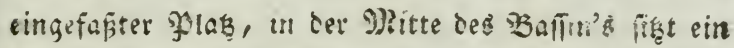
Uhu in zorniger Ctellung, uns phent $23 a$ fifer gegen die übrigen grö̧ern uno Eleinern şögel, die ober

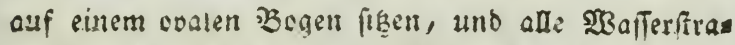
len auf den Raubrogel berabipeners. 2uf benders Ceiten fino vier sogethäuier uno imey, niebliche Sas fiettetchen, ausgegiert mit bronzirten bas-reliefs, farbigem Gilap̉e, 21фaten, 2(metbyften und anvern etlern Gteinen.

Der neuere und reinere (5ejhmact vernitft twar forche 2nlagen; und ein natürlider 2 Bafferfal mistce eine unenolid fobonere șirtung machen, als̆ ive

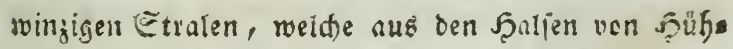
nern, Ffiauen, Srutbübnern uno andern klemer sös geln berabitromen; indelien gefält dod oteje peffier. lidge Ecene ned vielen; und bie Lebertaja)ung iff of 
febre grof, wenn man fremse bieber bringt, und ets

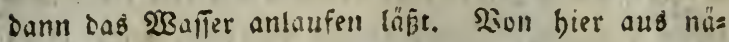
bert fich man nun ourd einen duttern Giang, des sben bereits beidhretenen Ferfattue. Fin Eleines Sorgebaute ideint an hen frelien angebaut in fegn, uno inau Eann fich Dem Cimäblice nur auf fünfjelyn Eaub nuben.

2lus diefem (3jebåute tritt man burd) eine SGüre linss, uno befindet fich in einer Saptanien=2llee, welde gleich) einem (sürtel den ganzen, nach alt s franzifitichen Etyle angelegten (Batten umgibt. Diefe Bitume find alle prödtig gewachien und wëllen lith ju einem grünen Edirmbable gegen die Gilut ber Gonne, und ber sicht neben an flefiende Santal ex - frifhet biefe S(battengănge ned) mefr.

Sin einiger Entfernung redhts füfrt eine Brücte über einen Fleimen 2 seifer, ben man ganz üEerfiebt,

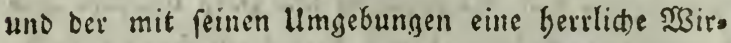
fung macht. Traucimeiden bebecten theilneife da Ufer, uno igre Gängenden Zweige fpielen in Der $\mathrm{kla}$ ren Fluth; (joruspell von Eamadifhen Mappeln uno Ellern erbiben bas Mablerifite bes Eangen.

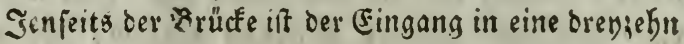
Miorgen graß̧e Baumidule $(X)$, welde über 240000

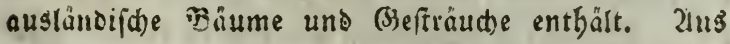
biefem reiden sorrathe werten alle berridjaftlide Gärten in ber $\mathfrak{P} f a l_{3}$ angelegt uno erbalten, und aud 


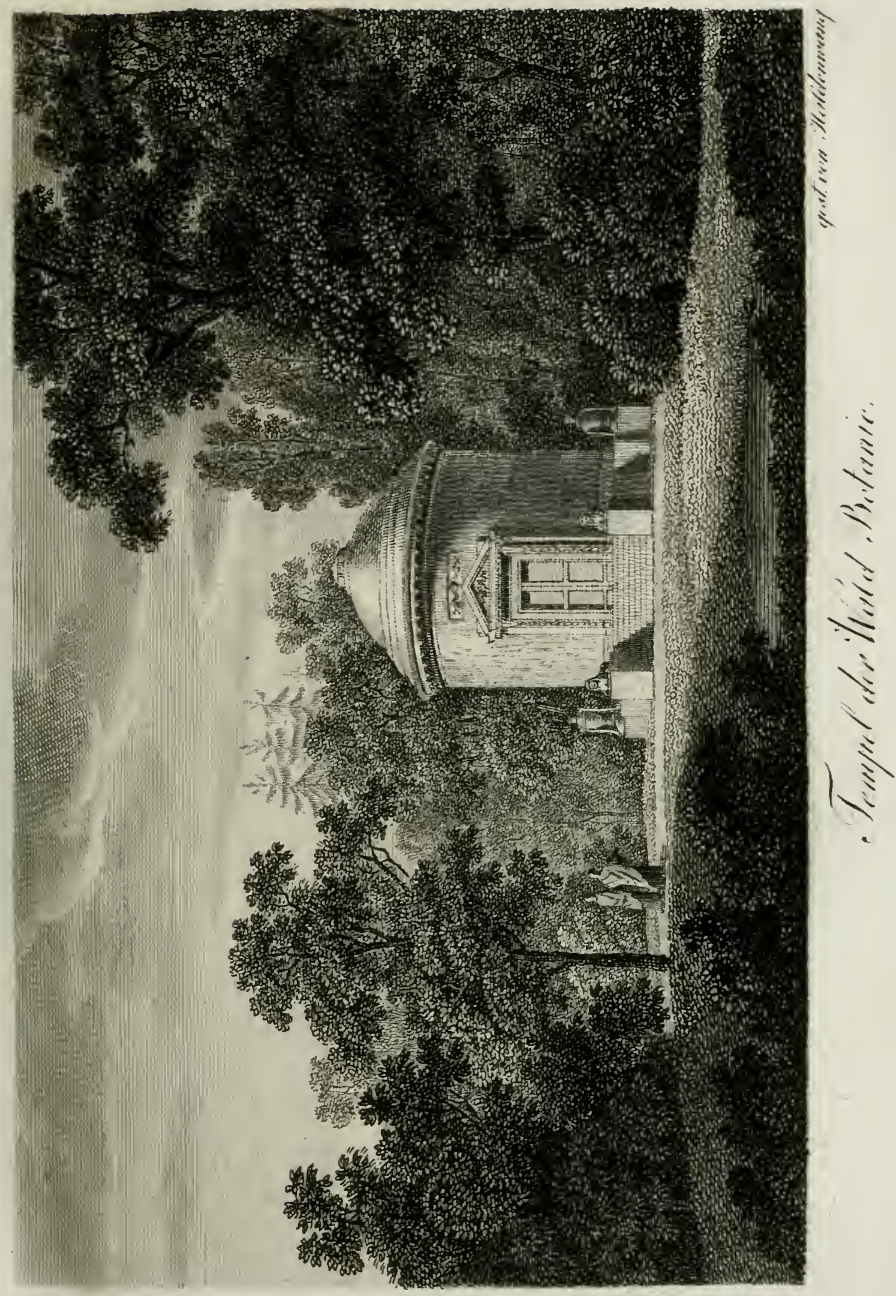





\section{- 35}

Der Riekbabe: Eann bier Eăufich befommen, was er zน feinen gifanziungen bedarf *)

Der צrseg rechts fübrt am Sande dieles romanti.

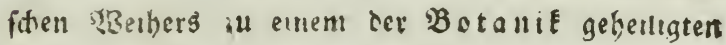
Sempel ( $K)$; vor Defín Eingange auf oer Ireppe

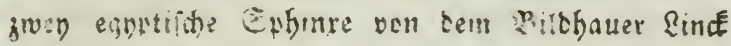
liegen. Er uft runs, oen aehaumen Steinen aufges fübrt, uns bat oie Jnichrift:

\section{Botanicae Silvestri}

\section{An. MDCCLAX!II!}

Die fich auf cie sáme und (jieltr. dhe ber naber Pflanzung bialeht. In dem Sempal, weldier Das gicht cura cine Seffnung in ver Siuvel erbält, ftebt cine alecucrifhe Statue von tarrarifonen Marmor, bie aus Stalien gefornmen it: es lit eine weiblidge Figur mut einer soulle in der fand, worauf man bie

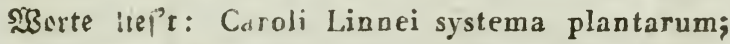
ju ibrent Fufien fteft eine Bafe mit Gewâch jen. Zwen Esifbare Saien oon Marmor mit allegorifden 2ierjlerunger, erbicft man in פriichen; uno über vier dltären mit B!lumen, frrúchten uno Gjartens merfisugen bängen In Medaillens die Biloniffe ven Sinné, Scumefort, Flintuş uno Ibeopbraft; böber sben fino die vier 'jubresizeiten in bulberbabener 2fis

*) Das vorffinoige Sergeidinif biefer Syblarten, fo wie jenes Der fimmtlichets Mflargen uno Dufarten,

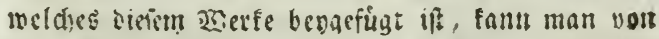
Dem Gartenoirettor Seiber mit ben bevgefęrent. Preifen befommen. 


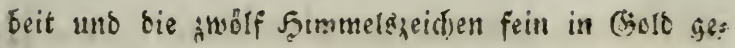

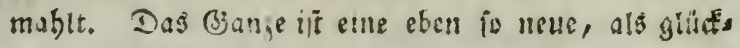
lid)e Erfintoutg.

SBar fino nun in bem fogenannten englishen Gar:

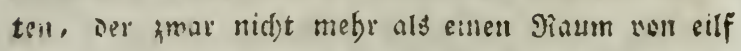
STarnen ennimmt; aber bennech bie therraidentos fren 2abrechielungen barbietet. Nabe bey rem Tem= fel ift stsalfer, weldies aub bem oben genannten

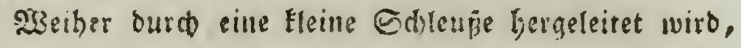
une ourd) das mumelnte Jian (d)en feines jalles dir

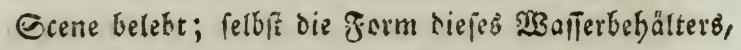
einige phritcen, Das fäone șsiefenthäld)en, uno bie glüctilthe Bufammenftellung ber sfifangen bringen bie

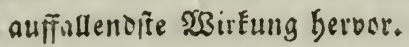

2lrs oiejer Ibeil des Bartens angelegt warb, fanb man bas (sertipe eines Beiterz uno feines Fferbes. In ber red)ten Sauno hielt el ein bier $6 d u b$ langes Shroert, uno in dev rinfen sin Stüt Eifen, dab

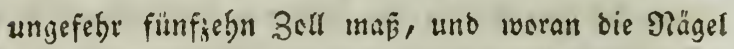
nod) waren, manit es an einen Schilo befeftigt war, weven man aud ienen aus Mefing verfertigten Sheit

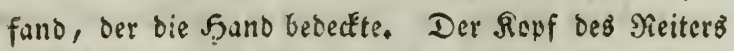
lag fechs bis fieven 5 d) Diumpie, man fanto übrigens aud) feine gropen Epos ren, einen howpaum und cine Stange, beren vicrs

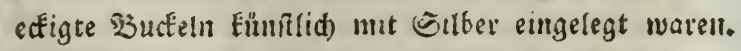
Mrfpringlid) war biefer Sheil oes (siartens zu einem 2rob cretum veftimmt; Darum fincet man ausb biet 
Die feltenffen Bäume: Juglans nigra (fowwarge ames riEanifde Nüife) Betula alnus laciniata; Gleditsia triacanthos; biejer fhone Baum bat fectzebn 3ed

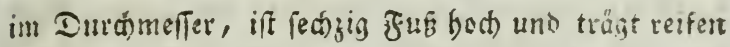
Gamen; Gleditsia inermis if beynabe eben io fturE, trug abev ned) Eeinen Camen. Gleditsia monos-

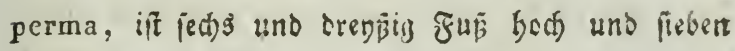
3atl bick. -

\section{Tulịanenbăume (Liriodendron tulipifera)} Pinis Strobus, P. Canarlensis, P. Mariana alba et rubra, Carpinus virginiana, Acer Negundo, Acer striatum, A. tataricum, Cercis canadensis, fraxinus pubescens ctc. fino alle feffr fiark, uno Eringen Eamen. Die größte MerEwüroigkeit aber if ein Cingko biloba, oder nad) Dem neuern Namen Salisburia adianthifolia. Diejer bier zu Sance fel= tene \$aun bat zelen Boll im Durdmeffer, und eine

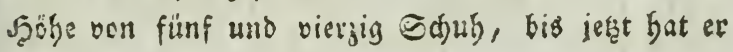
aber nod nidet geblübet.

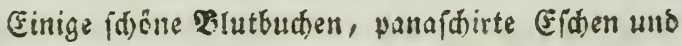

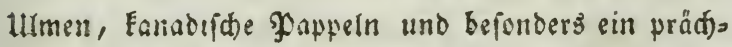
tiger Baum, Populus laevigata, erregen burd ifreen Eübnen und frolzen \$3นdjs das Eritaunen einez jedent.

Tiefe ganje 3intage, wo frembe uno Embeimis

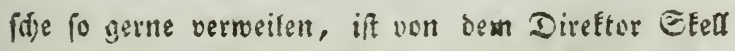
gleich) nadi) feiner BurüfEunft aus Englans mit vielem (Befd)mace angelegt rooren. Der Ciartentunifizer

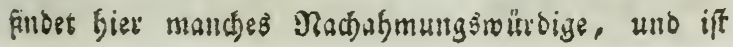




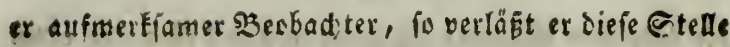
nidt, chne fid mit neuen Kenntniffen beretdert ju haben. Jeget Eehren wir mieter gegen oen Sempet

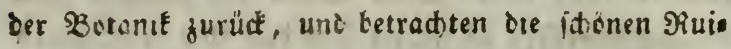
sen $(M)$, vire gans sabe an biefem Iempel frebell, - bye ourch eine Serpflanjung abgefondert zk fegn. Cie llegen an bem Ende bes̉ Biarteng, deffen Begrän= jung lie hier ausmaden. Maḩlerifd) von Dufifeein erbaut, fabeinen fie die Ueberbleibfel einer rémilichen

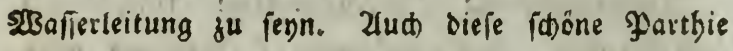

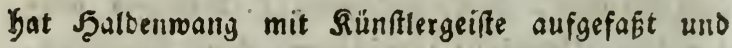
Dargeffellt. Son oben raufidt ein Elares, reines SBrunnenmaffer herab, weldhes vori der Mafdine $\mathrm{N}$ bergeleitet wirb, und fowobl den $23 a f f e r f a l l$ an bem Iempel atwellons bildet, als aud bey dem Sogelbafiin ftrömt. Ded bier in Diefen verwitterterten Mauern, über Die Der Sturm ber Zeit bergefabren ju jegn fdeint, ift bie 23 trfung des fallenden $\mathfrak{B a f f e r}^{2}$ übers rafdent fidin, uno belebt bieje Ecene, wo nirgenos

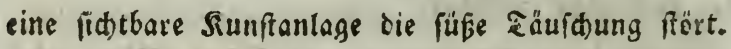
Sdeinbar regerlos, wie bie Natur idiofft, erficheinet hier bie remantifde Fflanzung, Die frey uno üppig emporfteigt, und man bedauert nur, dẩ ber beid)ränE=

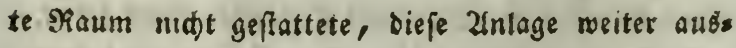
zubegnell.

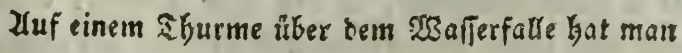
eine weite 2(usfidst in bie unliegente Eandidjaft.

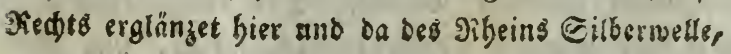




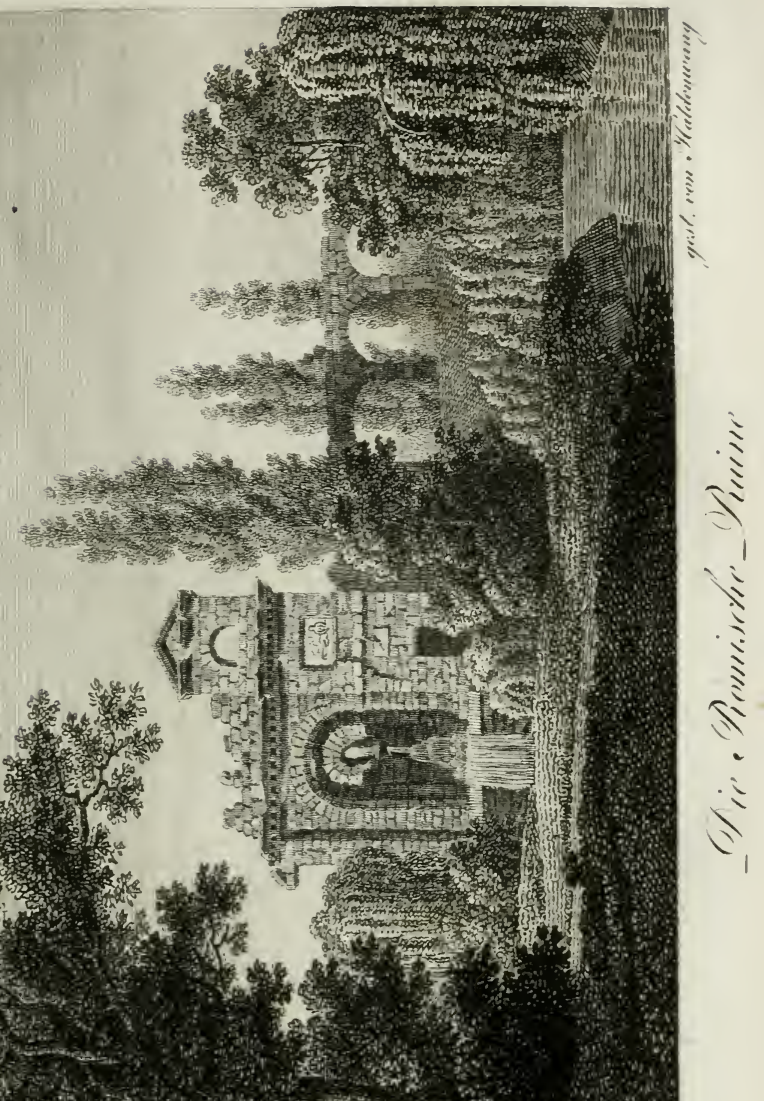

An की

The

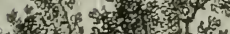

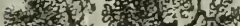

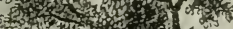

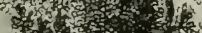

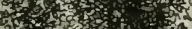

Hot Ty

1.t.

oxis

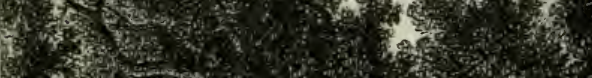

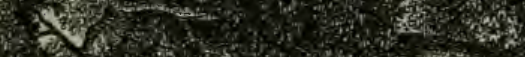
(3.)

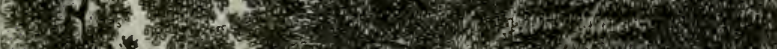

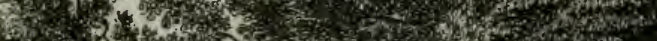

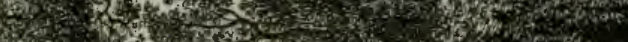





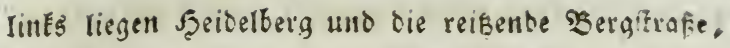
uno vermäts befnen fich oie paradiepichen (Sefilie aus,

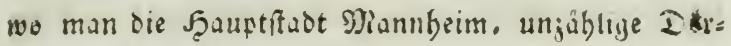
fer uno in Şantergrunde ftolje Gebirge in blauen Dufte erblicft.

Ned) ift bier ein Eleiner Mreinberg angelegt, in sefाen Mitte ein fünfig Eduh boher DbeligE ftebet. Sisn bier aus fübrt eine Eleine Bruffe tiber den Sas

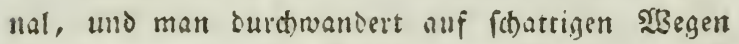
Diefe anlage, wo alle Bâme uno Gtauden vortrefs

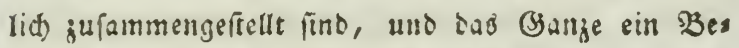
weis bes reinften Sieftomactes ift.

Sest betreten wir einen Sijeil res Gartens, wo in Iabre 1763 eine Dienagerie angelegt war; mef: rere Gattungen van Scübnern, Sauben, Euten,

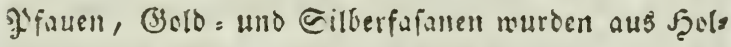
lunb verjorieben, allein im Sabre 1778 warb bie Nenagerie mit ber Fafanerie ju Sandbaufen ver. einigt, uno biejer Fुlas in SPflanjungen beftimmt.

(j)eid) bey Dem Eingunge if Die Nutterftule $(Y)$, wo alle iene f̧olgatten, bie curch) 2lbleger forte gepflanjt werben mülien, lid) bifinden. Saieber ge= bören die (sefdichter Betula, Coryllus, Rhamus, Rubus, Ribes, Clematis, Dirca, Viburnum, গio= fen uno andere mebr. Şier erbebt fid ftoly bis ju ciner Şäbe ven 54 Sdub eine Magnolia acuminata il der Pract ifper Silberblüthen, fie bat irey Sd) drey 3ou im Umfange, unb trägt jwat nur fparfam 


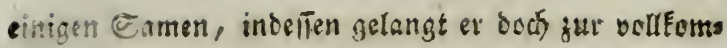
menen Netipe. Der heuner benundert ferner eine

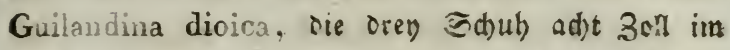

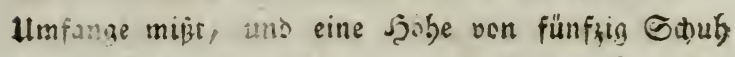
erretht bat, Sie blitht; rouk alle Gabre, feat aber

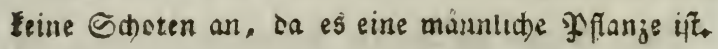

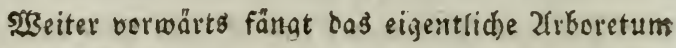
(X) ant. Errt vor vier Jabren ift es auf Befegl Sr. Sönigliden Sobeit, des Grosberjoges, anselegt

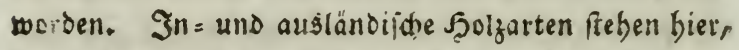
foviel es mëglid war, beyjanmen, und die im Satas. loge mit Arboretum bezeid)neten Fflanjen zeigen dert

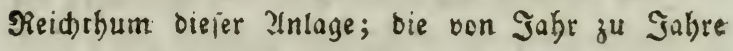
vermegrt wirt. Da Se. Sönigl. Şobeit, der Gircos= beriog, felbit nidt gemeine Genntnifie in diefem fors d)e ber Naturnififenidgaft belifist, fo find bereits be Deutende Summen verwendet worden.

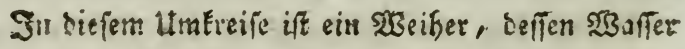
eine lieblid) angewflanzle Sonfel umidliesen, in beffert

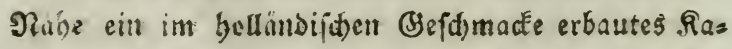
binetden fid befindet. 2fujerhalb oiefeż Şäuddent

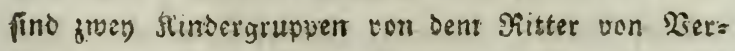

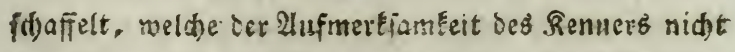
entgefen durfers. So vereinigt fith aflenthalten it

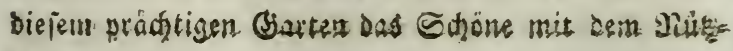
riderers 


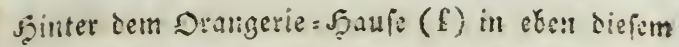
Zlberetum firben in ien Esmmermensten die Giags

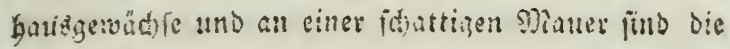
atpenpfanjen in freyen Ronse angeflanjt.

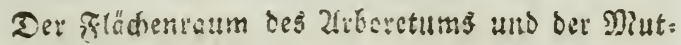
terfidule en Gäte feds Mergen.

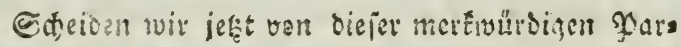
thie des Gartens, und tieten wir sbatbalb Geraus. 9iectit breitat fich baun bor bem Bitie ocr fitsone

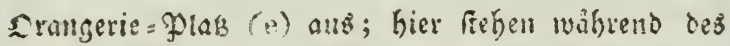
Gommets fechs gundert und or nspin Drangen, forser, Binnaten, Migrtben und andere Bäume. Die mit (f) Bezeidneten Sierecte fino zu einem botanilichen (jarten befinmt. Diefer ganze Plats it mit einem jiemlich breiten Fanale eingejä̈t, ocr joludg! in fain=

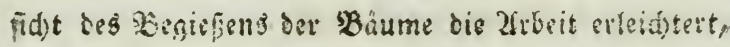

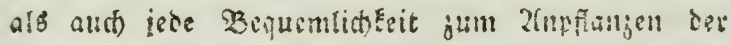
ભ gervảbrt.

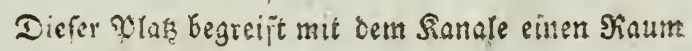
oon fïnf Morgen.

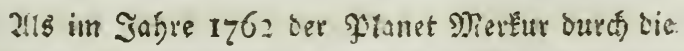
conne ging, lief der verewigte arl Sheodor an bies

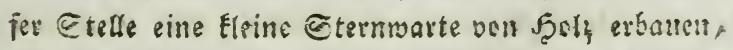
wo ber gelehrte Sefuit, Egriftian Shaver, oamals

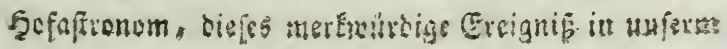


gूlantert = Eyfreme beobadtete. Im folgenten Jahre waro jotann anf iem Ediclie cine wirklide Eterns warte mit einem bewegliden Dache erbaut, uno mit Dell pergüzlidffen Sntrunenten bereichert, weldje ber

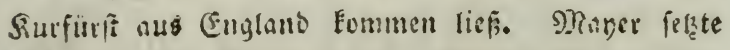

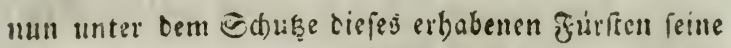
aftrencmifden zirbeiten fort, weldse bie gelefyrte gie= publik int all ber 2istrdigung aufnabin, oie fie vers sientert. Damals beftinmte Miayer bie Fुerbebe vou Sd)weţingen alif $49^{\circ} \cdot 23^{\prime} \cdot 4^{\prime \prime}$.

In ber Folge warb bie Mannlgeimer Eternwarte erbaut, bie eines jener unvergängh(c)en Denfmäbler ift, welde ser Sadjwelt tin Finfm Sinrt Ibectors

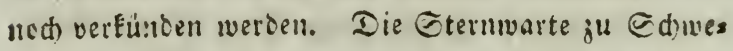
rimgen ging alio wieter ein, uno alle Snifrumente wuiden nad) Miannbeim gebractst.

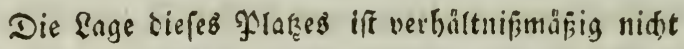
minder fdjön, als antre merfwüroige F̧arthien des

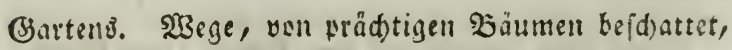
fdhlispen ifn sigen Cüten unt Diten ein; gegen S23eiten erbebt firs in einer üprigen Ff flanjung im fdauerlicten Dunfer ber Eempel 2(pellons, unto nöro=

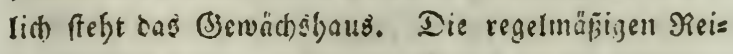
ben ber Drangen $=$ Citronen $=$ Sorber $=$ und Branat

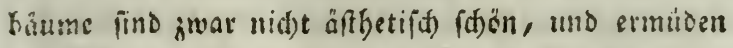
das ztuge; allein bafür werb man burds bie liebltd)= 


\section{$-43=$}

fren (jerủd)e entidäbigt, weld)e bie Suft umber eto futtlen; und ferffit das zuge finter Sdjöngriten; liz5. lich) blügt sie Citrone, unter bunfelm Cerün glüft die Golderange, und bie brennente Giranate frrable twujendfach auf oen 3weigen.

SerläBt man biefen Drt, fo tritt man in einen fidattigen (b)ang, Utberwsitb you eifermen Sieifen, ber fechjegn Godub bed und jwölf breit ift. 2fuf ocr rertern Seite if er mit witten Relien (Hedera

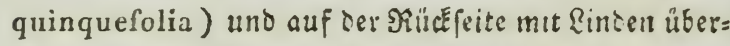

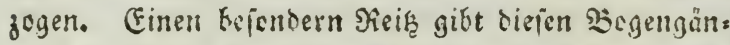

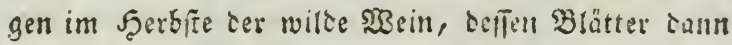

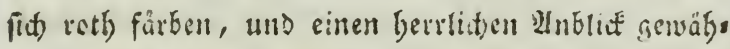
retr. Dieje grünen Geneilbe bifoen mit oem Epeife= fale B uno dem Diangeriegebăute $C$ oen grefen

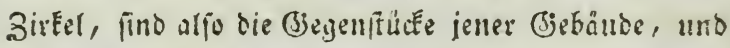
begränjen io den mittlern Garten. Dieje zinlage if gnar, wie bereits bemerft werten, nod) in alten Eryle gemadyt; inberlen bat man in neuen Borten, wo der Bjefdyact if verfenert rostoen, und wo man fid überfaupt ber פatur megr genäbert bat, alles getban, un bie Siuntraffe zu mildern, uno fo

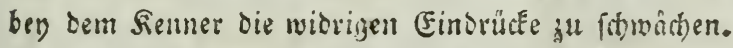
Uno wirllid) vergint man aud balo bas forblerbafte im Einjelnen, wenn man die prädtigen Saumgånge

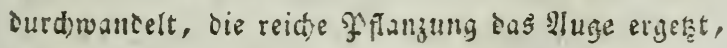
uno bie Sumitwerfe, welthe in den Baplins aupgeptent fino, die ztufmerffamerit auf lit) jiehen. 


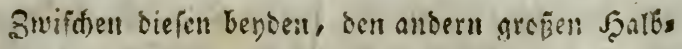
gitket brloenden Bogengängen, erblidét man ziwen

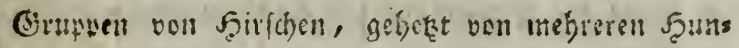

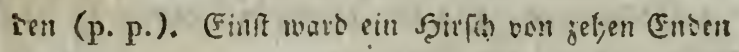
sejagt; ras geningfrete Eljier fioh, rettete fich in ben Baiten, mis warb ven ben satmien anf cheil biefem

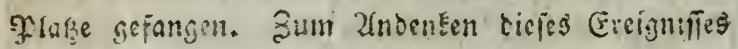

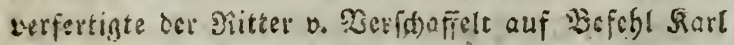

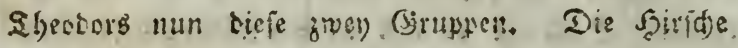

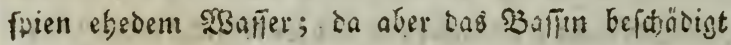
3vat, uno einell felst unangents men (Jjerud) verbreis tete, fo ward es jugeworfen, in cinen Siafenplas una

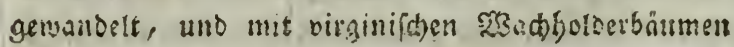

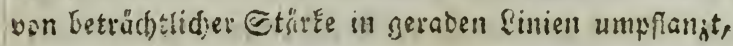

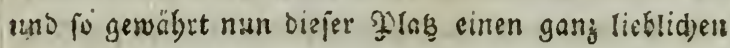
2Inbsict.

Deen und unten an diefen șachbotberbanumen befinden lich vier liegende Statüen, von Dem Mitter von Seridaffect alts gerbem Candfeitie gegauen, und fellfen bie vier Elemente vor (q.q.q.q.). Die Erbe erEentet man an ifrer Maucr frone uno on ocm Söwen; ein Triton mit einer lline und einer $\mathfrak{M}$ us fidel frridft fid als das Simkito bes Șafiers ant: 23 ulfan, mit ber phrngiften $M$ Müre anf bem Fowfe, unt mit feinest 2sttributen, bem Sammer uno

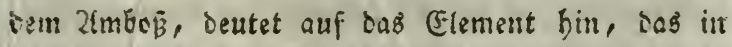

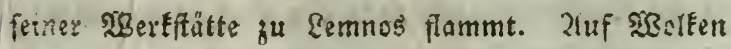

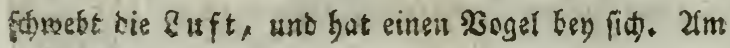




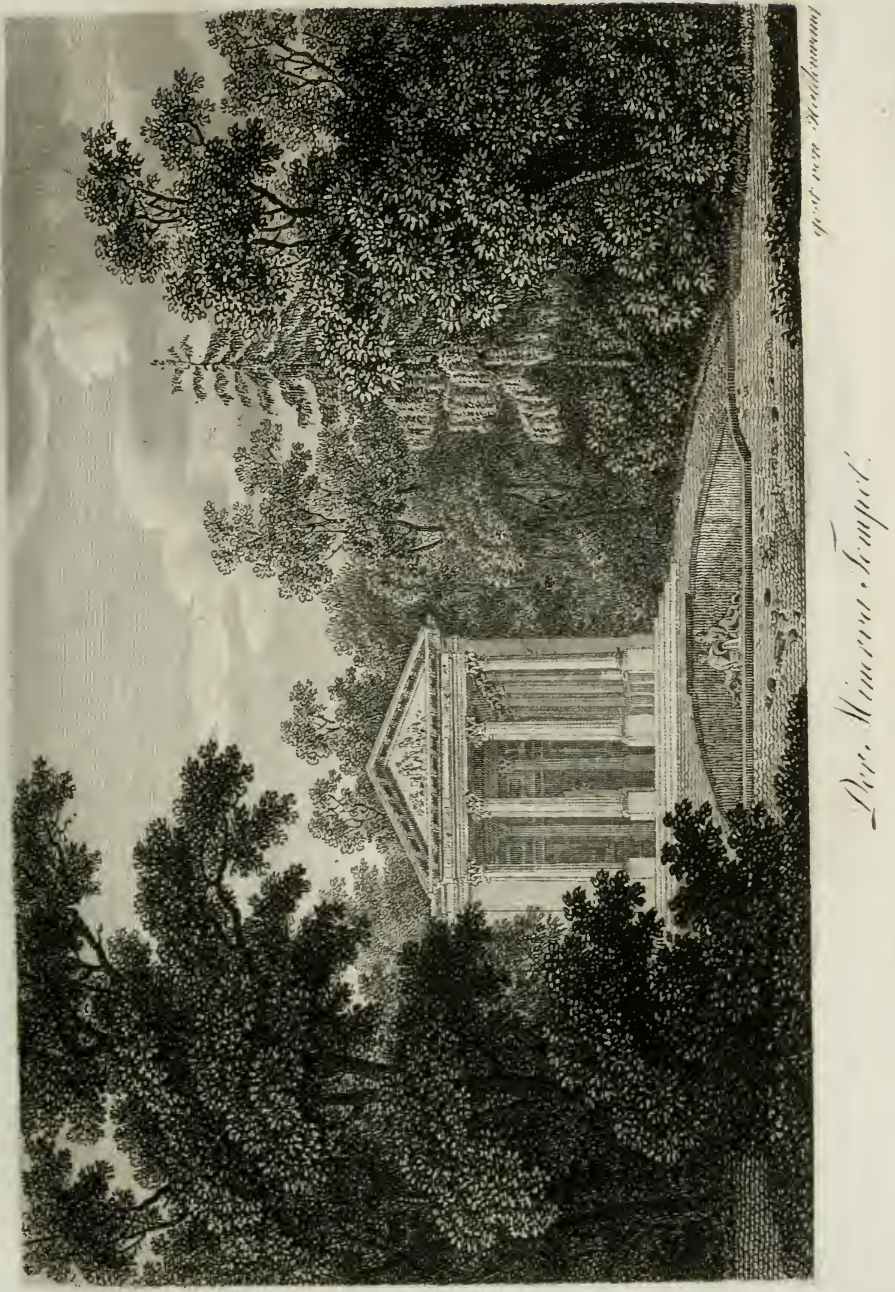





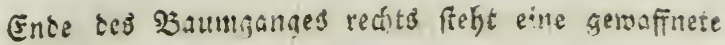

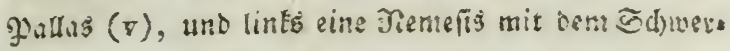

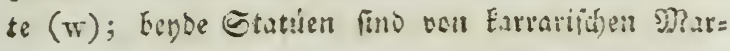
moi, von bem ebengenannten italieniifen Bitbǵaue: Erepelle.

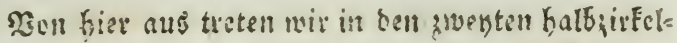
förminen Sogrengong; funo we: in iefien Mitre an=

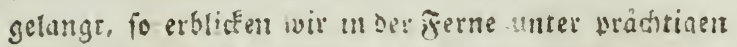
Wäumen den Eempel Mitwervens (S). Fr rulbt auf furintbifien Såulen, unb auf ber Nerserperte ther Dem Fingange, erideint in bulberbabener 21rbeit rie Bëttinn a:t einen গู?

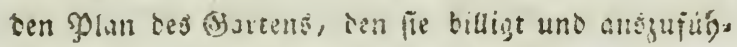

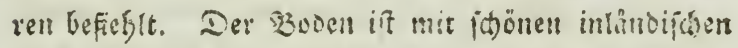

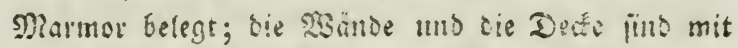

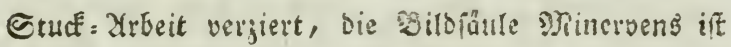

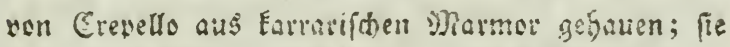
befand fich ebedem zu Dulfelderf, und if mit andoers Sunftwerker blerker gebracht worden.

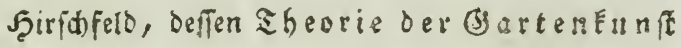

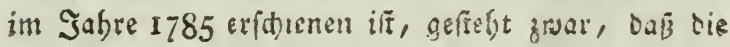

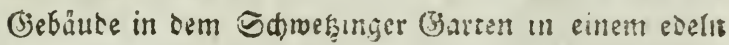
Gtyle auszeführt, uno alfo eine vorgh glid)e 3trote

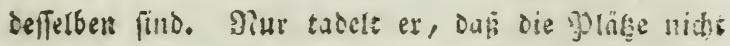

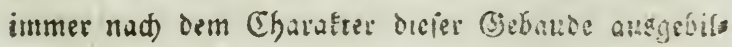

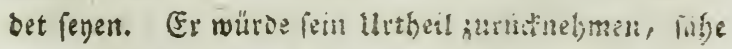

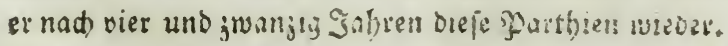

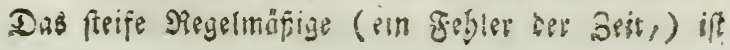




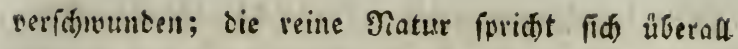
aus, uno wo mall dies nidt sbne greben seitenaufs wano benerfifieltinen fonnte, lieft man wengitens bas Etreben, die ängitlidsen jormen, die itharfen

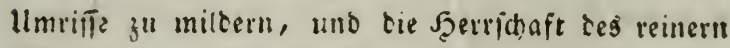
(jeid)marfes auch bier in fo meit "usjubebnell, als $\mathrm{eg}$ möglich war.

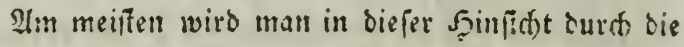
Umgebungen am Mhinerven = Sempel tuberraicht. Die abendtänciid)en glatanen fteigen bier mit lleprigkeit in bie Şäbe; erft feit fed) fie gevflangt, und idson baben mebreve cinen llmfang

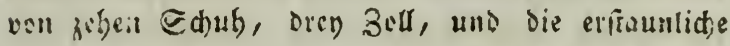

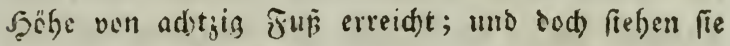
nut in fantigem Buten, unb man Eans folglid) Eeis

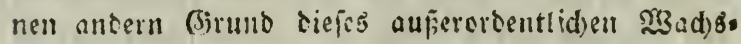
thums angeben, als bas fyurigentalwalier, weldes

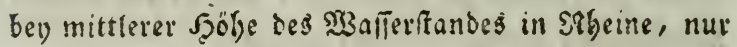

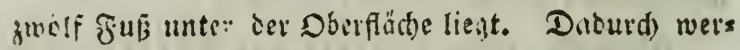
cen bie ticfen stsurzeln inmer frifd ergalten; unun= terbredien faugen fie Sebensfraft ein, bie fith sams

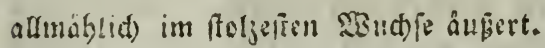

Dinje Erfdeinnmgen Geweifen übrigens unwiber=

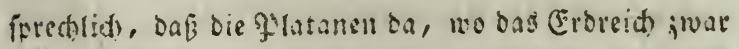

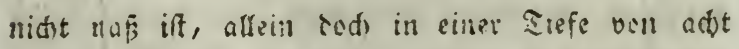

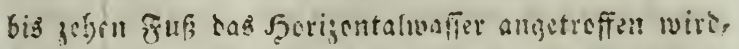
mit grofiem Shugen angeplanjt wercen Fönten. 


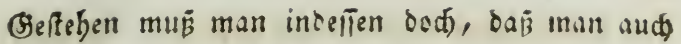
in ciejer Partbie ned manches fincet, was oer reis

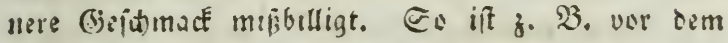

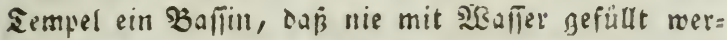
ben Eann, weil es Feinen Rlkfauf bat; es gebort alfo zu Den überpiufigen Dingen, melce unfie Bităttern fo geine Găuttill; fie batten nur Einn für Fradt, Die empadie Schonbeit firrach fie nitht an.

Sie qange Fflankung um den Sempel the net) nid)t den frepen, naturlicien (Charaster, mirurd) alfein eme folcte sulage idjen gerannt ju nerden rers dient. Ded nut wenig if ned a thun, io ni o cas Eteife uno Jengiliche oerfatumeen, und auth cieje burch ben id)welgerijhen $\mathfrak{S}_{3}$ ahs ifrer Umgetungen reizente Gitle wito mit dem Ganjen bumeniren.

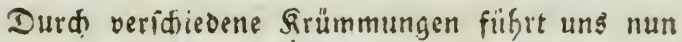
ein $23 e g$ von ЭRinerven Setligthume auf einen freyen, oan frảhtigen Båumen umąebenen Plak. XRer if die Eterbende, die ocrt unfre \$licfe suf fidh ztebr? Sreten wir näher: ha! es if 2lgripnina, bie Mitter Nero's ber im rabe die hlcern geäfinet werten. Diefe Ctatue it aus Farrariithem Marmer, gefwns

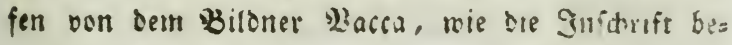
meifet: Andreas Vacca carrariensis Scu!pior. Sie ift das (jegenitüf zu bem shen beithrebenen auf

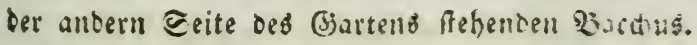

2fuf oiefer Stelle befincet fich aud ein Mirtur (o) und (m) eine Mineroa ganz abue Draperie, 
voll Furrariffem Miarmer ven Erevelfs. Beyde Bilby

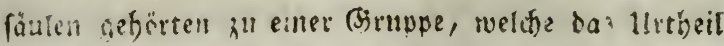
tes Faris vorfe!lte; Die brey anbern Dajugebiri= gen Etatüen ter Stme, sernuts uno ieg farit follen

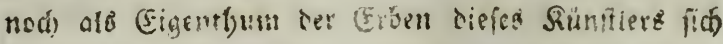

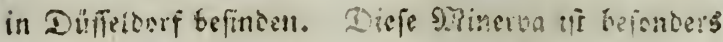

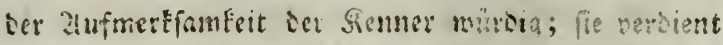

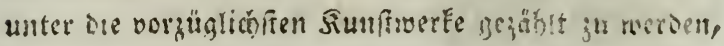

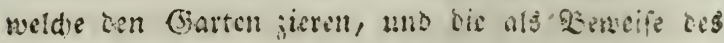
Enlentes jenes fitnfilers bis auf uns gefomen fint.

Nidt ferme von da fteben vier Baruffeilser van Eurrarifidem Mparmar (1), welde ier foren oft ges

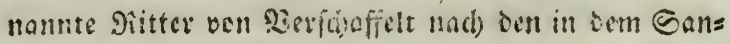
De ser Antifen gu glannbeim befindichen suffen ver=

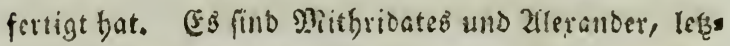
terer in bem "2lugenblife ber sefunumg, nadbem er feinen Jeind Sittuš ermerdet bat, bann Die Saifer Şabrian und Domitian.

Sabe bey ier Büfte 2leranbers ftefte ein afpollo aus weifem Miamer von iem wföihifhen Birbhauer

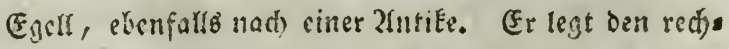
ton 2frm über ten Sonf unb beifit deswegen Apollo lycius, als Sarrifeber Der Encten. Diejer 2twollo und eine Ceres ven bemfelben Sgicifer, cbenfalts nads einer Zfntife gebiltet, waren ehemals in sen Pitter= inale ju Mambeim anfgeftellt, wo jesct sie Gtatüen Siart Ibesdors unt Estifabetbe 2luguftens feber.

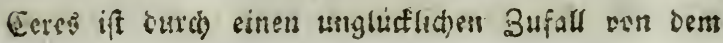




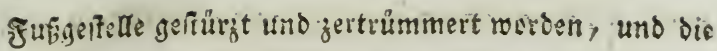
Eticfe murcen im $\mathfrak{B a b b a u f e ~ v e r a r b e i t e t . ~}$

Ége roir neitel gehen, müffen wir ben Manen

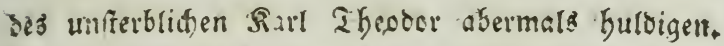
Itle fo eben beiduriebene sititen unt Etatuen fino

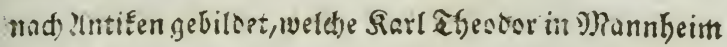
fin bem fogenantien ?Inttferiaale bat aufife!len lafien. Bereint fano man bier in voutrefticten Gingabgüffen Romb uno Griechenlands Meifferweike, "iebem Freuns De der Sunft, jetem Berveistert war der Fintritt uno

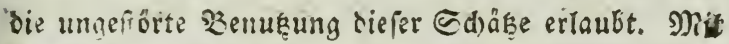

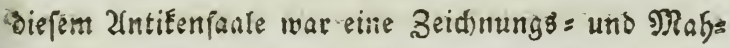
ler = llezdemie verbunden, oie unter oer sufflidt bee

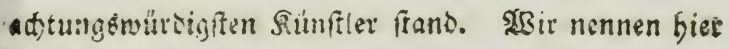

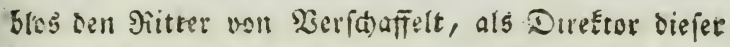
ZlEademir; ifm folgte der Direttor und Birthauer Ramine, gegenwartig in Mündhen; als grofefforen

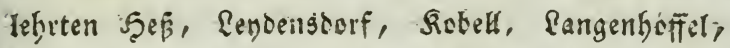

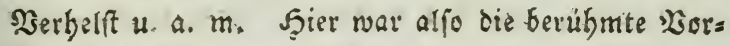
fdule, worin in einem Beitraume von zeben Jabren fii über jwengunbert iunge Sünitter gebildet uno zum Stubium ocr Junft auf Staliens Elafilifhem $\mathfrak{B} \mathfrak{Q}=$ Den verbereitet haben, und mefrere von ifnen wers ben in ber gebitdeten 23 elt mit bober Zlthtung genannt.

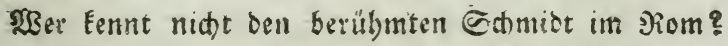

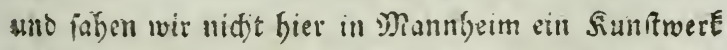
von bew genialen Dlacco, Der aud) ein Bögling ienes (edule ift? - 
Die politiffhe Serăndernng, die unjer Baterlano

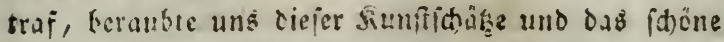
Inftutut erleid. altein nid)t lange beflagte Ilann= beim Diefen Xerluft: Siarl grtieorict), der Senner,

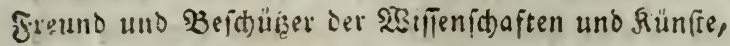
erfezte uns bald, was wir burd) die Ėreigniffe der

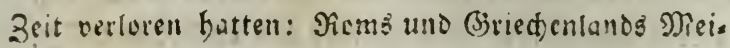
frerwerfe prangen in veríthenerten Pachgebliden wieder

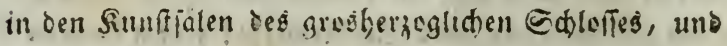
wercen ned immer ourd) neuen 3uwads vermebrt.

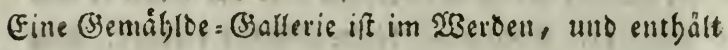

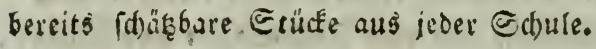

Einit nannte man mit Fechte Mannheim das Deutitue atthen; es fieft unter dern 3evter des weifen Siarl friebrich feiner Wiedergeburt entgegen. Die Stürme der Beit baben das (Bute nid)t tilgen fönnen, bus mall nadh überall fincet. Fürwaby, eg ift idbes rev bas Bute, wo es einmahl 28 urjel gefafist hat, zu vernidten, als oaş Schled)tz. Die Bewehner Mannbeims baken bey allen $\mathfrak{s}$ iderwärtigkeiten thren geläuterten (jiefd)mact und iffen reinen Cinn für das Edöne treu bewabrt, und boffen, das goldene Beit,

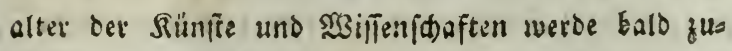
rủaf́lebren.

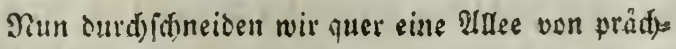
tigen Picb́aftanien, und treten in einen $23 a u m g a n g$ von Distbtannen und Rerd)enbảumen, weld)er juв Nof́dee $(R)$ fübrt. 


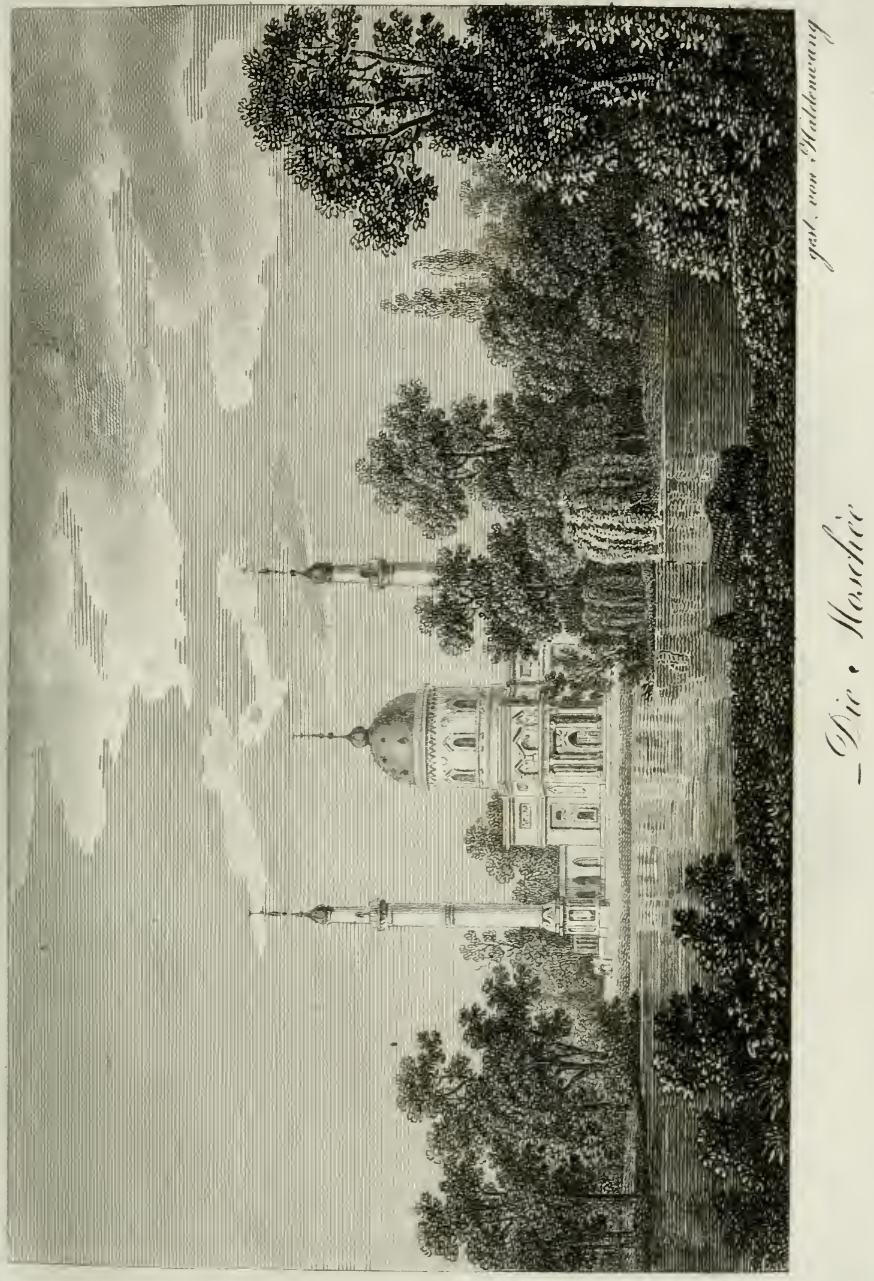



Şier nimmt uns cin rierecfigter, oon einem türs

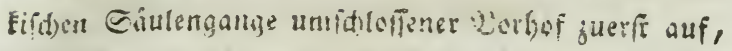
uno faton am Eanģange lief't man Dentfprüăe, bis nad) arabijder Eitte tiferall, angebradat fint:

\section{In ten Eoinmertagen \\ (E) Der limeije gleid).}

M্灬ুegen der sroje

begieft man die Demen.

2uf ber Mauer innendig in Sorfafe liept man:

Einfamkeit it befier,

2tlo boje (3efellid)aft.

Reden if Eilber,

Edoweigen if Giolb.

Edion die 2fnfidt diefes türEifhen Tempels aus bem 23orbofe ift impenirenb, und war wobl werth, von dem idhon oft genannten Şalcenwang aufigefǟt und darigeffelt zu werden.

Die Mroidee felbft ift ein Fradtgebande, defien frolger Dom majeftütific) fich erbebt, uno an Deflen esingange man die Gittenfarubthe finder:

Ein Eafter des Sisijon

(jilt fúr Taujent. 


\section{Wedfiet in ser Freundifaft Şringt Serberben.}

Das Innere des (sicbäutes entikribt dem nufiern Blange; ber Boben ift mit inländraten M?armer bes

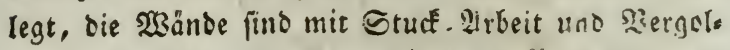
Dungen verziert, und überall find Ienfiprüte ange: brad)t. Im untern Sgeile ber Nofdee fteben fols senbe:

Der Ifor hat bas J̧erz im Munie,

Der Weife bie Zung' im Seergen.

20itienifaft ift eine Sirone,

Berftand cine golbue Şalşoierbe.

Reidfthum und bie 2 Belt vergeffen, (3)ute Şandlingen bleibelt emig.

Erwirb oir Gold, fo viel on Graudift, Uno Şseisbeit, fo vuel ou Eanmft.

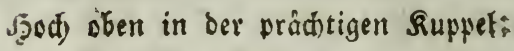

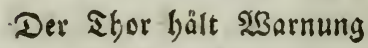
für Feindjhaft.

Wer alles begebrt, gebt leer aนช. 


\section{$-53-$}

Der gicio rubt niemabls.

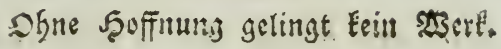

Jiefe bie Fautheit,

file bringt Sthaden.

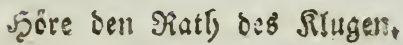

Seriatuiegenteit

erruitbr Riebe.

Riebe ben Fleis,

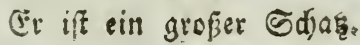

2tuf beysen Geiten des Domes if ein Sabinet, verjiert in morgenländifdem (jeidmate, und unter sem Gäulengange fino jwen Sakinethen von Bgypss marmor uno Stuck = 2rrbeit.

Die beyden Minarete vollenden bas Gaanze; eine

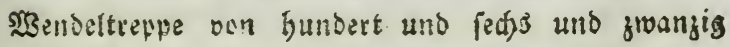
Etufan fülft auf beren obere, mit einem Bitter von

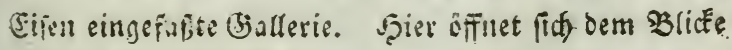

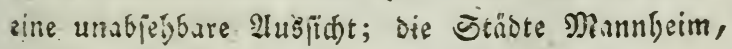
Şitbelberg, Spener, Sisoums, unjäblige Dörfer, 


\section{- $54-$}

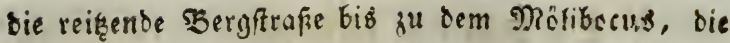
fernen Sogefen, bis fum Donnersberge liegen wie auf einer Siarte ror oem Ed)auenden ausgubreitet.

Diefer tưfifithe (3arten ift zun Sheife rrit ggaus ern umgeben, sie aber in innern Sezirte Durd Fflangungen bedecit find. In bieier Spartbie ift aud ene fleine Erderbobung, an deren \&ufe ter Weg nach Farlorube vortey gebt; orn bier aus ibberfiebt man mefrere Dërfe:, Jetrer und sisalsunaen. Eei.

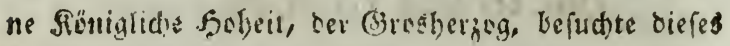
freundtiche weben vie Ruftchen lieblid)er, eine erfrifdende Siuble empfärngt ver Maller, Imo nur ber tauiendifimmige (J) fang ber Dögel unterbridt bie feyerlide Stifle ber. Llmgebungen.

Diefer berrlide Semwel Maksmets if fo tren im

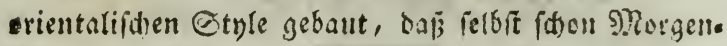

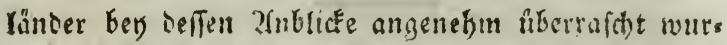
sen, uno fie fich beugten vor bem stamen bes allers bödifen, oer alb äufiere Bergierung in araficisen Sdriftüugent ifnen vergoldet entaenen frralte. Sreu Gat Saldenwang aud Dieje 2 infid)t mit alen ibren Peiţen und Eigentfrimlish Éiten bargeftelt.

Sritt man enolith ourd) bie grofe Sorbertblite it

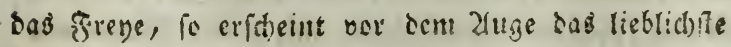
Eandid)aftşgemäbide, Das benť́ur ift. In siner mis

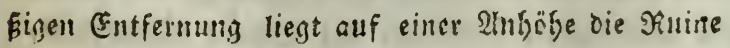

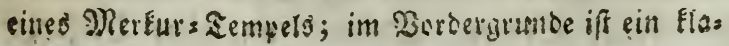




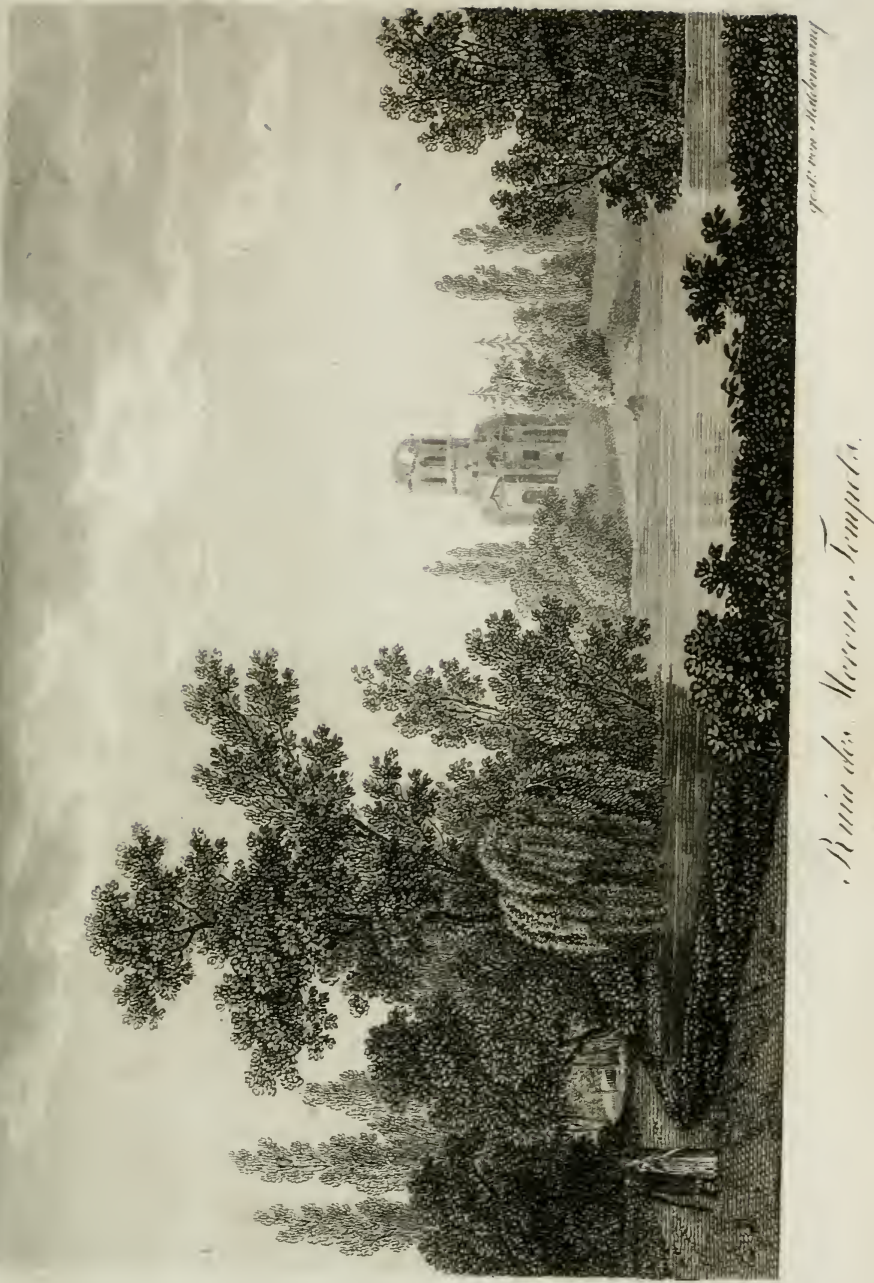





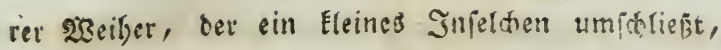

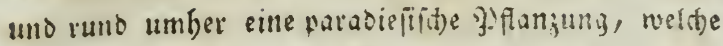
bie tiefen Einfighten in Die (jartentumit und dent reinen

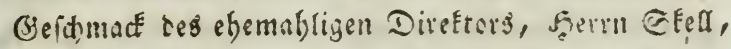
beurkunden. 2atle bieje Begentinante invegetn fid) in Dem Eryftallenen seidie, uno geben dzill Ganzen

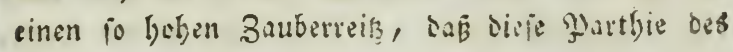

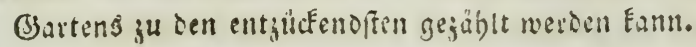

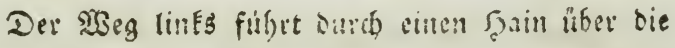

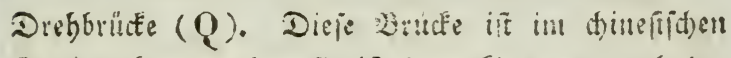

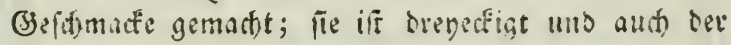
Fanal theilt jid in trey atme. Sit man auf ber Jatte der Brubfe, fo Eamn fie getreft werben, fo

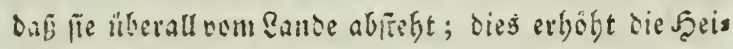

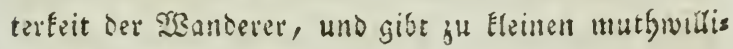
gen Diefiereyen Zlinfap̃.

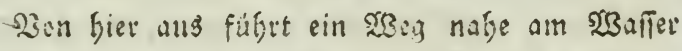
Gin; Der unbere aber windet fidd) burd) ein Eleines

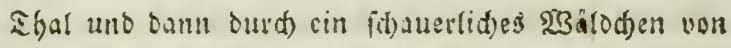

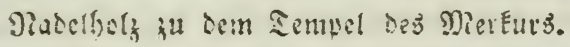

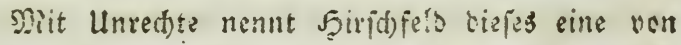
Shimmel berabgefallene egyntiơde partbie, und den Eempel ein Monument des Fönizes Sejoffris.

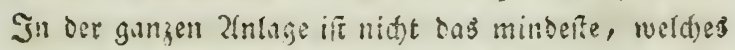

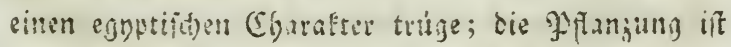
vorteflich uno binreisens lid)än, wenn man auj einem

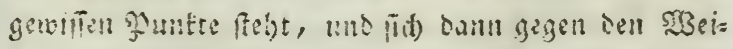

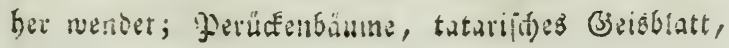




\section{$-5^{6}-$}

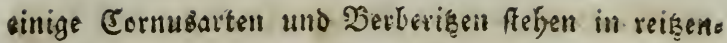
ber Mifidung; binter bieien fteinen in ounflerem.

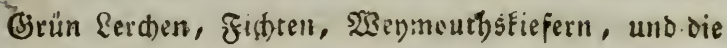
glübenten Wonelberen enipor, unt im Santergrunde. begränz̧t die Meichee, seren vollen 2lablict man bat $p_{0}$ bie Ecene. Dieje herrlidpe Jarthie ift das gange. Sabr hinourd fdoen, und man Eann fie mit Siectre für bie gelungenfte im ganjen (Sarten etÉtärets.

Eben fo uenig ift diefer Zenuel cin Mumunent des: Sefoftris; er if dem. Sjertur zeveift, dus bes weilen der Sarp des Bsttes, und die slintbe der rers folgten $3_{0}$ und ilyes f̧üters $2 \mathrm{~kg} u$ s, welche in balberbabener 2rrbets über ben Eingüngen vorgeterelt ift: zuch ben andern sorrwur Sciridffios wersients biefe. Faine nicht. Ex fagt, es fey nicht wentger, als eine voll ber Zeit allfgericbere Tiuine; hicr fats?

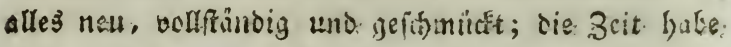

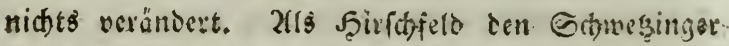
Barten befudxe, wavd eten an diejem Iempal gearz beitet, und alfo fontte die Siume den Ebarafter nod) nid)t Gaben, den fie baben muaje uno je(zet in io. boben Givede bat. Die Salffte der Suppel if einges

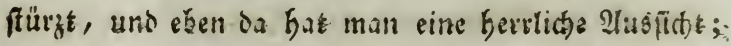
bas Chabute rufk auf cinem. Bervölse, in bem ein.

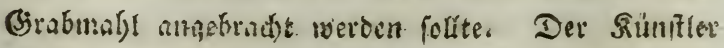
Şaldenwang bat diefen Biegenftuns in fainer geiftrek? Gen Manice burgeftellt. 
Futh in biefer 2fn!age rotd a:n 9ten ङeptembet Seg Inmejenteit des fiöniges und der. Fímginn von (exweien ein feit gegeben. 21!s bas feft in afpels fons faume geanoigt. rocr, eilte alfes zu der ganz

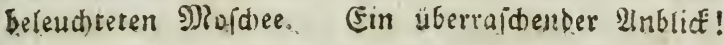
Die taujeno und abermafir tau[eno flämundje:t glänzten in oẹm iplegelbellen $\mathfrak{W}_{3}$ iber wieder, uns beleu(s)tete! Das Inannichfaltige Grün. der Umgebungen jaukerifh

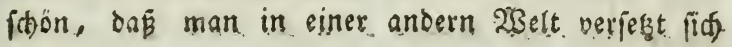
glaubte.

Der talentoulfe Sefmablex, Siarl Siunţ, bat diefe Ecene, von der nod) Jebermaun mit. Ent iutefen

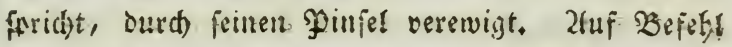

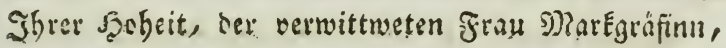
mablte er bie beleudtete Moid)e ouf. Seide., als Pod)tfüt zu cinem, Samin. Sdirme. Sein 2 ater,

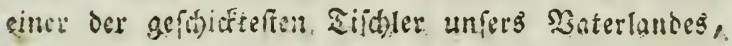

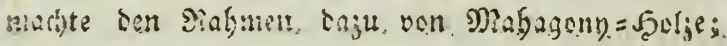

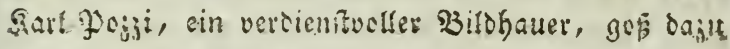
vier (A)lafende Genien in \$\$!ey, die brongirt - unten

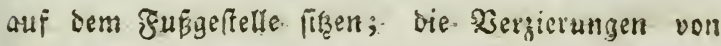

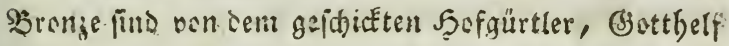

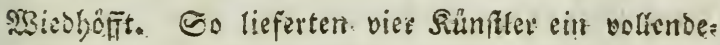

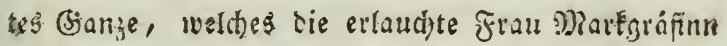

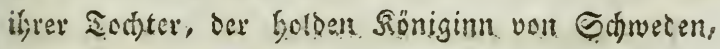
als GeIÓERE Gefrimmte.

Son bier aus Eann man mun zwen veríniedene

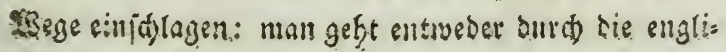


foten 2rmtagen, ober wäblt ben Eürzern $23 e g$, ber ju Dem gref́en Saufin füfrt.

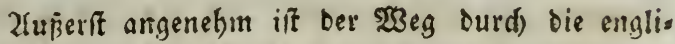
fhe Untage, gewöbntid) ber englifole (3arten gentunnt; man geft neben einem reiţenten $23 i e f e n=$ thate bin, zrwilchen abwedfelnten Saumbruppen. Siommt man bem grojern Baffin gegentuber, fo fieft man ther baffelbe weg curd oie berrliden zalleen auf bas Sdbloz, ein zlnblict ber prädy= tig ift, menn er burdh bie $23 a$ fifertitalen ber verfofies benen cerringbrumen verichönert wirb, bie nie leuchs tente fรünkd)en emporifeigen uno nieberfallen, un oon fpietenden Bepbyren in ber Euft verffeut werden.

S23endet man fith auf bie entgegengeferste Eeite,

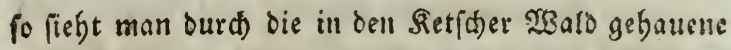

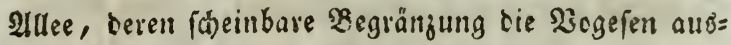

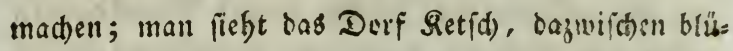
bende Fluren uno im (jan;en cine berrlid)e Eansidust, Deren Gefammteindruct überrajhend und ogrọ ift.

Dies ift bie lidjte Etelle znifhen ber ?nhage, bie von bem MRerturtempet hergiegt, und dem engl f(ben (5) arten, in ben wir man surd) sinen bu= ftern, von Goben $\mathfrak{B} a$ umen "uberwolbten sis

TSir geben an bem Schiffgaufe verbey, uns Erm. men enolid), nadboem wir eine beträd)tidide Etreffe

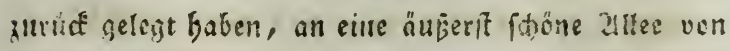

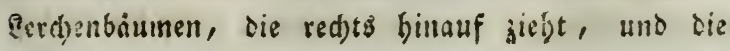




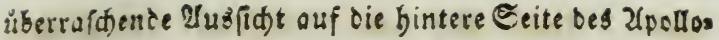
sempels̆ geruâbrt.

Die felteniten oušlåndifa)en Bäume und GSefträu. de fund bier mit tiefer, Sadfenntnif uno zugleid mit reinem (Sejamacfe angefflanat. Jebe lichte Etelle

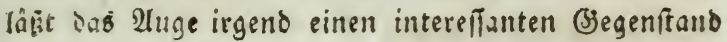

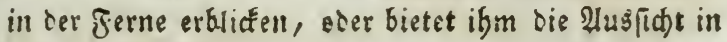
eine lieblide Randidaft bar.

Die cinzelnen (अruppen find mit Finfiat geortnet,

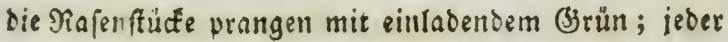
Baum, iede Ctaude erregt Benunberung durch ithwels

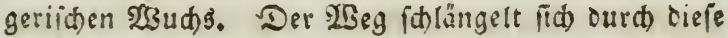
reithe Planjung, uns bey jeder Wentoung ergengen neue biegenfånce Erftaunen.

Enolid) gebt man bey ber Saumidule ủber die ss:üfe, tritt in bie föone allee von wilden Faftanien,

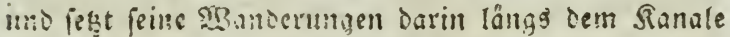

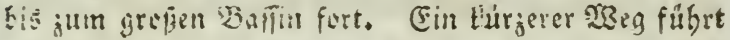

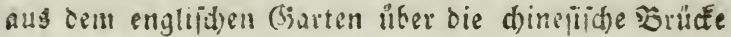
(h) auf Denjelten sount. Ein anterex $23 e g$ geft osm Ismpel bes Deserurs turch bie Snjel über jwey Diruffen ebenfalls an biefes sallm.

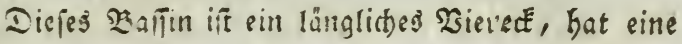
ganz regelmẩige frorm, uno if mit gefaranen Stei= nen cingefapt. Niur in ber Mitte befferisen auf ber

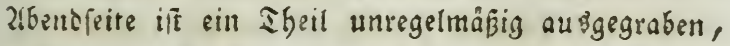
4no gefumactodl gecronete Gruppen volt Irauers 


\section{$-60-$}

weibell, Elfern und anbern Băumen, ziersh feins Uffer uno geben ifm ein romantifdes 2fufeffen.

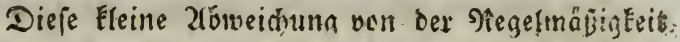

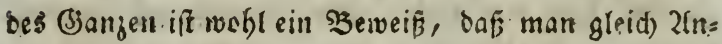

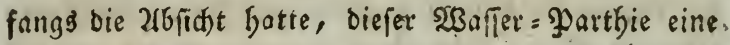
idjönere (Gefralt gu geben.

Die ltmidaffung if nicht forwer; nur mus fiet. mit Uleberlegung und (Einfidft gemad)t, und babey. Sillififidt auf Die umgebenden 2fulagen genommen wer: ben; mit diefen mu man biefen grofen Sant in: Serbindung, bringen; bann mirb biefe Paattbie sinen:

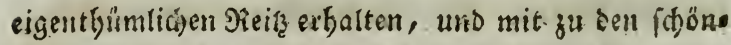

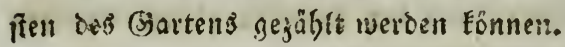

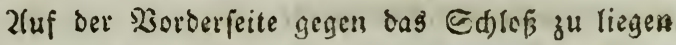

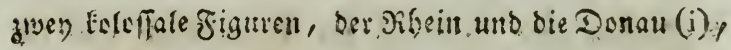
mit ibren Zlttributea, nus gelben übertbeinifder. Eandifeine vou bem Stitter von 3eridaffiflt gebauen. Die Giegenfeite biejes Ranales follte mit zwey äbnli=

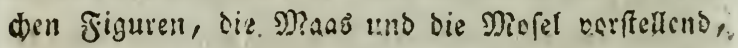
geziert werten; allein Strid) Den Iod Maximilian Ios

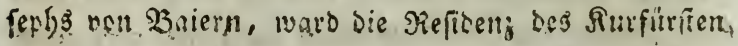
naci) München verlegt, wit auch von Beriduaffe!t,

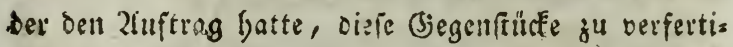
gen, waro bialdy ten Sod bet siunft entriffen, uns

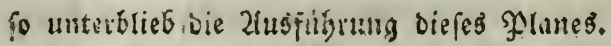

WBir geffen nun in die frenjöiliten 2 fnlugen A A jurtiç, und Eommen Fogleid an einen merÉnürbigen Dre. Jerate gu ber beit, wo man affes aufbut, siefe 
Znlagen ju verfdjönern, uno es aud bereits io weit

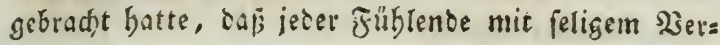
gnügen in dicfen weitläuffigen (Saäten verweilte, ward

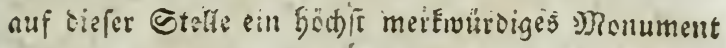
cutieckt, das aber mit ben reişenden Ulmgebungen

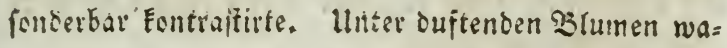
ven Bräher verborgen; der grüne Siafen beete Urnen, 2ude uno Befeine.

Serweilen mil ètwas bey biefen ernitern Begente "ftäneen; es roirs cem Rejer nifft unnulfEommen fent,

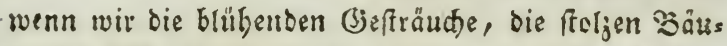
me, die Đ̧raditwerke der Sunt verlafien, uin dent Dentemáhtem des S

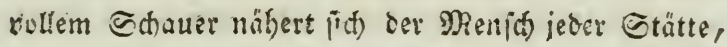

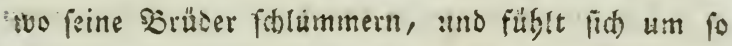
mehr erigriffen, wenn er an einer Stelle weilt, we $\mathrm{el}$ bie lleberreffe der Eapfem fintet, Die in grauer Sors zeit Eămpfend fä: Frreugcit und Saterfand gefallen find.

3u bem fint diefe Nadforfifungen nitit obne be= 'fontern Nuķen: Denn was fprid)t bie ভ̌tten alter SolEer und die Bebräuche ifrer Fetigion deutlither aus, als bieje (5rẩber? Darum eradbten wir es ber Mühe weith, etnes weitläufiger bey biefem begens ifanice zu pern.

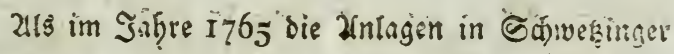
Barten weiter ausgedefynt werden follten, ftand ein

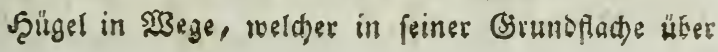




\section{$-62-$}

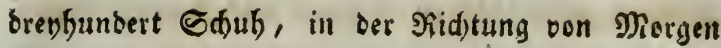
gegen ?bend, entbielt.

Damals welle man niфt bie geringfte Ungleid). beit dulden; alles muß̈te geebnet weroen; ess waro

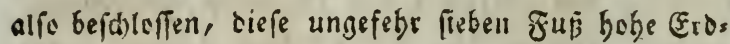
erböbung abzutragen.

Saum hattes bie 21tbeiter ungefebr vier Ethuh tief gegraben, fo lng eine lange Fieibe von sodenige rispen vor ibnen, und fie fanden eine sisentge llmen mit afche und Giebeinen.

Diefe Sörper lagen in einer gewiffien Orbmuna; fie waren alle mit dem (Gefidte gegen Zluigang gemens bet, und ber Sepf eines jeden rubte anf einem steis ne. Die Utruen bingegen lagen chue Drinung zwis

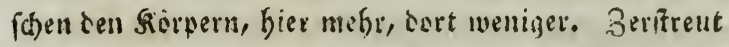
umber fand man Edwerter, Eanzen, Edritse, Zans gen, irroene und gläicrne Biefäse. Der Şüigel, fo weit er reid)te, bebect te Reidyen; und wo er aufgürte, verf(t)ano aud) jede Spur emes Begräbniffeß.

Dieś if das erffe Denfmabl ber 2frt, welches bier entbecft worden ift; das zwente fand man bas Jahs: Darauf einige Mieilen von ba. Beyde find ben Bjes

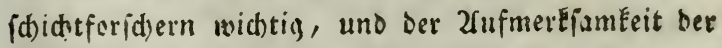
Oelefrten um fo mebr würbig, da einiges stdtt bas burd) uैber bie Bseidhichte bes zllterthung verbreitet werben Eann, die in Symilid)t nuf unire (jegend nod) meiffens in bas Dunkel der Ungewiskgeit sebünt iff, 


\section{$-63$}

oa bie Denkmähler ber Sorgeit beg uns etruas fels ten finto.

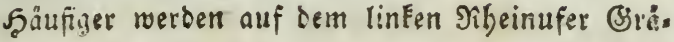
ber, Ulinen und Carkephage gefunden. Didh auf ter rediten Bilgeinfeite, und in Der Etrecfe, welche Der Pecter burdtäuft, finto Dergleid)en Entbeckungen fehr ungewöbnlid); berm in unferer (biegeno fano man

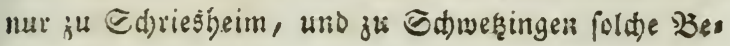
gräbniาị.

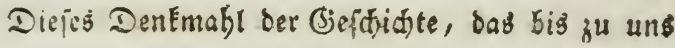
geEmmen irर, bietet fowoll in Şinfihtt auf oie Stelle, wo man es entoefft, als auf Die Ulenen, fiörper uno Intfrumente, die man befunten bat, reidhaltigen Etcif jum Padbienten dar. - Satte die Siatur bies

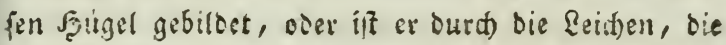
man bier eingeidartt bat, entfanten? Bieboren alle diefe Uinen, alle dieje Förper ju einem und bentels Eell Grabe? Doer mútica roir annehmen, Dás es fo viele veriticedene (stäber find, als Sörper uno Ur= nen? SWas if ton ben Edistrertern, Sdildern, fan, jen uno ben übrigen bier gefundenen Snitrumenten fu balten? Ilnterfudtt man biefe Dinge einjeln, to entîeben miberiprechente Sermuthungen: biefe bes

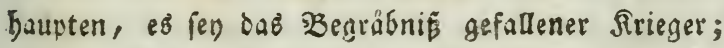

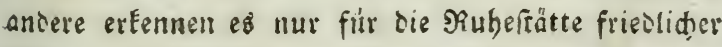
Berwolgner. Sene finden bier bie Gyuren blutiget

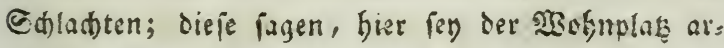
Eeitjaner zlufiedter gerefen; viele zäblen eg ju sen 


\section{$-64-$}

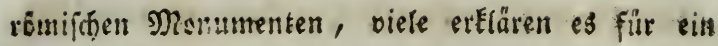
Deutides DenEmafl.

Die meiften vertieren fia fo ferfor in 23 ermutbuns gen, taf fie enolich felbft nidst mefor wifien, weldie Mieinung fie beftraupten fillen. Man verfuctste, arre

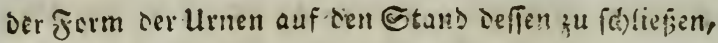

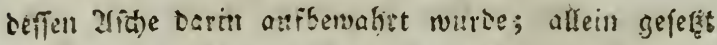
auth, an ben Urnen fen eine gersifie zusigeidnung

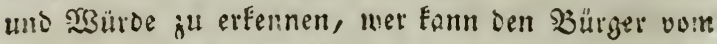

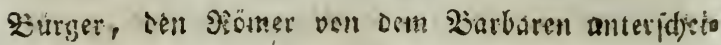
Den? -

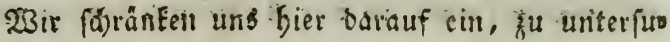
den, ob tiefes Dentmalt ein Friegerifdes ocer ein

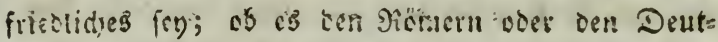
fdien zuseftrieben werben milfe.

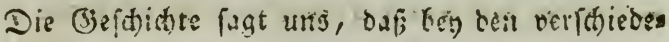
nen Bälfern de: Bargteit die Biebräudte bey Begräe, niffen eben fo veridhesen waren, als bie Gitten. Serfdiwenterifder 2ufwumb berrfdte bey Beeroigun:

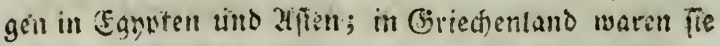

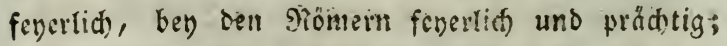

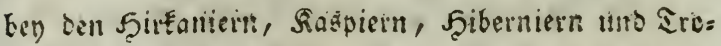
globsten war bey folden Antäfien Lumenidslid) Eeit

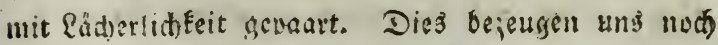
(Eapstens Jyyamiten, Zfiens Maufold̆en, tho die DenEmátiler Reins unto Briecthenlandos, felbft die Ret= denbegängnile ber Sentben uno szarbaren befätio gett $k$ th 


\section{$-65$}

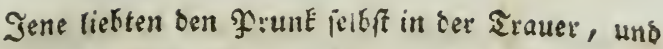
arridteten nidft fowohl ben Scoten, als fió) jelbit bie ftoljen Dentmäbler, die wir nod anftaunen Sie

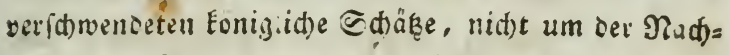

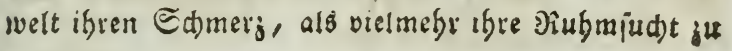
verfünden.

Diepe Eannten Eeine prunEvolle Maujeläen; die Grabbügel ifrer Beriferbenen trieften vem Blute bee

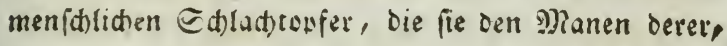
die ifuen theuer waren, würgten; 23 unden und ferbft Der Eoo waren bey thnen Bewelie - 2lusoruce dee Erauer.

Die Ecythen veraditeten ben damals yon allent gefitteten SölEern angenommenen (Biebrauch, Die Bies

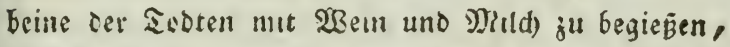
fie zu falben. Bewitr;e, Srenen uno Fränje ibnen it

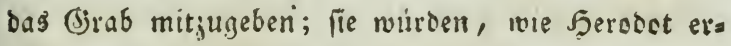

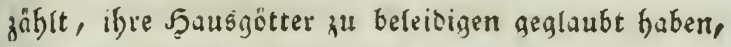
wenn fie nidit ein Etuif oon dem Dhre gefdnitten, Die Saare altgatallft, sie Zfrme vertentt, Stime

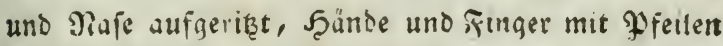

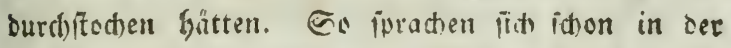

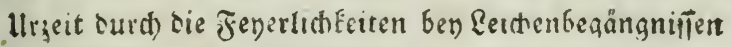
bie Eitten uno rie Neigungen der $\mathfrak{B o l t e r}$ aนs, uno fo iprecten fie fid) nech aus. Siste aber bie Eitten, Bebrüuche und Geereşe von einem Bolfe bem andern find überliefert werden, fo baken lich aud die (je= bräucte bey Seeroigungen fortgepflanģt. Son bent 
Gaptiern empfingen bie Griedten, von biefen die

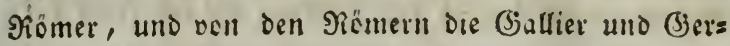
manen ibre meiften religufen Sitte. Ded bat jebes

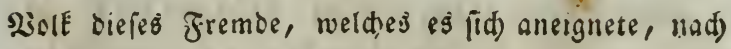
feinem Nationalgeifte gemocelt.

Seine Denkmähler fagen uns, welche Gebrăucte bey) Beerdigungen in Bjallen und Biermanien ges berridgt baben, bern es find Feine uns aufbewabre worben; inceffen geben uns gleithjeitige Sd)riftreller

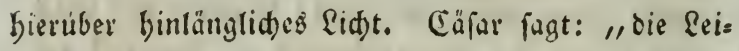
d)enbegängnifite Der Gadller jind glänzend und Eeffbar."

2Bab den Berfierbenen um Reben theuer gewefen, wutbe alleż auf ben $\subseteq$ d)eiterbaufen gelegt; felbit dev Sined)te uno Dienftleute, dia fie geliebt batten, fitono te man nicht; fie wurben mit ber @eide verbrannt. *)

Die Deutfden hingegen, wie Sacitug bejeugt "\%), Iegten gar Eeinen $\mathfrak{W B e r t h}_{\text {auf }}$ Fenerlichfeiten bey ifren Reidenbegängnifīen, fie betrad)teten ein grebes, mit

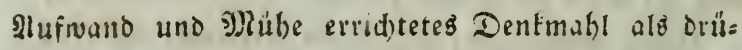
dend für die Toiten. Das einige waro becbact)tet,

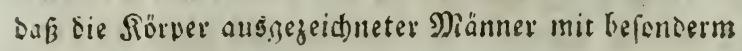
Salge verbrannt murben.

Den Eateiterfaufen bedecften fie nidte, gleidh ans Dern WötEern, mit Siteidern uno woblriechenten Din.

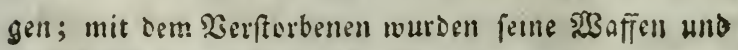

*) J. Caes. de bello Gall. Lib. VI, 19.

*i) Tacit. de morib. Germ. cap. $2 \%$ 


\section{$-67-$}

pein Fferb verbranut, und cin Graşbủgel bexte die Widie,

$23 a s$ Sacitus weiter von den Deutfich fagt, ges reidt thnen ju greper Fbre: "Shre Slagen veritum men balo, ibre Sbránen rertrufuen, doch pat erfit beren iff Sdmerj uno ifre Erauer auf. - Denn bey ilnen gejiemen Thrânen oen গ্̉eis bern, ben 准unern Erinnerung! - "

Nid)t obne allen (jirund Eann man begausten,

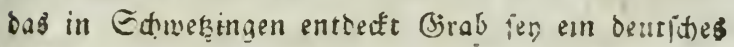
Girab; Denn allevi verfuret sie Eitreneminlt unjerer

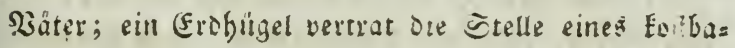
ren Monumenteg. Lllein wenn mu dre Gache uáber unterfuchen, wenn nu sie Gieitalt ber Edwerter und Sdirte, rie lleberbleibfel einiger Inithiften,

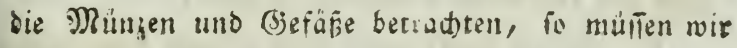
gefteben, daf́b dies alles on den Biömern uno nicht ven den Deutiden berfommt; dies berweien unwis

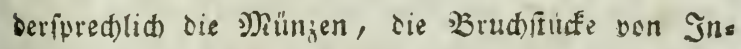
fidriften und mebreve romija) Snitrumente, Die man bier gefunden hat.

马war fino die Münjen vem Noft ber Beit betecft,

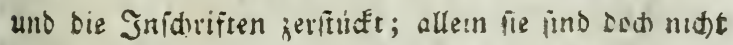

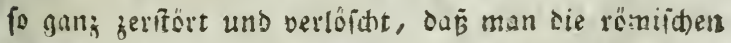
3udhifuben nicht erkennen foute. Mishrere Urmen find fefr gut erbalten, uno unter Diéen iĩ eine auss gejeitulet Durd) beflern Etrif und fdisnere form, melde auper Gebeuren und या由), die Eptse siner 


\section{$-68$}

Eanje, ein zerbrodjenes Sdjilo, eine jirEelförmig jus fammengebrgene Silinge, mebrere Bjtäfer, Meffer, Gabeln, uno einige Münien enthielt.

Eв war rämifabe Gitte ien Sobten verfdictene (jerâtbíchuften mitzugeben, uno fold)e zugleid) mit ifrer affhe in sie Ulmen zu verfdthefen. Darum findet man gerseghntid) in folden (Brïbern Eoftbare J2als = uno ?trmbänder, Simge unt antere Fileinsa bien. Der Rurus bey Leichenbegängnifien war bad, geffiegen; jeter wollte fie entweder nach) feinem Etana De, ster nad) feinem Sieiditgume eingeridtet haben.

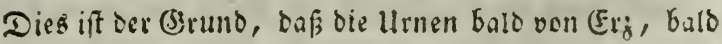
yen Etein, balo ven Ihon waren. Die Ueberrefte Srajans und ces Ceptumus wurben in goldene llmen, Die Bebrine tes 2llexander Eeverus in einen 2ifdens Fiug von Foltbarem Eteine verfiflefien.

In Stalien hatte man burd)gehenos biefe llenen non Erj, Blas sder Miarmer, m Den Folonien jens feitş ber 2upen ven Bbon oder Etem; auğer Stalien

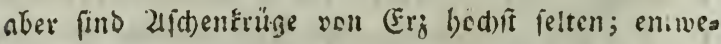
Der well mentg vernebme Siomer in den holenien Shre Eaufbafn bienieden geentigt haben, soer weit

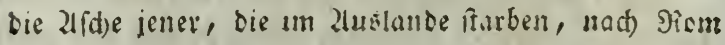
geiductit wurte. So frarben Irujus (bermanicus und Eeptmm Geverua in Strtumen, uno thre 2l(a)e waro in golichen (Betaben nad, sicm gebracht.

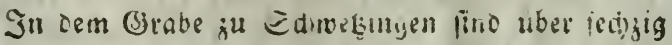
Htren gefunten roorber; alie waren ren Ibon, 
aufer ter oben beidriebenen, welde wen EF'; war, uno wabrifheinlich die llide bes इeerfübrers oder bes Sorftebers der Folonie enthielt.

3u Svever bat man 'ganj äbnliđe (jefäpa ents

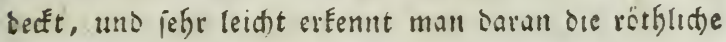
Erde, die um Epeyer mo Gdwethingen gefunter nirt. Jiebit cen Ulmen lagen mebrere jierippe, wie bereits gefagt worden iĩ, in einer gevilien Dromung,

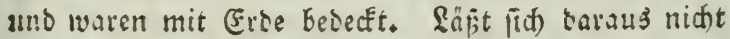

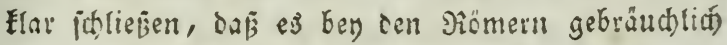
war, cie Eusten fowobl ju virkrennen, als jube. graben? Incellen glaube man nicht, baßj biejer ceps pelte (sebrauch Glos ourch Saune, soer veriấrte Cje: wobngeit jey singefügrt werten. \$Die Uriache biejer 2lbweidung if in ter sorzeit aufjujuchen, uns aus

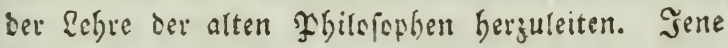

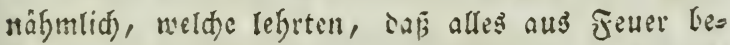
ftege, bebaupteten mit iem Jeraflit, bie Förper snübten aud) wieber burd Jeuer aufgelsist werten. Selle abet, bie das Eyftem des Shales angenommen

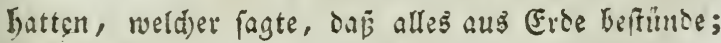

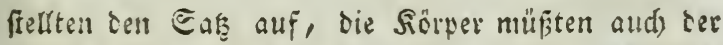

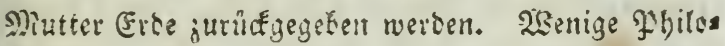
when rerwarfen bie Beerdigung, allein fefre viele ben

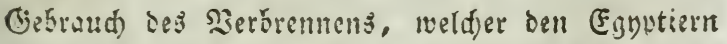

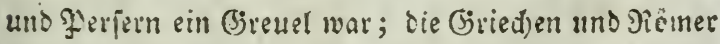

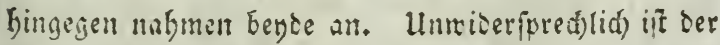
Gebrauch ber seerdigung ölter, natürlidjer, uns 


\section{$-70-$}

bert Sitten ber erften Menichen angemefifener. "Mir mentgitens, fant Cicero, ichsint die n̈lteffe Sitte ber 2eeriguna die geneien zu fenn, seren fith Cyrns bey Dem Ẋencphor berient. I Iaf Cyruş begraben wors ben rey, erzaglt Xencubon). Denn ber (Erte wir sie Setche wieter aeneben, und gletdjam mit dem Biewande der Mutter bedectit."

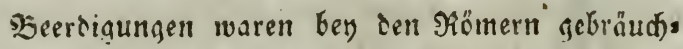
lidser; uno zu der Beit, wo die Siewnblik nidt surd innere r riege erfoúttert warb, Eanute man Eaum ben (jebraud) oeg Berbremeng. 2lls aber innere Zwwiftigteiten bie Bjemutther erbizzten; die raenten Büraer rie feithen aus ben Giräbern riffen unb bes fdimffen; bielten es die giömer für fiderer, die sooten zu verbrennen. Grilla, aus bem (jjefibledete Der Fornetier, Der aus mütbendem f̧alfe cen Förper Des Marius aus feinem (3rabe berverboblen und unter

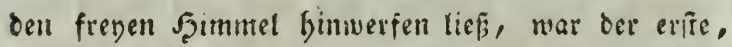
welcher nach feinem Sove verbiannt ju werben vers laingte; dom er bejergte, die fretunde des Mrarius mödten (jileides mit (sierd)ent vergelten.

Snceffen mar der (sebraudb, dic Ecoten ju rers Erennen, bey oen Sämern nidte neu. Numa f'om, piliug verbut fach ourd) ein befonteres (jefera, die

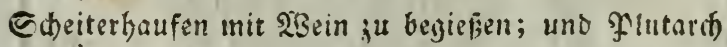
erzähit ooll ifm, er babe Eurg or feinem Sode vers eronet, nan folle feinen Sïrper nidt berbremnen. Daf der zrwizache (Jestaud) Der Beerdigung und bes 


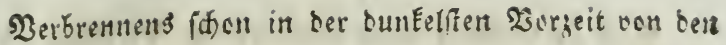

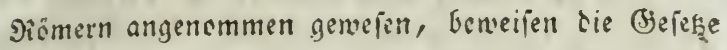

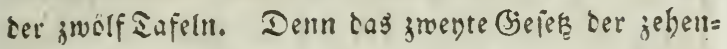
ten Iufel, von bem beitigen Piedte, fagt austrủete Iid): "Ein Eciter foll in Der Etnot weder begraben, noch rerbrunnt werden." - Es war alio erlaubt,

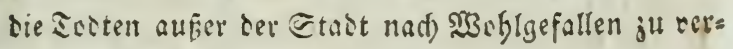
bremten coer ju begrabert.

Dar bieje Sitte fin auth bis in die Munijinat= ftabte und Folenien verbreitet babe, bemeilen die Gräber, bie mast in JranEreidt, Epanien und Deutidy= land entereft bat, werin buld Sorper, bald afiche, balo llmen, balo @arkephage fino gefunten wortert. 2(ber bä́) felten trift man in einem uns bemjelken Brabe, wie in dem ju Ethweß̧ingen entoeften, Siör: per unb Srauerutnetr jugleid) ant.

3war pagt Sielin in einen Edreiben an \$onts faucen, daj man in Cimbrien, DännematE un

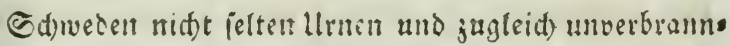
te Röper fince. 2utein bier ift bie Nieke oon Cim Erifiden $\mathfrak{Z}$ egräbnifien, uno nidht von rëmifiden; unt weder Sfetin, ned) Mientfaucen füfren ein Ienkmabt ber Römer an, sas bevoes entbalten bütte. Bjewifa.

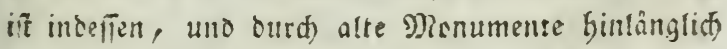

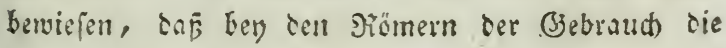
Icten zu Eeerbigen unb ju verbrennen ju berfe!tent Beit unb an bemperben Drte zugleid Sep̧anten bake. 
Das in Garmesingen entcedte Brab, wenn wir eb für ein römiface erElären ourfen, bietet uns sies

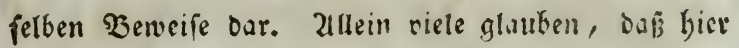
Ranichen von zwey verfderenen 3 olkern ruben; fie behaupten, bie Urmen entbalten bie zlfije rou Sicmern, Die antern Eeschen feyen Barbarent.

एक

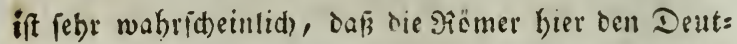
Fden eme Sod)ladt geltefert haben, uno baj man bie Befallenen auf Dem $3 a b l$ plakge begraben hat. Diefe $23 e r m u t b u n g$ geminnt an 28 abridreinlid)feit, wern man die Rage deș Dites betraciter; Denn nidst weit ben bier war Aita ripa (2'Itrip) ein feffer ven ben Sismern beiek̨ter fiunft, voul dem fie ausigingen, Die Sewobner des jenfertigen Sibsunfers uno am Nerfer anzufallen; Dies ivar gleichiam bre Fifurte, burd) welche fie in Deutichlant eindrangen uno bie

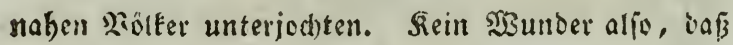
bie Deutfchen alles aufbaten, ben allgemeinen zeino won ibren Grönzzen żu entfernen, uno daj bartnácfige uno băufige Biefedte zwifthen ifnen und den Siömern vorgefallen find.

Daber ertarten die meiften ein foldes Grab, fo bald fie sarin Sd)werter, Eanzen unt Sd bitse fanden, für bie Siubeftätte gefallener Jirigger; fanten fie \&ei= d)en und Urnen zugleidy, fo entidsieden lie slme bes: benken, jerre wären bie Heberrefie ser Ueberwante= nen, und biefe enthielten sie zlfiche ber Eieger. Die römer nämlid, fagen fie, bätten ifye నubten vers 
Brannt unb beren zfine in Uinen aufberwabre; nie aber bitten fie oen beliegten Baitaren gleiche Egre guerfistnt, fondern fie mit Erbe besectit, um fie

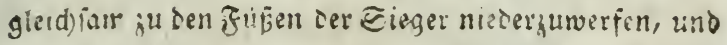
aud) nad) ifrem Sooe ned) thber jie ; triumplinen.

Diere Meinung war and nad) ber Entbecfung ses Grabes, in ๔đwekngen die übernoiegende. Man

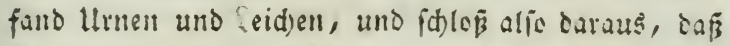
biet rie Cieger und die Eefiegten bey einander rubaten.

Das Ientmahl, weldes Siarl Iberes auf bie: fer Ctelle etridten liep, fpridst doeje Meinung bes fimmt aนs.

Xuf ser Sorderfeite ไiej't man:

$$
\text { Martis et Mortis }
$$

Romanor. ac Teutonum

\section{Area}

\section{Inventis armis}

Urnis et Ossibus

Instrumentisque a!iis

An. MDCCLXV. detecta *).

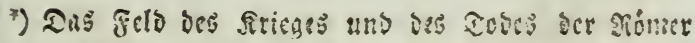

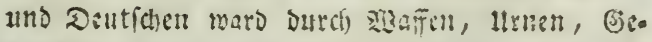
beine und altors Snitrumsite im Sabre 1765 cnto DecFt. 


\section{$-14=$}

Juf ber anbern Geite ftebt:

Pacis Artibus

Vitae Suac deliciis

Aequato VII. pedum Solo

Vindicarit

Car. Theodorus EI.

et II. H. P. C.

MDCCLXVIII. *)

Son biejer Mieinung milfen wir abweichent; nidit als ob wir bie unfrige fin bie teffere auforingen woll= ten, fondern weil ung Giủnbe beffimmen. Ilns ers

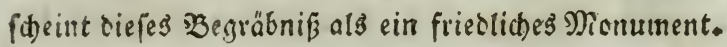

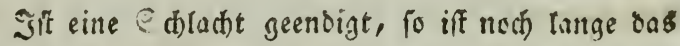

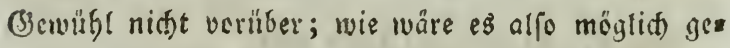
wejen, die Siorrar to ju oronen, wie fie gefunden worben fint ? J̦̈̈tte mant 3eit gebabt, bie Gebeine uno zlfiche der eingelnen in Umen jujammeln? ober aud) nur bie lleberreffe ber Sorgefegten ourd) Xridens Enüge von ben übrigen zu unteriäeiben? Mentfaucon

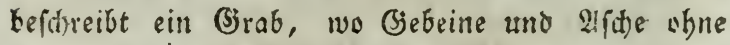
Drìnung aufgebâuft noren; dies ift ein Bòneis, daß getstiebene Siteger nach einer Sdylad)t bort find eins gelidarte warden.

*) Den Siunften bes firtebens, ber Şonne feines Set bent, hat Sart Sheodor diefe fieben SHE boch ab. getraaene Etclic mieber getbeift, und biefes Denf. mathl gejertit izeg. 
So fant man bas Begräbnif in Codregringen nidt. Gebeine uno 2liche waren forgfältig in Urnen gefammelt, bie Särwer lagen nidt zufallig unter eins anter, fontern in einer beffimmten Meibe, wie es bey bürgerfiden und friedfidden Beerdigungen becto adtet mart.

Eolon bewiek aus ber Suge ber Sörper in alten aufiegratenen Micnumenten, Daß Salamin unter die Serriduft 2tthens und nidht Megara'a gégërt Gabe.

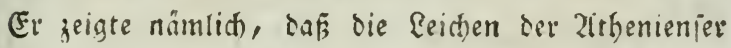
alle in siner gevilfen Dronung lägen, Die Meijarent= fer aker unter cinanter genorfen begraben wären,

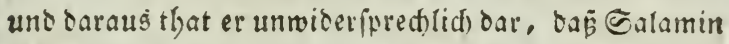
mit allem Piechie ven den 2ithenienjern in wejig ges nemunen worden fer.

$\mathfrak{M}_{3} \mathbb{H}^{2}$ wollen uns Eeines andern Beweifes gegen jente besienen, welthe das Grabmaht in Sthneringen

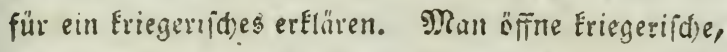

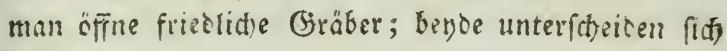

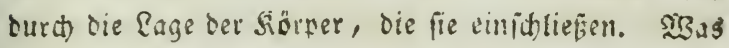

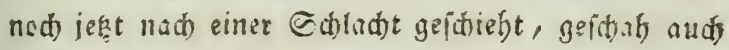
Damahls: sie Gjeblitbenen wuten shne Unterichied in sire Brube gawarfen uno veriftart. Saum dar

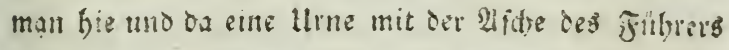
finter, sa fingegen in ten antern Grabunighern fals de băula angetrefien werten.

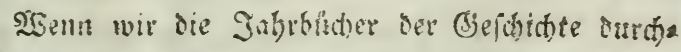

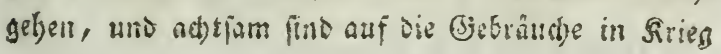


und frieden, fo werben wir uns überjeugen, baf es nidft anoers sernn fonnte.

(5) war grwar ben ben Griechen und Siömern ein uraites Syerficmmen, nach) ber Edjactit ten Bjefalles

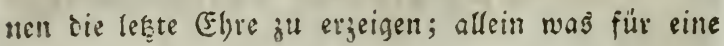
Egre wat bies? Setne antere, wie Sirius, Flerts, Eacitus und şlutaid) bejecugen, als baf bie Evoten inseiammt verbrant wurben, coer of af fie alle zufammen ein Girab aufnugm; wic jente Expartaner,

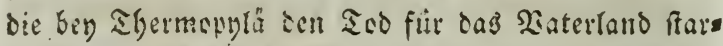
beir, nur ven eille m Şügel bedect't nurben, uno nue Surdi eine Infidrift ber Sort bejeidnet wurbe, wo fie rulgeten. So wurden bey den Siomern die bुes beine dreyer Eegionell, gleich Serwanda tell, in eill Grab verifhart, oa Eeiller wuste, ob bier bie Erde Ueberrefte frem der oder der Eeinigen bedecte. ") Sutr die Inführer wurben z̆ Zeiten von ben gemeinen Fries gerit unteriftieben. Eo erridhtete Eumenibes einen

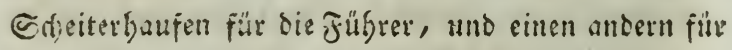

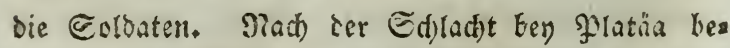
Ftimnten bie Qacebömenier ein Girab fü bie Sriefter,

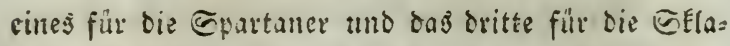

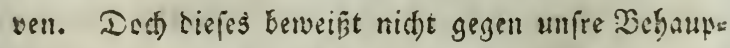

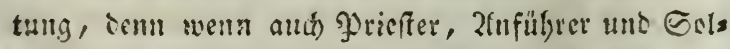
baten auf bejendern Echeiterhaufen ve:bramt wurs Den, fo war es bech nioft möglid, Die Gebeine uns

*) Tacit. annal. Luib. r. cap. 62 . 


\section{$-77-$}

2rje eines jeben einzintuen ju fammeln, wie man es

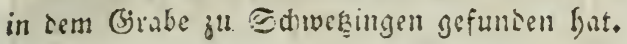

Şätte man nut hier unb oa cine llene, oder nue iene einjige oon Erg, die'wir sben bejthrieben baben entrefte, uno wären die úbrigen Sierper shne Drbs nung aufgethürmt gewefen, fo mulibten wir gefrehen, Dafi man alsiann irgeno einen Duerften soer Seerfúfla rev bon bem gemeinen Earbaten leid)t Gätte unters fiteisen Eimmen. Da aber alde Sierper eingeln in eis ner beftimmten Drinung lagen: fo it sies Girabmaff

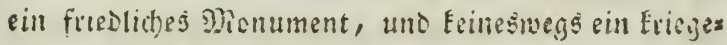
rijdes. Die in otepem (jrabe gefuntenen Ed)werter, Sangen und Edilde find fein Beweis gegen unfre Mermung. Dieje Eriegerifden Inirumente fino nid)t

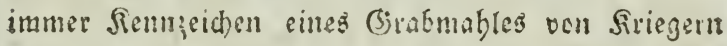

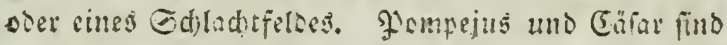
nidht in ser Ed!acht gefrerben, uno ocd) wifen wir,

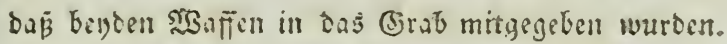

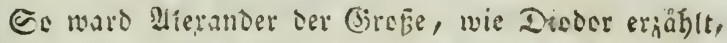

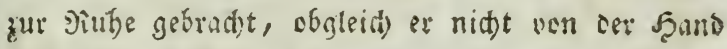
bes feinces gejallen mar.

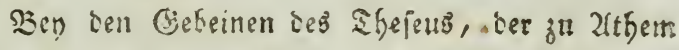

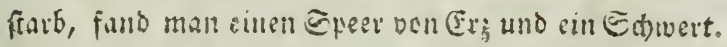

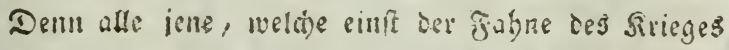

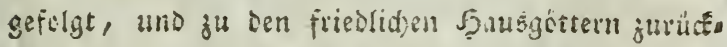

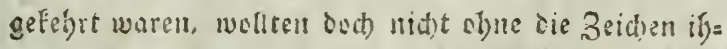

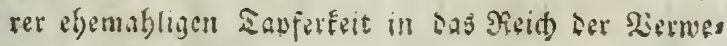
fung binúber seben, 


\section{$-78-$}

Bey ten Fömetn und Bjriedten war es allgemein angenummen, alles, was bem Serfturbenen im Seben zum Eobe gereidht hatte, voer was nach feinem Şins fideiden ifm Na(t)rubm zulid)ern Eontre, auf tem Sd)cterbaufen zu verbiennen, zugleid) mit feiner afde in oer Ulrne aufiuberwabren, und fo in ben Ed)ocs der Erde zu verienten. Iese GienerErene, ieder Sorbeer, Den er im Reben erfämpft hatte, wurs de ifin in das Girab mitgegeben. Der Smberater, Der Şeerführet, Der Briumphator, Der Sonful, Der Bürger und der Soldat wurben alle mit jenem pe beerbigt, der ifrer 2 sulrbe gejiemte.

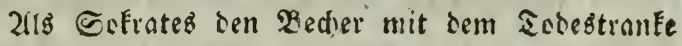
geleert hatte, wiffelte ibn einer feiner edulter in etnell Mantel ein, Damit ber Brếfe der Ffitefos

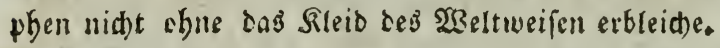

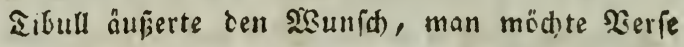
in fane llrue werfen. So bewiefen bie Menfden immer auch im Fude nod) ihre 2lubänalidfeeit an bas, was ifnen in Reben theuer gervefen war; uno fino sie beutigen Menid)eu anders? Diefelbr Eitte herridts te in ben Sulonien. Die Fenerlidfeiten ben den $\mathfrak{R} e$ = denbenängnifien waren in ben Frevingrtästen uns Solenien nad) Dem alter, (Bsefollechte und @tande der Serfforbenen eben fo verfdreden. Rilien, Sisien uns andre Blumen bedeffien ber Sinder und Mäbd)en Gräber; Şalşilerden, 2lrmbünder, Seaarid)muf́, Eolt= bure Salben legte man in sie Grabmabler Der frauen; 


\section{- $79-$}

Der Mann rufte bey feinem Edfwerte, feinem Edfit= de und feiner \&anje.

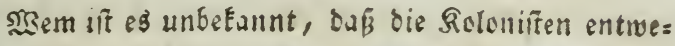
ber Geldaten warell, soer'ven Eoldaten berîtamina ten? Die Dišmer námlith ffiegten, ifre Beteranen ster jene, bie fid) Iurd) Surferfeit ausigezeid)net hat: ten, in bie Sactonien ju futifen, uno ifnen Bitund: ftucfe in ben Sänsern, bie fie ercbert hutten, anjus werfen, theils um lie füt iffe Sbaten gुu belohnen,

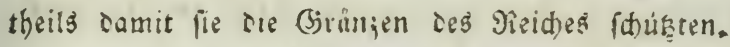
Da jie sies in allen Fistenien thaten, fo bectadteten fie es gewis auch in ten entferntefen PJrovinjen, Dis am Fifeine doer Der Donau Jagen. *)

Finen andern $\mathfrak{B} e$ eweip liefern ung bie in ben $\mathcal{S}_{0}=$ Ienien geíhlagenen Nünjen, worauf man nid,t ielten Den $2101 e r$ ser Eegienen, Faffnen unb andre firiegs: geiten finter. Diefelben Beichen Eonımen hăüir auf

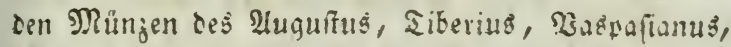
Eevtimius Eeverus, Caracalla, Seliegaba'us uno

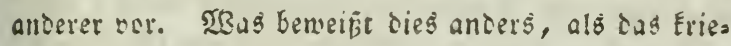

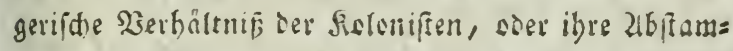
mung son Suriegem? -

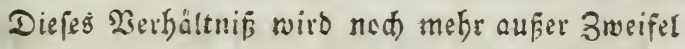

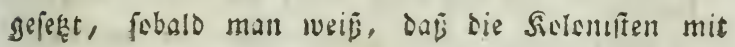

") 2"lae Sedes erit emeritis, quae rura dabuntur, Quae nuster veteranus aret, qquae moenia fessis? Lucanus' $\mathbf{s}, 3+4$. 


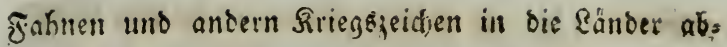
gefubrt wurten, meru thren das Biefes befikungen

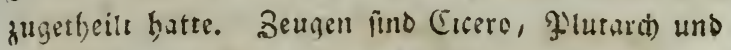
21Fwarbus. Flutard fubrt ten Umitant, daßj ous Saprtitum der Solonie, die rad Sarthago gefüfret werben iclate, ierbrad, als eine bofe Sorbeceutunis an. Elcero fitid) an mebrem Stellen, wo von den

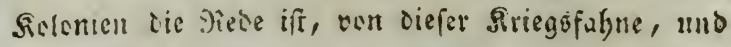

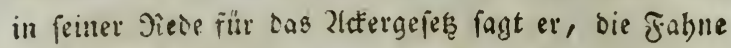

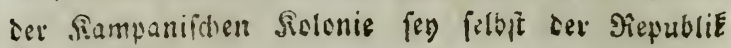
furdibar geverdent.

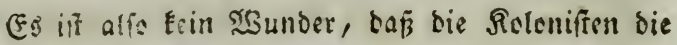
Eriencriden Gebräudte uno Eitten berbebalten bas ben, uno mit ifren Saffen fino begraben worten. Inteffen folgt caraus niđjt, baf man bie Giäber tee Siolenifên ven ben (joräbern ter Eoldaten nid)t untere fadeloen Eöme: fobuts die affde ser einjeluen in Uts nen aufbemahrt ift; fo Fann man mit $\mathfrak{S}_{3} a$ frheit bes

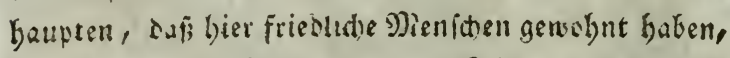
wenn man aud) Sisaffen hey oen Seiden findet; und nur bag Eann man mit Brunte für cin Grab vols Srregern halten, worin alles sbue Dromung auf ein= anter gelbäuft ift.

Hebrigens läjit fich aud reidht etElanten, warum einige mit, antere sbme SOSaffen begraben wutden; tenm nid)t alle kelomiten framnten ven Soldater her, viele waren ven birigerlicher 2bEunf. Darum wutben in oen Stolonien 2Rungen mit verid)tedenem 
Etåmpel ausgeprågt; auf biefen fielft man eines Sö loniffen, ber Ddfen vor fid ber treibt, auf ben ans oern trågt er Siriegesjaetchen; iene waren alfo Bărger, biefe $\mathfrak{B e t e r a n e n . ~ M a n ~ E e n n t ~ e i n e ~ M u ̈ n g e , ~ d i e ~ R i t u s ~}$

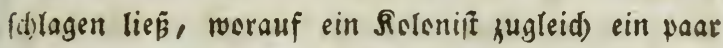

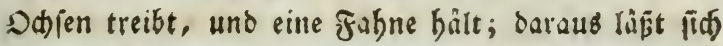
jiemlid, Elar folgern, baß̈ Bürger aus ber Stadt, Die Soloniffen aber aus ben Seteranen gewählt uno in bieje Folonie geidifit worten find. So veridies een nun bie ?l6Eunft der Soleniffen war, fo verfhie ben waren aud) ibre (J) brâuche bey \&eiḑenbegängnifien.

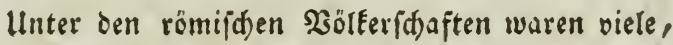
bie ibre Icoten verbrunnten, viele bie fie zul Eroe beffatteten; und fo nabmen die Rolsniften, audb bies fen eder ienen (jebraud) an, nacbiem lie von diefen cter jenell abftammten. Sielleidft war es̉ aber autí)

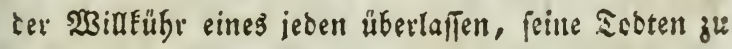
verbrennen oder zu beerbigen.

Doch genug bievon. (Es ift bintanglid bewiefents

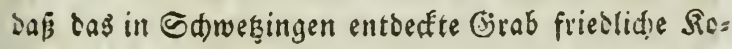
Ioniften uno Eeine Sirieger in feinem Swacke bewahk = te. Şier hatte alfo eine rëmilathe Salonie fich anges fiede!t, das̉ bezeugen die gefundenen Bäder unb Birat.

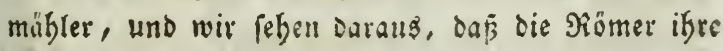

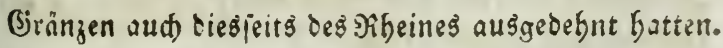
Nie würben fie in unjern Bjegenden Bä̀er, Giräbe: soer andere Monumente des friedens erriditet haber, wenn lie nidht in rubigem Beplese aeweien wâtera. 


\section{$-82-$}

zittảre, Zempel, Bifoniffe ber Sëtter unb antere heilige Dentmäbler wurben wobl an ber feindlithen Bränzc, bod niemals sin Brabimalgl çefunten. Die 9iö:

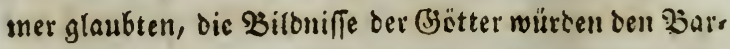
baren einen beiligen Sd)recten cinprongen mo fie jus

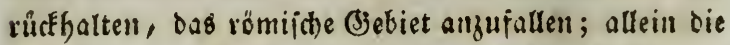
teberrefte dar Berfturbenen fez̨ten fie nie der befahg

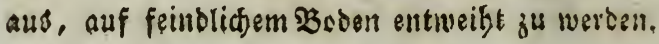

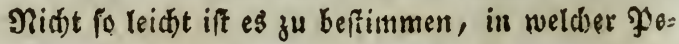
victe rọmijhe Sioloniffen bier gुewebnt baben. Mehs tere bebaupten, fdon im jueyten Sabrhunderte fen ber Bjebraud), bie Fobtell zll verbrennen, abgefem: tmen; bas in Sd)nessingen entbecfte Brabmahl fönt: te alfo "nad biefer D)?einung ins erffe poer guvelute Sabrfumbert verfegt werden. Sllbefien beneifet uns ₹sabrattus, baj nod im britten Jabrbunderte bas Serfrennen üblid) war; eben bies aeigt Sdefflin in feiner Beidreibumg dẹ đrräber, bie in ien Mibeinlane ben fiito entoefft worden, Boofried bemelét bey, dem

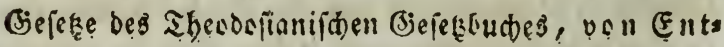
weibung der (oräber, bap defer (jebrauch an Ende bes vierten Gabrbunderta, uno bia zu Shecte.

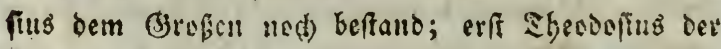

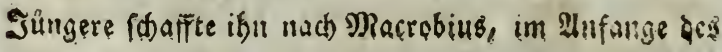
fünften Jabrgunderte\$ $a b$.

2lus einer fo zweiferlyaften Sache infen fïi) alfa

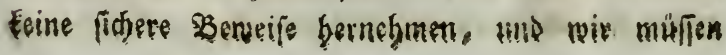




\section{$-83-$}

Dem alter unferer Dentmäbler anderwät nafj: forident.

In Sd)weteingen fowobl, als in den übrigets Mentumenten hat man Miấngen gefunden, welde in bie Zeiten Irajaut, Zutuning des 2̧seifen und peinet

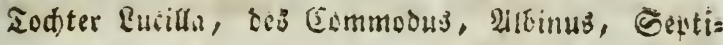
mius Geverus und des Caracalla gefförent. Edjon unter Eraian alfo batten fid) bit Siomer bier am Mikeine uno Necfer niebsrgetaffen; uno bis zu Caras

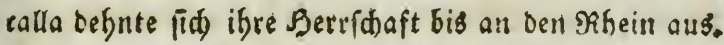
Facitub, ber unter Rrajan lebte, fagt uns, bas

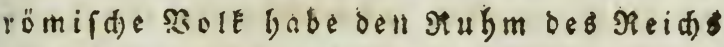

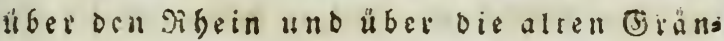
jen verbreitet. Dief paßjt auf Eeine Bizgento beffet, als auf bie unfrige zwifhen bem Mibeine, Necter unt Main, cher auf bie ganze Sandidaft

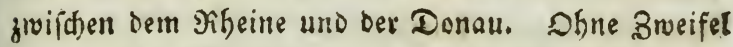

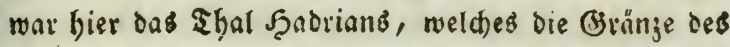
Reides Eeffimmte. Epener befauntet znar, bereits unter den 2ut toninen bate bas römiid) Wieid) fid nid)t

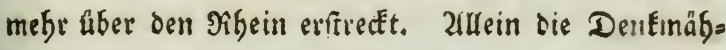
ler bie unter Caracalla, Şetiogabalus und Zllerander Ëeverus anf biejen llfern find erridtet worben, unb felbft bie @tadt 23 adell, die von dem Znteninus 2lureliug, fonft Caracalla genannt, ben Namen $2(u=$ relia erfielt, verfünden Elar und unwiberipredblif,

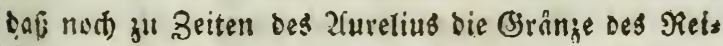
d)e unverlegęt beftanto, wir lie Şabrian befimm 


\section{- $84=$}

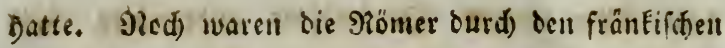

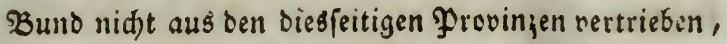

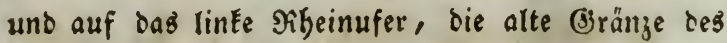
Etaates, jurülf́gedrängt. Erift fpäter fubblten die Deutiden ifre Siaft, riflen \&änber von bem Sieiche $a b$, und gründeten auf bie Trủmmer beffelben bas Reid) Der Zranken.

Şir beffen, unfern lefern wirb biefe $2(6$ fd)weio fung nifft unangenebm gewejen lenn; gern bebt ber MRenfd dell Sdjleyer, ber auf ber Ge(d)idte ber Soczeit liegt, und gelingt es iffm, einen Blikf in Dies Dunfel zu thun, fo Durdfftrömt ifn eine idjaue:=

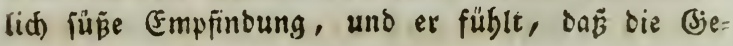
fabid)te uns Rebenşweişbeit lefrt.

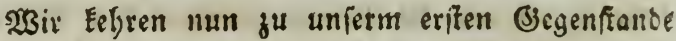

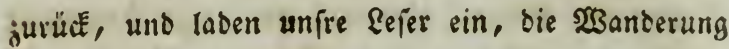
burth ben Barten fertżufeşen.

Diefem Monumente gegenüber freht ein anberes

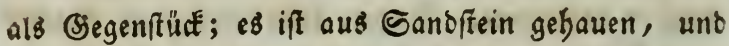
bietet bem Befidauer bie 2fttributen ber Bartens. Eunft bar.

24uf ber Şorberfeite freft folgende Intidrift:

Miraris

Quisquis obambulas

Stupet ipsa

Quae negaverat

Magna rerum mateT

Natura. 
2(uf ber Sillefieite lief't man:

Carolus Theodorus

Deponendis curis
Sibi Suisque
Ludendo fecit
Monim. h. p.
MDCCLXXI.

Şir wollen es nidte verfudten, Sicie Jnid)riften, bejonters jene auf Eer Borberjeite, zu redtfertigen;

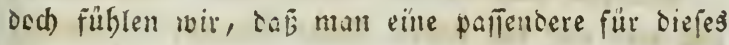
Giegenftút zu sem intereffanten Monumente gegen ulker bătte finden Fönnen.

Diele Zinlage fomobl, als bie, weldide ienfeits Der grểen 2utree liegt, unb auf bem fllane ebenfalls mit A A bejeidnet ift, bieten in ifrer Eintheilung übrigens nidts befonteres sar. Das Banje ift ein

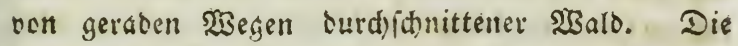
3åume inbefien funo ftarÉ uno präd)tig; verídiedene (3)ruppen von Sotftannen uno Eerden überraidjen burd) Sdönheit uno idywelgerifichen $23 u$ ubs; viele

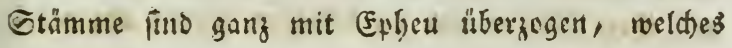
Dem Banjen einen romantifden CGarater gibt; vor:

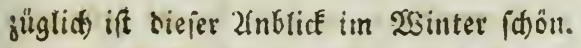

In biefer gartbie, fo wie im gangen Giartens berwunbert man den üppigen $\mathfrak{3 u c t s ~ b e r ~ S a ̈ u m e ~ u n e ~}$

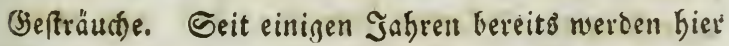
Eleine Sdläge in Dunkelbiebe gebauen, wo matt

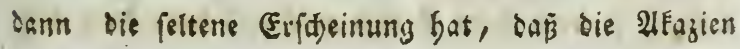




\section{$-86-$}

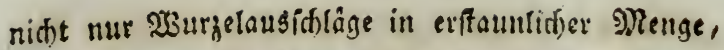
forroen fogar auf pedses und orenfigiăbrigen Etüm. inen in erifen Sommer Stocfausjhläge ven gueli uno mefir Sdub treitell.

Son Sunftwerken fintet man bier blos in sen (3)ruppen von Nabelfolze, um bas mittlere Doal, vier Urnen von Gandifeine, mit attegorien in halb erbabener 2lrbeit, die åußerft föbene forman baten.

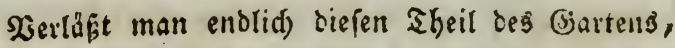
unt tritt auf ber linten Seite Geraus, fo befintet

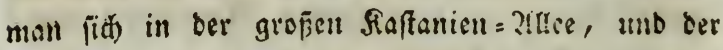

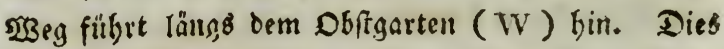
ift eine Offtbaumidule, weldhe auf befefl bes Giros. berjoges angelegt wait, und im Sertife biejes Jabs= res vollenbet fernu wirb. Diefer Dbirgarten entbält actet unb einen balben 2 orgen, worauf bie vortref: lidffen Dbiftarten bereits in Ueberfluffe angepflangt find,

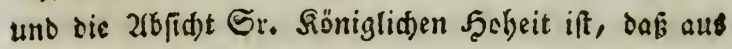

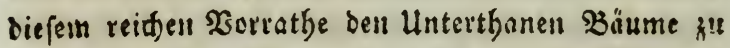
Şepflanjung der Strap̄en, Der bürgerlid)en Ilimente

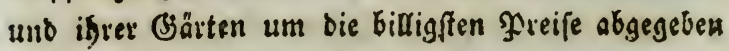
werden follen.

Diefe Obftbaumidiule entbălt bereits 165,000

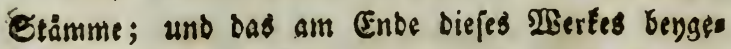
fügte \$erzeiळnip ber verfobiedenen im Edwegingert angepflangten Dbftarten beweibt, baf man diefert Zlweig ber Gavten = Rultur nidjt nur nidt cernnd)

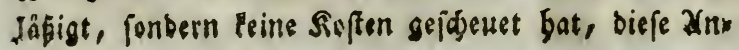




\section{- $87-$}

lage auf ben böbffen Grab der Sorfemmenbeit ju bringen. Săbrlid) wird fie nod erweitert uno vers mefret; Darnber bat Der Grostberzog feinen \$Billen befiumnt ausigefpredent.

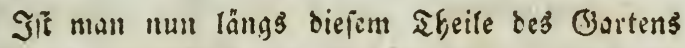
surch die grese Saftanien. aftee fortyegangen, po bee

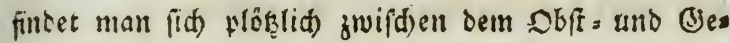
müpegartill auf einem Funte, wo man auf alten

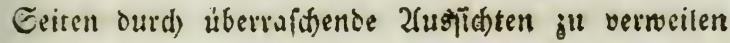
eingelaben wird. Heberall verliert fid bas 2fuge in

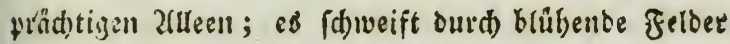

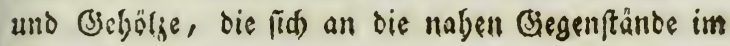
(jartenl lietlid) antreigen.

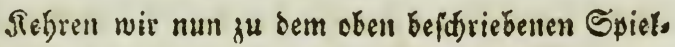

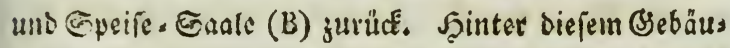
be fitten wil mod) eine fleine englijde anlage, morin

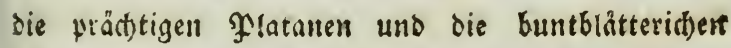
athorne bewanbert zu werben verbienen. Eir juglans angusti folia Ait. wirt ebenfalls bie 2ufmerEfamfeit res Sismers und Jreundes ber Siaturigeftichte auf fid) jichen: bisfer Baum bat am Stamme fünf

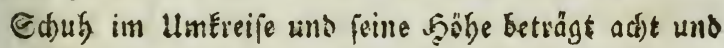

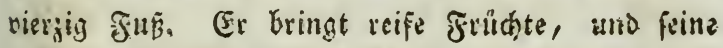
bellghtimen, gefieberten Brâtter inadjen eine cuffers urbentlid fobine S⿱乛龰sirturtg. Man findot bier fertuer einen Crataegus aria Suecica, bev fünf Canth juey

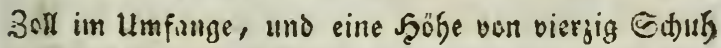

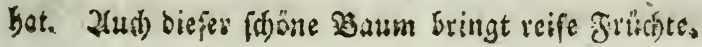




\section{- $88-$}

2(ud) ein Sunftwerk ift in biefer Fartbie "nod ju betrad)ten; es if eine (Bruppe von Iritonent mit Sees If ferben (E), welde şaffer fuenen. Das Gjanze ift voll Strin und oortreflich gearbeitet, oer Meifter aber if unl:tannt. Dies Siunfinert if ebenfalls aus Der Serlaffenfaftaft bes Söniges Stanišlaus in Rünes ville geFauft und bieber gebrad)t worben.

Der Biemüjegarten (V) iif mit mefreren Mauern Dur(t)fonitten, woran die Ffirfid)e als Bivergbăume gegugen weroen.

Die Ireiberey (U) ift jicmlich beträt)tlid, unt vie Gras: : und Ireibbäujer entbalten Sdäg̨e ven fel:

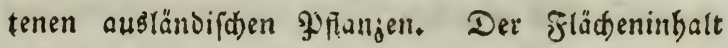
biefer Inlage beträgt fed)s und oren viertel Mlorgen.

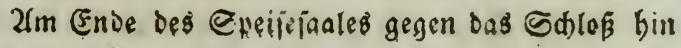
fteht eine Ulmie (Ulmus Sativa du Rai) wel(i)e na:t)

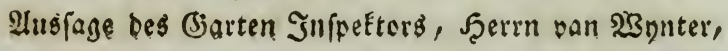
im Sabre I 762 gepflungt wurbe, uns ję̧t viergebn Sobuh, fiebell 3oll im llmfange bat, uno fed)s uno neungrig Ju(a. bad) ift; cberfläd)(id) bered)net, wïrde iie orey uno ein balbes Slafter Sycl; geben. Dev

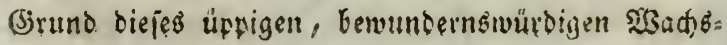
thumes if ebenfulls nut in bem Şorizonta!naller ju fuchen, welches uberbaupt in bem Sdyweşinger (jar. ten bie S?atur els 23 irEmittel Des aus̄erordentliden Fflanjenwuthjes anjuwenben (d)eint; benn aud Gier, po biefe Ulme prangt, ift bas (Erbieid) fandig, unb f(beint niकts weniger als oer segetation günftig zu 
fentt. Eo entfant int cinem Beitraume von brey bis vier uno fühtsig Sabrent auf einer Sandpläbs von

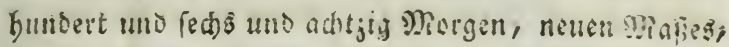

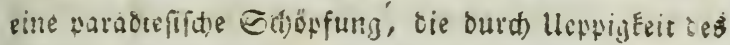

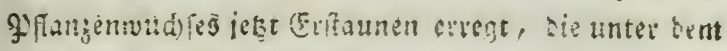

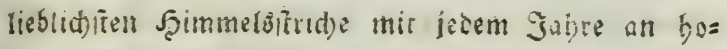

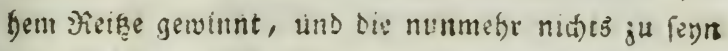

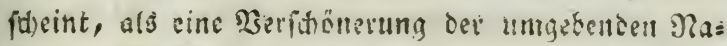
turicenen. Der Frembe weilet geen in Diejen cuften=

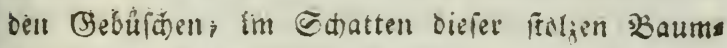
gânge, key bem Befhauen der therall anfigeffellten

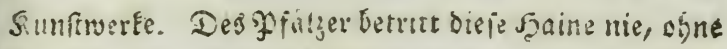

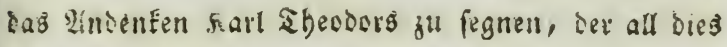
Edjöne uno (jirége geid)affen bat, und obue feinem

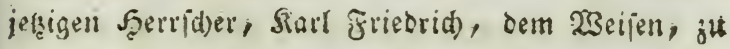

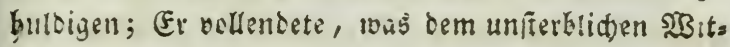

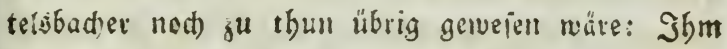
vertanket man cie Bseförcerung des Baumifulmejeng, bejinvers der SGitbrumiäule, voll ber wir bereits

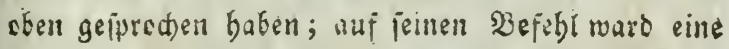
erotifhe Baumithule angelegt, bie in Znjefung

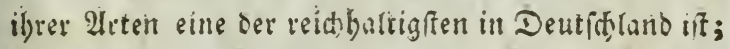

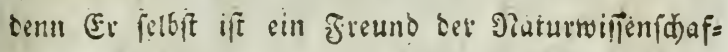
ten, und füblt, weidge :ortbeile das Stucium ber:

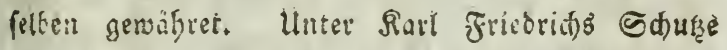
waro bie ebemafiglige Drangerie ju einem 2frboretum angetegt. Damaļ war das berủgmte gorftinfitut

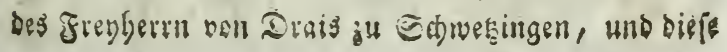




\section{$-90-$}

weue Infage wato alfo ju einem forft, botanifthen Barten beffimmt; bres Snftitut beffeht nun ; war nidft mebr in Sdbweşingen, alfein beffen ungeadtet ift biejer gerfit =botanifhe Garten ben in Şeicelberg ftubierenden jungen Forfmánnern ftets offen, und jie Eännen bier bie ju ifren Grubien nổthingen Exems plare ofne Inffand erbaltent. Săbrlid) werden ferner bedeutende Summen verwendet, bie (3)ab = und Ireibs bauşplanz̧en żu vermebren, bie entweier mit barem

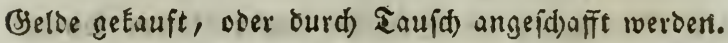
So verfanumet biefer erbabene färft ben ben ungünftigs ffen Beiten nichts, was zur Befördertang der Şünfte uno

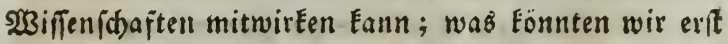
erwarten, wenn Frieben bic Menjidgeit beglủette? -

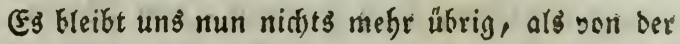
grof̧en $23 a$ fermajhine $(N)$, weven die Fontänen de Bartens ifr $\mathfrak{3 a f f e r}$ erfalten, nuch etroas ju jagen.

Der Dberbaubirekter uen Pigage legte im Jabre I762 oas elfte Warterwert an, weldes aber bles aus einem von bem fogenanntent Scimbad)e getriefes wen Sdjöpfrade beffant, und das $23 a$ fer nur ju einer Şóbe von fünf unb zwangig ₹ū bradtete.

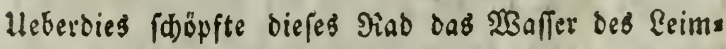

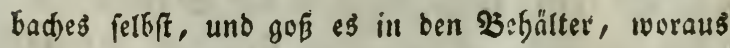
es bann burch Ieid)el in bie Fontannell geleitet ward; ba aber Daß 2 aller biefes Baches faft immer trübe ift, und fefr vielen Sd)lamm abjeset, baburd) bie

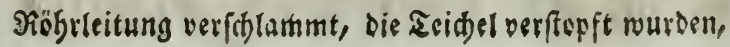




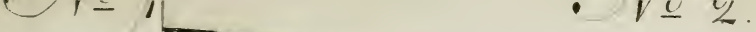

[n]
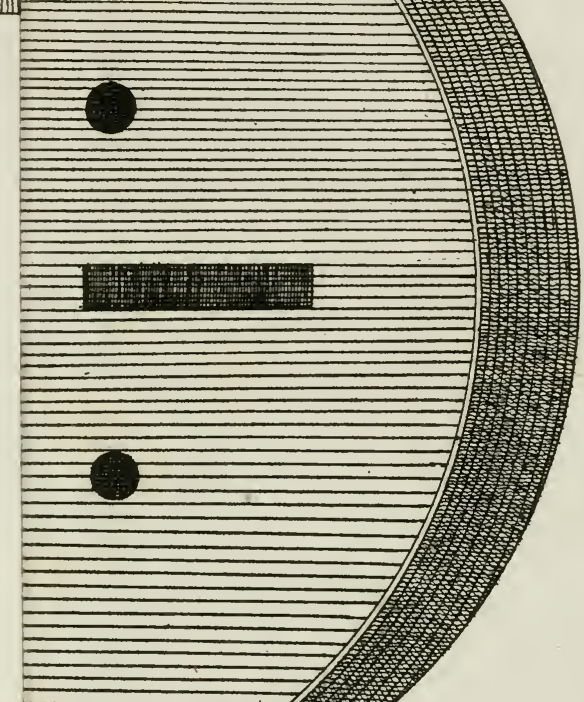


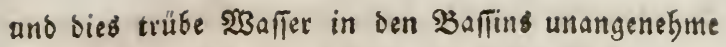
Dünfte verbreitete: fo wurbe befhleffen, eine zwar ungleid) Eoftbarere, aber weit grwecf́måzigere uno grös fiere $\mathfrak{B}$ afferteitung anz̧ulegen. Der Brunnenmeiffer Jobann Breyer mit feinem Sobne, und Gobann Pfrang waren bie Männer, welden man biefes WुerÉ übertrug.

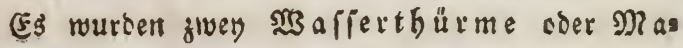
fdinenbåufer, gebaut, und zwar zuerft der \$lurm an bem Reimbade, bie untere Rafdine genaunt, auserbalb bes (5artens nidjt ferne von bee

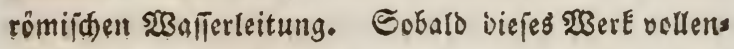
Det unb feine शrsirfung erprobet war, wurbe bie ziveys te Rafdinerie in ber Nabe des Sdlolles, bie obere Na a fille genannt, angelegt. Diejer zuvente $\mathfrak{B a}_{\text {a }}$ fertburm ift in einem einfachen Style über bell ans dem linfen Früget bes Salofles vorbeyfließ̈enden Reimbad) erbauet, unb gefänt bem 2(uge ourd bie

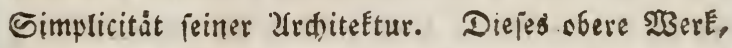

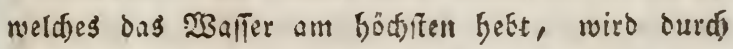

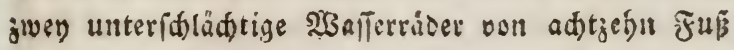
im Durdmeter, und ein Fus, fünf 3oll Sdjaufel= breite, in einem geidtollenen Cjerïnne von drey Fü Fall, und einem 23 Buffertande auf berm fabbaume

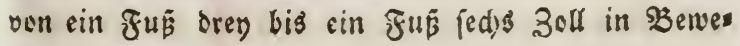
gung gejest.

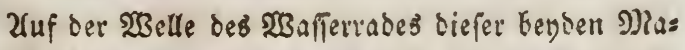

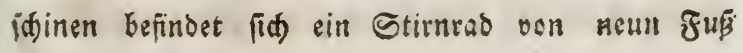




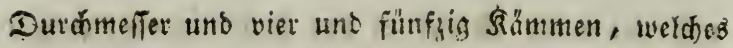

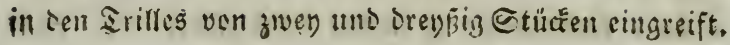

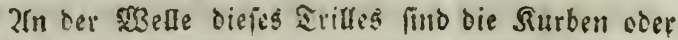
Siruminjalfen a neun Soll $\mathfrak{h e d}$, und benegen aljo

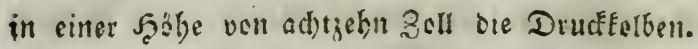

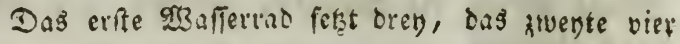
Druffumpen in Bewentung; de Etiefal fint ven gegolfentem Mrefing, Dir Durdineffer ifrer innem

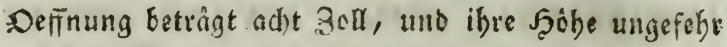

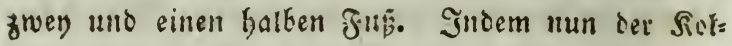
ben b in bem Stiefel c ourd) den Srummjapfert a

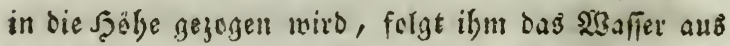
bem 3 brumen surd) die êngrobre $e$ in den Eties fel c burd ben Druck ber "äuern Suft nach, unt fülut foldhen voll Şaffer. Sinte nun ber Sorben b

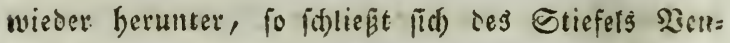

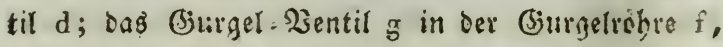

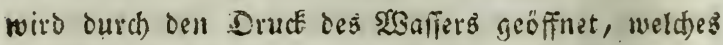

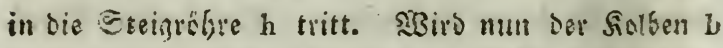

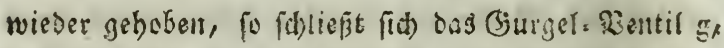
und der Stiefol c miro von Seruen ourd dus Bente

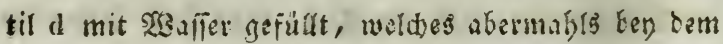

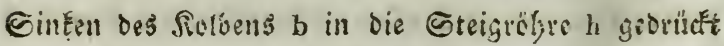
wird. Da nun diefe Bewegung ber Rerbenirange

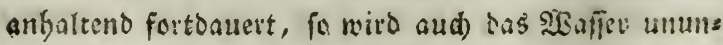

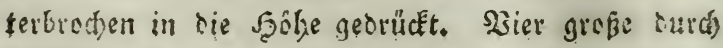

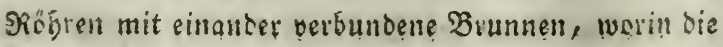

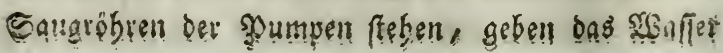


in biefen fieben Druafpumpen, die es nun bura bren Bleyerne Stehrabren, Deren iede im immeren Durd)= meffer srey 3all hat, die diffe des Bleyes aber einent

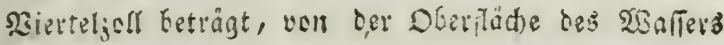
bis in die grofen șanerbebälter, alio zu einer sabje von huniert fün uno oreyäing Fü baben.

Diefe Brumnen find nod) nie ausgevumpt rorten, und durch bieje fieken Pumpen werden in einem Zeits yaume ven jwölf Stunden fün fundert und neunjitn

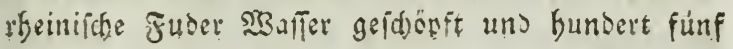

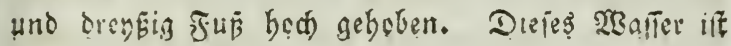

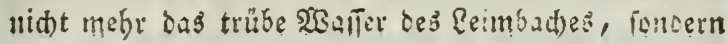

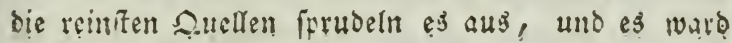
ftets Eay ber Safel fiarl Sheovors getrumen.

2tuf tem Shume, in einer Şöe oon füf und funting Juf l̈ber sim Boben, befinden fid) ziwey RBaffetbet,alter ren farken forumpenbley, welde shs fammen fieben und neunig rheinilitse Futer șaller falfen. Die ssante derferben werten rurch Gieriple טon eichenen Salfen, die anverthatb FuE oon eman rev fteben zufammen gebarten. Der eme ciejer bes Gäter wiegt vier uno adtzig Bentnes, mo bivoe Find ourch) siobren, wie cie Fumpen, verbuntert.

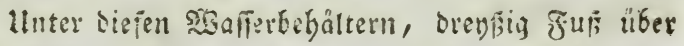

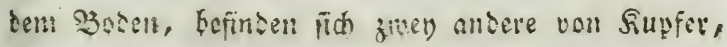

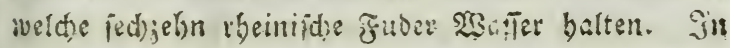

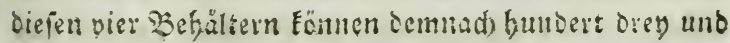

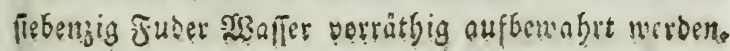




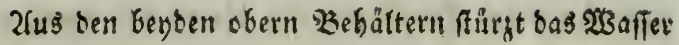

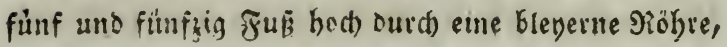
bie Eructrobre genannt, berunter. Diefe פiobre if trichurformig uno you farkem Blen gemacht; ifre obere Deffmuna betrảgt zwey und einen balben 5 (b)

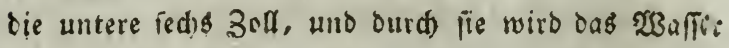
in ven groben sallin geleitet, uno bewiret baun ben bëdiften Eprung.

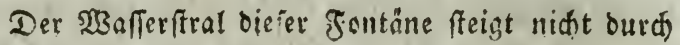
eine einţige runde Deffnung emre:, fondern die obere Coleibe bat lảnglitbe uno Elcine runbe Deffinungen; man jebe auf oer Supfertofel die 26birlonng No. 2.,

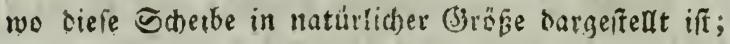
burth otefe fo ourchlócherte Saberbe fpringen mebrere

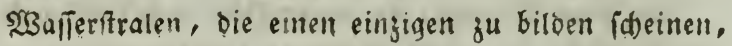

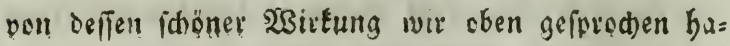
ben, uno beffen ausfwringende $\mathfrak{S}_{3}$ affermaffe in jwölf Gtunden buncert uno fünfaig Fuder beträgt.

Die vier Elẹineren Fontänen in bielem grefien Bafin, fo wie dre vier Epringtarumen des oyalen Baffins erbalten ibr $\mathfrak{W a f f e r}$ aus den zwey Eupfernen Şafferbebåltern, weldbe nut oreyß̈ig Fuß $\$ 30$ en fieben.

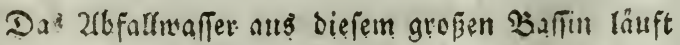
ju bem fogenamiten Seepferbe binter bem Epeifejale

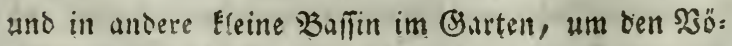

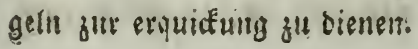




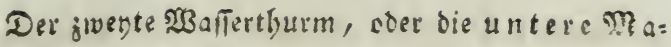

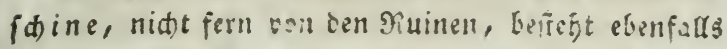
aus jrey Wafjerwerken mit iemjetben 3iace uns Sumpenwerke, wie in ber sbern Majditre; Dech find bier nut orey grofe, surth roblen mit einanter ver. buntene Brunnen, bie ebenfalls nah tie lino ausaes pumpt werden. In biefem Sfurme fino nur iney Bebălter von Stey, deren einer fieben, dex andere

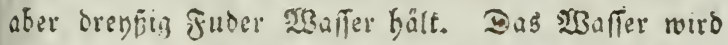
bier von Der Dberfläd) adst uno fectiia fus bed ge= boben, bat bis jur ebenen (5rde einen Fall ven orey und viergig Fü und flieft Dann ourd) bleverne Sich: ten unter bet Fide in ben Barten.

In trualf Etunsen werben ourdo siefe groey शia:

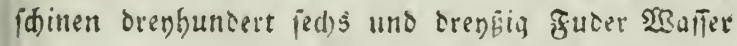
ju Der fäbe von trey uno viergig frü gebaben, nels

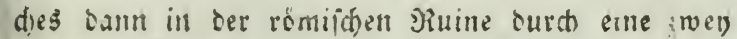

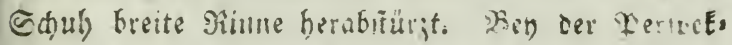
tive trånfelt es in Der Gratte berab; bey bem Degels Buffin fweyen es̀ brapiefn Sigel mit Lem in ber MJitte berab; am Tempal 21pollons bircet es die Entegels Eaśade, uno wito uan oa an veríchietene Dite im

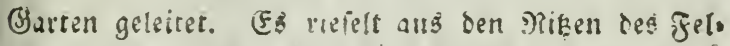
pens, worauf Fan likst, in bas Becten unten ain Suże, und bildet ferner Sajelbit die jwey Elentent Fontånen.

In zrwölf Stuncen merben baber burd die jwery

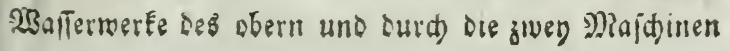


Des untern 2 Gurmes adthundert fünf und fünfaid

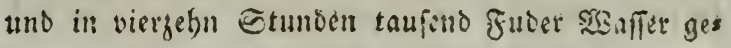
boben, uno in bie fontüttent bes Gjartens geleitet.

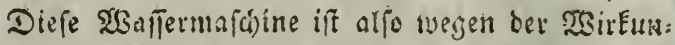
gen, bie fie bervorbringt unt wegen ifrer einfacten

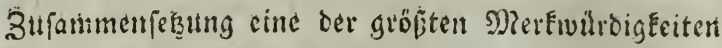
in bem Sodreringer Barten, who wit glaubten um to inebre eine Eleine befchreibung savon liefern zull millifen, da biefer MieifferwerE nid)t fo allyemein ges fannt if, alo es gekannt ğu feign verticnt.

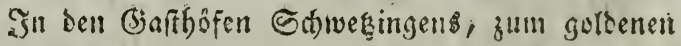

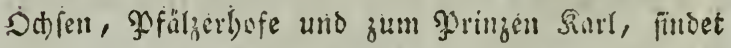
Ser firemce Mienfden, welde bereit fitto, ifn in ben Barten jil begleiten, thito an jebcm merEwüroigen

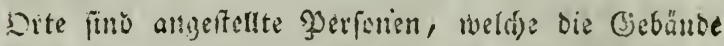

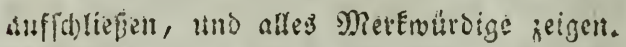

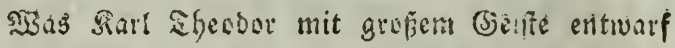
tint aușfübrte, werten bie 2ibEommlinge ber etlauch= ten Bülytinger nid)t unvellendet lafien.

Sart Friedridh Gat feit tem ZeitpunEte, wo er bie Siegiermis Diefes fobönen Ean es ükernabin fidon for viel gethan; Situfen wir alio nid)t mit 3uverides bof:

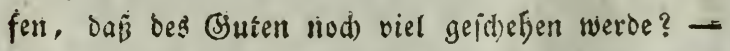

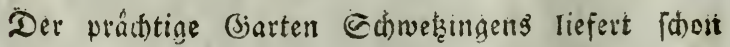

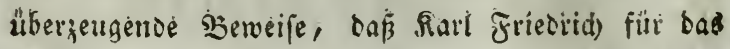

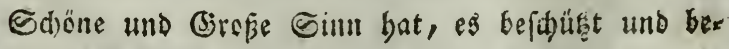
füroert. 


\section{Fเป็่นเที}

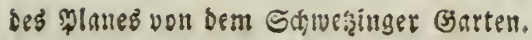

A. Daz Gd)loß. Elehe in ost Sefureibumis Estte 4

I?

B. Der Epeife =unb Epieliail

C. Das̉ Zirtel: Drangeriehauz

D. Das Romórientaus

$=$

$=$

E. Das Epefierb

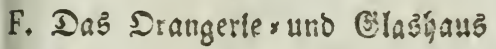

G. Der Iempel Alpallonz

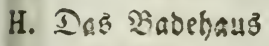

I. Die Peripettive

K. Der Tempel cer Sootanif

L. Wafferleitung

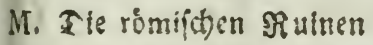

N. Die 2 saffermaidiene

0. Saperne. OO. Baummagajin

P. Ser Tempel Marturs

Q. Die Drehbrúce

R. Die Moid)e

\section{$s$}

T. Der (5ott \$an auj einem Feljen

U. Treibs und SilaẺbåujer

W. Der SGifgarten

X. כaร 21rboretum

Y. Die Mutterichule

Z. Die ミిaumi்ule 
a. Die grof̣e fontåue: Irton aufeinem Delphin

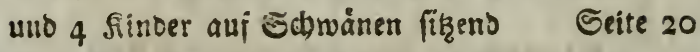
b. Bier fleine fontinen, ebenfallz Rinber auf Sdowånen

c. Bier Hinen von Marmor

d. Eine Fontáne mit Siucern auf Dradsen ebent.

e. Sranacileplats

f. Blar fur cinen botanifdsen Garten beftimmte Bieredte

?

g. Der englifiche (jarten

h. Ehinefifide srude

i. Der Rhein uno dis Donau; Foloffale Statuen

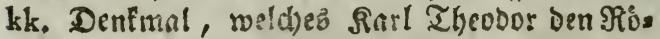
mern und Deutiajen errichtere, beren \&eid. name und Utren hier gefunben worben Das Ėegenfúct bierzu

1. Die marmornen SBiften Alexanberz, פittyri Datesె, Şaorianz uno Domitlanz m. Minerva

n. Igrippina $\}$ aủ Marmor

o. Mertur

p. Die Folofiale Şirfdogruppe:

q. Die vier Elemente

r. Dab Daffin mit $\mathfrak{W}$ aff

s. Bacbanalien bon finbern

t. พad)ub

u. Sialatbee

v. Pallaz

w. Iuftitia

x. Bier Uraen, morauf oie vler Beitalter vor. geftellt fino

AA. SPartbleen im framzoliidon Ciejdgmade
23

20

41

58

59

60

6 I 84

48

47

44

33

32

26

24

-

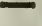

$-$ 



\title{
Verzeichn ifs
}

\author{
ธämmtlicher
}

Bäume, Glas - und Treibhauspflanzen

$$
\text { d es }
$$

Schwezinger - Gartens. 
$a+b$, arboretum.

f. frey Iand.

g. Glashaufs.

w. Treibhaufs,

* müffen über Winter bedekt Werden.

Die' Abbreviaturen der Autoren kennt jeder Bo. taniker, daher die näheren Erklärungen derfelben als überflüfsig weggelafsen wurden. 
A broma augufta $w$. Acal y pha virgara $w$. cuspidsta W.

Acer tataricum arb. pfeudo platanus platanoides fol variegatis

$\alpha$ laciniatum

$\beta$ fol. varis.

rubrum

$$
\text { mas. Horto par. }
$$

dascyrarpon. Ehrh.

faccharinum

palmatum Thunb.

ftriatum du Roi

ponfylvanicum

fpicatum Lam.

Opalus Lam.

campeftre

fol, varieg.

opulifolium Willd.

monfpefsulanum

creticum

Negundo

hybridum $W . C$ at.

Achyranthes argentea w.

A corus gramineus g.

Adelia Acidotong.

fpinofa g. 
Adiantum Capillus veneris w. pedatum g. $f$.

Aesculus Hippocastanum arb. fol. arg. varieg. fol, aur. varieg. Pavia a rubra Aiton,

$\beta$ fpicata

lutea Willd. macroftachys. Mich.

Ageratum verbascifolium w. Agave Veracrux Mill. g. americana $g$.

Albuca major g.

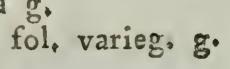

$$
\text { minor } g \text {. }
$$

Aletris fragrans $W$.

capenfis $w$.

Uvaria $g$.

Zeylanica wo

Aloé fpiralis w。

guineenfis $w_{0}$

pumila vi.

retufa w.

viscofaw.

disticha $w$.

atrovirens Perf. w.

arachnoidea. Thunb, w.

Alpinia fpicata w.

A ftragalus tragacantha arb.

Alstroemeria peregrina $g$.

$$
\text { Ligtu g. }
$$

Amaryllis Belladonna $g^{\circ}$.

$$
\begin{aligned}
& \text { adamasco g. } \\
& \text { vittata. Ait. g. } \\
& \text { ppeciofa. l'Herit. g. } \\
& \text { formolifna fo et g. }
\end{aligned}
$$




\section{$-5-$}

A in brofia peruviana. Willdog.

Amomum Zerumber w.

Amorpha fruticofa arb. pumila Mich. glabra. Desf.

Amygdalus communis arb. fol. varieg.

\section{amara.}

pumila flore pleno

\section{persicifolia,}

\section{nan?}

fibirica Lod. orientalis Ait. Perfica

$$
\text { f. pleno }
$$

Anagallis Nonelli $g$. Anagyris foetida.

Anciftrum Sanguiforba $g$.

Andromeda fpeciofa Mich, polifolia a r b.

$$
\beta \text { angurtifolia } A \text { it. }
$$

paniculata g. Ait.

racemosa $g$,

Catesbaei Willd, g. calyculata $\mathrm{g}$.

$$
\alpha \text { angufifolia } g \text {. }
$$

ferratifolia, Will. Cat. pilulifera $g$. Daboecia g. oleifolia $\mathrm{g}$.

Andryal a lanata $g$. Annona triloba a $r$. * glabra arb. Anthericum fiftulofum $g$. alooides g. 
Antholyza Meriana g. xthiopica g.

Anthyllis Barba - jovis $\mathrm{g}$.

Hermannix $g$.

cytisoides $g$.

Antirrhinum Afarina $g$. trifte $g$.

Aralia fpinofa arb. nudicauiis arb. racemola t.

Arbutus Unedo g.

flore rubro $g$.

andrachne $\mathrm{g}$.

flore pleno $\mathrm{g}$.

Uva - urfi a rb.

alpina arb.

Arctotis calendulacea $g$.

cinerea $g$.
repens $g$.
aspera g. $\mathrm{gil}$.
revoluta $\mathrm{W}$ il d.
elatior $\mathrm{W}$ illd. $\mathrm{g}$.
fuperba $\mathrm{g}$.

Arduinia bispinofa w

Ar:stolochia Piftolochia g. triloba a w.

Aristotelia maqui. Lam.g.

Artemifia argentea $g$.

arborea g.

judaca g.

abrotanum a $\mathrm{rb}$.

Arum pictum w.

esculentum $w$.

fagittæfolium $w$.

divaricatum $w$. 
Aruin arboreum w. macrorshizon w. auritum w.

Arundo Bambos w.

$$
\text { Donax f. }
$$

fol, varieg. g.

A rarum eanadenfe $f$.

Asclepias Linaria Cav. W. fruticofa w. nivea w. curasfavica w. tuberofa $\mathrm{f}$. $\mathrm{g}$.

Arcyrum Crux-Andrez g. Aspalathus cretica g.

cytifoides $y$.

Asphodelus fitulofus

A fter glutinofus $g$.

capenfis $g$.

tenellus Ât. K e W. $g$, anguftifolius IV illd, g.

A thanafia trifurcata $g$.

Atragene alpina $a r b$. aufriaca. Scop. arb.

Atraphaxis undulata $g$.

Atriplex halimus arb.

* portíacoides arb.

* Aucuba japonica arb.

A ylanthus glanululofa. Ait, arb. $\Delta y \in$ y $i$ a punilla w.

Azalea procumbens a $r b$.

viscofa arb.

caleudulacea Mich. g.

riudiffora a $r b$.

$$
\text { ce altia }
$$


A2slae nudiflora $\beta$ coccinea

g!auca Ait. arb.
e rubra
canescens Mich. $g$.
fcabra g.

- Baccharis halimifolia arb:

Barleria Prionitis w. ira folia $g$.

Basella alba w.

rubra w.

cordifolia w.

Begonia humilis Ait. wo

$$
\begin{aligned}
& \text { acuminata } \mathrm{w} \text {. } \\
& \text { capenfis } \mathrm{w} \text {. } \\
& \text { dichotoma } \mathrm{w} \text {. }
\end{aligned}
$$

Berberis vulgaris arb.

$$
\begin{aligned}
& \alpha \text { enuclea } \\
& \beta \text { alba }
\end{aligned}
$$

canadenfis Mich.

cretica

fibirica

finenfis Thouin

abortiva?

humilis

Berckheya fruticora

Betula alba arb.

$$
\begin{aligned}
& \text { a pendula arb. } \\
& \text { acuminata arb. } \\
& \text { odorata Borckh. arb. } \\
& \text { populifolia du Roi arb. } \\
& \text { papyrifera. Ait. arb. } \\
& \text { excella. Aito arb. } \\
& \text { alba arb. } \\
& \text { Lenta arb. } \\
& \text { nana zrb. }
\end{aligned}
$$


Betula pumila ar $b$.

fruticofa Pall, arb.

canadenfis IV a ng. a rbo

oblonga arb.

daurica arb.

broccembergenfis $\mathrm{D}$ u $\mathbb{R}$ oi arb.

Alnus communis a $\mathrm{r}$.

glutincfa arb.

incana $A$ it arb.

ferrulata Ait. arbo

carpinifolia $a r b$.

rubra a rb.

canadenfis arb.

maritima $a r b$,

glaciofa a $b$.

laciniata arb.

Bignonia Catalpa arb.

radicans a $\mathrm{rb}$.

$$
\text { minor ar } b_{8}
$$

*3

capreolata arb.

fempervirens $g$.

pandorea Vent. g.

Bocconia frutescens w.

$$
\text { cordata W ill d, w, }
$$

Boerhaavia fcandens w.

Bomplandia geminiflora w.

Bofe a Yervemora $g$.

Bromolia Ananas w.

$$
\text { * lxvis w. }
$$

Karatas w.

Brunia fuperbag.

chryfantha Willd. w,

abrotanoides g.

lanuginofa $g$.

Bubon glabrum $g$. 


\section{$-10=$}

Bubon macedonicum $g$.

Galbanum g.

Bucco acuminata $g$.

lanuginola $g$.

obtufa $g$.

villofa $g$.

Büchnera pedunculata $g$. oppofitifolia $\mathrm{g}$.

foedita $g$.

Buddleja falicifolia $g$. falvifolia $g$. capitata Jacq. g.

Buphthalmum frutescens $g$.

fericeum $g$.

* Bupleurum fruticofum a $r$.

longुfolium $f$.

coriaceum Ait .

Burghlari a lucida w.

Büttneria cordata Cav. w.

Burus fempervirens arb.

$$
\begin{aligned}
& \text { arborenscens a } r \text { b. } \\
& \text { myrtifolia a } r \text { b. } \\
& \text { fol. aureo varieg. } \\
& \text { fol. arg. varieg. a } r b \text {. }
\end{aligned}
$$

* balearica $W i l l d$. arb.

Cacalia finutag.

Kleinia g.

ficoides $g$.

Cactus Pereskia w.

multiangu'aris w.

pendulus w.

Dpuntia w.

grandifiorus wr.

tetragonus w.

fiagelliformis g. 
Calanchoe Verea Perf. w.

Callicarpa americana

Caladium helleborifulium Willd. w.

Caltheria heterophylla $w$.

Calycanthus fluidus arb.

$$
\begin{aligned}
& \text { fertilis Walt. arb. } \\
& \text { nanus Duh. arb, arb. } \\
& \text { oblorgulus arb. }
\end{aligned}
$$

* precox Aiton, arb.

Cammelia japonica

Campanula pyramidalis $\mathrm{g}$.

Ca mphorosma monspelienfis g.

$\mathrm{Canna}$ indica

a thyrfifora w.

anguftifolia $W$ ill d, w.

gliuci w.

Capparis fpinof $\mathrm{g}$. frondola w.

Cantaria biflora w.

Caprufia indica w.

Capficum violaceum $\mathrm{w}$.

baccasum W.

finenfe w.

frutescens w.

conoideum w。

Carica Papayaw.

Carperium ahrotznoides $g$.

Carpinus Eeculus arb.

$$
\begin{aligned}
& \text { quercifolia a rb. } \\
& \text { orientalis lara, arb. } \\
& \text { Oitrya arb. } \\
& \text { americana Mich. }
\end{aligned}
$$

Carthamus coeruleus g.

ralicifolius $\mathrm{g}$.

Caffia maryland ca arb, 


\section{$-1:-$}

Caffia corymbnfi $\pi^{\circ}$.

glisidulofa $g$

Cafrine maurcena $g$.

Perazua g.

Carfyta filiformis

rppofitifolia $g$.

Ca fuarina filiformis $g$.

Cat t nanche ccrulea $f$.

Catesbæa fpinof w.

Ceanothus africanus

americanus arb.

mycrophyllus Mich, arb.

Celaftrus pyracanthus g.
buxifolius g.
fcindens a $\mathrm{r}$.

Celfia heterophylla g.

grandifiora $g$.

ftriatiflora $g$.

arcturus $g$.

Coltis aufralis a $r b$.

orientalis $a \mathrm{r} b$.

fol. varieg. ar $b$.

occidentalis a $\mathrm{rb}$.

cordata Hort. Par, a $\mathrm{r} b$.

finentis $g$.

Tournefortii $\mathrm{D} u \mathrm{~h}$. a $\mathrm{r}$.

Centaurea ragurnag.

$$
\text { cineraria } g \text {. }
$$

Cep in 2 anthus occident lis a $r$.

$$
\text { media E. ath. }
$$

Ceratonia Siliquag.

Cercis Siliquaftrum a $r b$.

canadenfis ay b.

Cereîa elegann Perfon w.

Ceftum laurifolium. 1. Hes, w. 
Ceftrum grandifinrum w. diurnum w. fæetidiffimum w. vespertinum w. paniculatum w. auriculatum Willd. W. rinctorium $\mathrm{w}$. elongatum w. pslicifolium w. - Parqui L. Her. arb.

Chamorops humilis w. Cheiranthus feneftralis $\mathrm{g}$. ß. arborescens w。 itrictus $g$.
trifis $\mathrm{g}$.

Cli alone barbata g. et $f$. campunulata g. $f$.

Chenolea difura Wild $g$. Chenopodium multifium $g$. Chionanthus virginicus ar $b$. Chiron ia frutescens $g$. linoides $g$ : baccifera $g$. latiolia

Chryfanthemum frutescens $g$.

Chryfocoma denticulata $\mathrm{g}$. Coma-aurea $g$.

Cineraria geifolia $g$.

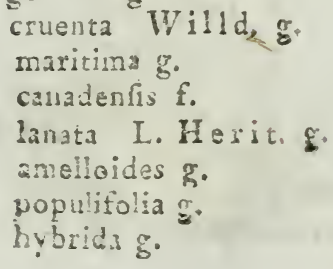


Ciffus acida w.

orientalis arb.

vitiginea w.

antarcticl $g$.

Ciftus falvifolius g.

decuratus g.

incanus $g$.

ladaniferus $g$.

rugofus $g$.

Fumana g.

mutabilis $g$.

glutinofus g.

populifolus g.

fymphitifolius $g$.

Citharexylum pentandrum $V$ ent. w.

$$
\text { quadrangulare } \mathrm{w} \text {. }
$$

\section{cinereum $\mathrm{w}$.}

Citrus medica g.

$$
\begin{aligned}
& \alpha \text { acida g. } \\
& \beta \text { cedra g. } \\
& \lambda \text { balotina g. } \\
& \delta \text { limon g. } \\
& \varepsilon \text { florentina } g .
\end{aligned}
$$

aurantium g.

\& falicifoliun $\mathrm{g}$.

$\beta$ crispum g.

$\lambda$ japonicum g.

$\delta$ plenum $\mathrm{g}$.

$\delta$ ftellatum $\mathrm{g}$.

- fulcatumg.

fol. arg. varieg. o.

fol. aur. varieg. g.

maximum

decumana g.

Claitonia Portulacaria w. 


\section{- $15-$}

Claitonia daphnefoliag.

Clematis Viticella arb.

fore rubro arb.

Viorna a r b.

$f$, pleno arb.

crispa a rb.

orientalis a rb.

virginiana arb.

Vita'ba arb.

erecta arb.

$\alpha$ elatior a r b.

intez̧riol:a $a \mathbf{r} b$.

* cirrhofa Willd. arb.

calycina. L. Herito a rb.

florida IVilld. w.

odorataw.

Clerodendrum riscofum Vent. w. infortunatum Vent w. forturatum w. fragrans w.

Clethra alnifolia arb. arborea g. incana $\mathrm{Lam} . \mathrm{g}$.

芩 acuminata (glauca) Mi i h. arb.

Cliffortia ruscifolia $g$. ilicifolia g.

ternsta g.

Clypeola maritima

Clutia pulchalla $g$.

alaternoides g.

Cneorum tricoccon, ar $b$.

Coboa fcaridens $\mathrm{C}$ a v. g.

Cof fe a arabica w.

$C$ olutea arhoiescens a r b. orientalis $\mathrm{L}$ a m. a $\mathrm{rb}$. 
Colutea Pocockii IVild a $r$ b.

Ifria? Williams, Cat. à b.

frutescens g.

perennans $g$.

Commelina africana

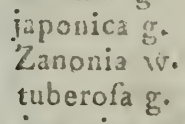

Convallaria japonica $\mathrm{W}$.

Convolvulus Cneorum

$$
\begin{aligned}
& \text { Scammonea g. } \\
& \text { grandiflorus w. } \\
& \text { Batatas w. }
\end{aligned}
$$

Conyza fordida

mollis $\mathrm{g}$.

anthelmintica $g$.

candida $g$.

faxatilis $g$.

* Coriaria myrtifolia 9 arb.

Cornus mascula a $\mathrm{b}$.

fructu flavo a $\mathbf{r} b$.

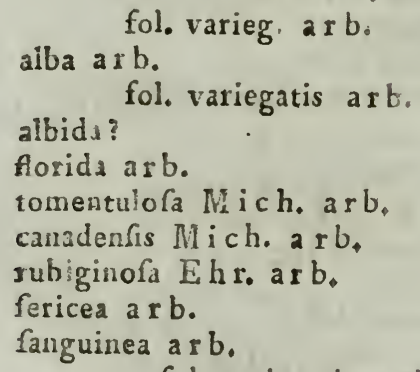
fol. varieg, $a r b$.

aiba a $r$.

- ramis cortice

obscure rubro 


\section{- $17-$}

Cornus $\beta$ ramis cortice pal ido virescente paniculata. L Herit. arb. verrucof, Lodd. a r b. punctala? arb. fuecica $f$. citrifolia? a rb.

Correa alba

Coronilla juncea $g$.

glauca $g$.

Emerus a r b. coronata arb. minima arb. valentina $\mathrm{g}$.

Corylus Avellana arb.

$$
\alpha \text { rubra arb. }
$$

Colurna a r b. americana Mich. arb. cornuta a rb. tubulofa Willd. a r b。 arborea Münchh. a r b.

Coftus arabicus w. fpeciofus w.

Cosmos bipinnata Cav. g. Cotyledon orbicularis g.

Crafsula imbricata g. paniculata g. tetragona $\mathrm{g}$. coccinea $\mathrm{g}$. pellucida $g$.

Cratagus Aria arb. 
Cratagus lucida W ang. arb.

$$
\begin{aligned}
& \text { monogyna J a c q. arb. } \\
& \text { f. pleno } \\
& \text { fl. rubro } \\
& \text { pleno } \\
& \text { fruct. flavo } \\
& \text { fol. argent. varieg. } \\
& \text { fol. aureo varieg. }
\end{aligned}
$$

Oxyacantha arb. coccinea a $\mathbf{r}$. cordata A it. arb. glandulofa $A$ it arb. punctata $A$ it. arb. Crus-galli fplendens Ait. arb. $\beta$ pyracanthifol. Ait. $\lambda$ faliciólia A it. tomentofa a $\mathrm{r} b$. $\therefore$ azarolus arb. \% fructurubroarb.

Aronia arb. pyrifolia a rb.

fructu flavo

tanacetifolia Perf. arb. dentata Hort. par. arb. caroliniana $P$ er $f$, arb. corallina a r b. prunifolia Perf. arb. axillaris Hort. par, arh. racemofa Hor to par. arb. flava A i t. arb. elliptica Ait. arb.

Crinum anguftifolium $w$. Zeylanicum g. americanum $\mathrm{g}$.

Crithmum maritimum $g$. Crotalaria capenfis jacq. g. Croton pungens $k$. 
Cunila frutescens $g$.

Cupreffus fempervirens g.

pendula W:Ildogo

difticha $a r b$.

horizontalis $\mathrm{g}$.

thyoides ar b.

lufitanica. $g$.

juniperoides $g$,

Cycas revoluta w.

Cyclamen perficum g.

Cynanchum erectum arb.

Cyperus alternitolius w.

Cypripedium Calceolus fo org:

Cytifus argenteus g.

Cajon w.

feffilifolius a $\mathbf{r}$.

nigricans a $\mathrm{r}$ b.

hirfutus ar b.

fupinus Willd arb.

auftriacus arb.

Wulgaricus arb.

glutinofus $a r b$.

purpureus arb.

Laburnum ar b.

fol. varieg. ar $b$.

elongatus ar $\mathrm{b}$.

$D$ a is cotinifolia $g$.

Daphre alpina arb.

Cneorum a r b.

Laureola a r b.

Mezereum ar $b$.

fl albo arb,

Gnidium g.

Tartonraira $g$.

collina $D u h$, arbor. $g:$ 
Uaphne pontica g. odora Ait $g$.

Datura arboreag.

* Decumaria barbara arb.

Del phin i m grandifor. A. pleno f. et $g$.

Dianthus arboreus $g$.

Dioscorea bulbifera w.

$$
\text { villofa } g \text {. }
$$

Dillenia fcandens $g$.

Diosma ericoides $g$.

longifolia $g$.

pubescens $g$.

ferratag.

rubra g.

alba $g$.

cupreffina $g$.

hirfuta g.

ciliata $g$.

villofa $g$.

imbricata $g$.

Diospyros Lotus a b.

\& fructu minore arb.

$\beta$ fructu maximo $a r b$.

virginiana $a \mathbf{r b}$.

Kaki g.

Dirca paluftris a $r$ b.

Difandra proftratag.

Dodecatheon Meadia f. et $g$.

Dolichos lignofus g.

Dracaena Draco w.

enfifolia w.

ferrea w.

Dracocephalum canarienfe.

Dracontium pertufumw.

Duranta Ellifia w.

Plumieri $w$. 


\section{$-2 I \longrightarrow$}

Ebenus cretica g.

Echites umbellsta .

Echium candicans $\mathrm{g}$.

frictum $g$.

Ehreti a tinifolia w.

Elephantopus fcaber w.

Elaeagnus orientalis arb. anguftifolia $a \mathrm{r} b$.

Empetrum nigrum arb.

Encel i a canefcens $g$.

Ephodra monoftachia ar $b$.

diftachia a r $b$.

altiffima Duh. art. $g$.

Epidendrum finenfe w.

Erica vulgaris ar $b$.

herbacea a $r$.

carnea arb.

mediterranea $g$.

aggregata $\mathrm{g}$.

parbata g.

civaricata $g$.

incana $g$.

incarnata $g$.

taxifolia $\mathrm{g}$.

cruenta $g$.

perfpicus g.

fimplicifiora $g$.

fpeciofag.

enneaphylla $g$.

amoena g.

lychnidea g.

albens $\mathrm{g}$.

fucata $\mathrm{g}$.

lanı̀ta g. 
Erica urceolaris $g$.

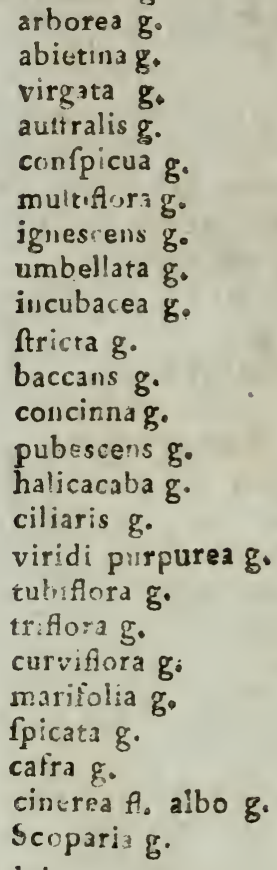

Erinus alpinus g.

Erythrina Corallodendron $w$. herbacea w.

Eucalyptus refinifera $g$.

Eucom is punctat $g$.

Eugenia unfora w.

Euphorbia Characias g.

Caput-Medurae we

ar.yedrloides g.

Evony mus europaeus a $\mathrm{r}$. fructu albo. 


\section{- $23-$}

Rvonymus europaus fol, varieg.

latifolius ar b.

* atnericanus ar $\mathrm{b}$.

verrucofus arb.

atropurpureus a $a$ b.

Fabricia laevigatag.

Eagus Caftanea arb.

$$
\text { 101. varieg. }
$$

pumila arb.

Caftanella arb. asplenifolia a r b.

Sylvatica a r b.

* ferruginea

$\beta$ atropurpurea

$\lambda$ fol. varieg.

$\delta$ creps de cog̨.

Ficus Cariga g.

minor, g.

Renjamina w.

bengalenfis w.

indica w

relingio?a w.

ferruginea $w$,

ftipulata w.

nitida w.

maxima w.

capenfis $W$,

martinicentis $\mathrm{W}$.

aufralis w.

oppofitifolia w.

Fontanesia phyllirzoides ar $b$.

Fothergilla Gardeni g.

Frankenia hirfuta gr

Franclilinia alatamaha $g$.

Fraxinus excelfior arb. 
Fraxinus excelfior fol. arg, varieg.

fol. anr. varieg.

verrucofa

jaspidea

aurea

horizontalis

fimplicifolia Will d.

americana a $\mathrm{r}$.

\section{nigra \\ alba}

fambuclfolia

juglandifolia

acuminata $\mathrm{La}$. arb.

caroliniana La $\mathrm{m}$, arb.

pubescens $\mathrm{Lam}$, arb.

epiptererea Mich. art.

Ornus arb.

rotundifolia $\mathrm{L}$ a m, arb.

lentiscifolia $H$ ort. $p a r$ a $r$ b. rotundifol. $H_{4}$. arb. monophylla a rb.

finenfis $a r b$,

nana $a r b$

quadrangularis $\mathrm{Mich}$, arb.

falicifolia ar b.

lyrata a r b.

crispa arb.

parvifolia La m. a r b,

Fritillaria regia $g$.

Fuchsia coccinea $\mathrm{g}$.

Gardenia florida w.

flore pleno $w_{0}$

Randia w.

mitis w,

Gau ra fruticosa $\mathrm{g}$. 


\section{$-25-$}

Gaultheria procumbens arb.

Georgin a purpurea lilucina coccines.

Gen ift a linifulia $g$. candicans $\mathrm{g}$. fagittalis a r b. tinctoria a r b. fibirica a r b. anglica $a r b$. germanica arb. pilosa arb. lufitanica arb. elatior Willd. arb.

Geranium et Pelargoninm.

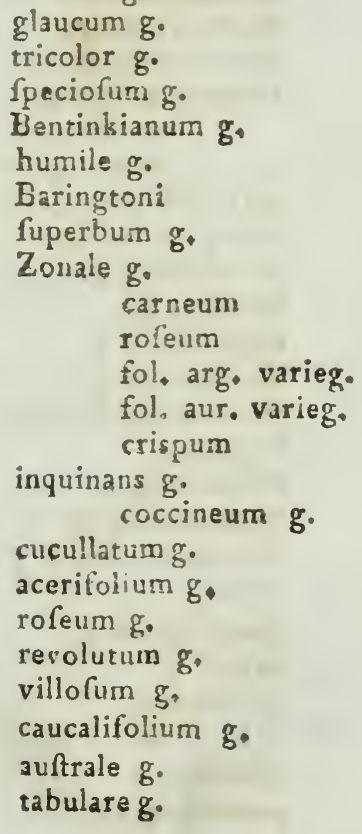




\section{$-26-$}

Geranium capitatum g.

trifolium $\mathrm{g}$.

anceps $g$

vitifolium g.

betulinum $g$.

alchemilloides $g$.

balsameuin majus $g$.

ininus

medium

Reichardi g.

cdoratiffimum $g$.

gibbofum g.

exftipulatum $g$.

pinnatifidum $g$.

cordifolium $g$.

crispum majus $\mathrm{g}$.

medium $g$.

minus $\mathrm{g}$.

cortusaefolium $\mathrm{g}$.

emargi natum $g$.

monftrofum $g$.

fcandens $\mathrm{g}$.

tenuifolium $g$.

Tormannoe g.

cynosbatifol. Willd, h. ber g.

fragile g.

ternatum $g$.

denticulatum $\mathrm{g}$.

therebintinaceum $g$.

irifte g.

quercifolium $g$.

elegans g.

acetofum g.

fol. variegatis

hybridum g.

penicellatum $g$. 


\section{- $27 \quad-$}

Geranium Cotyledonisg. grind fortim g.

incisum g.

tricuspitadum $\mathrm{g}$.

hetercgamum $g$ 。

ftenopezalum g.

Iplesicens to.

formolum $\mathrm{g}$.

anemonefolium go

incantum $g$.

tetragonim $g$.

peltatum $g$.

tomentofum odoratum g.

palmatum $\mathrm{g}$.

fillgidum $g$.

flavurn g.

alternans g.

echinatum $g$.

lobatum $g$.

hirtum $g$.

carnofum $\mathrm{g}$.

fragrans $g$.

Gesneria pulchella w.

tomentoraw.

Gingko biloba a rts.

Gladiolus cardinalis g.

Gieditsia triacanthos arb.

inermis a $r$ b.

finenfis Horr. l'ar. as b.

a crispifolia $a \mathrm{rb}$.

monosperma Willd. a $\mathrm{rb}$

horrida Willd. arb.

fpecies nova fpinis aureis arb.

Glob b a mutans Willd. W

Globularia cordifoliag. 


\section{$-28-$}

Globulari vulgaris . $_{\text {. }}$ nudicaulis $g$.

Gloriofa fuperbd w.

Gloxinia maculata L. Herit. w.

Glycine caribaea Jacq. w.

monoica w.

rubicunda Curt. g.

frutescens a rb.

Gnaphalium ericoides g.

Stoechas $g$.

orientale $\mathrm{g}$.

mucronatum $\mathrm{g}$.

cymofum $\mathrm{g}$.

helianthemifolium g.

Gnidia pinifolia $g$.

fimplex $g$.

Goodenia ovata $g$.

lanceolata $\mathrm{g}$.

Gorteria rigens $\mathrm{g}$.

laciniata $g$.

pectinata g.

Grew i a occidentalis $g$.

Guajacum afrum $\mathrm{g}$.

$\mathrm{Guil}$ andina dioica a $r$ b.

Haemanthus punicens $w$.

$$
\text { coccineus } w \text {. }
$$

Hi kea glabra $g$.

$$
\text { pubescens } g \text {. }
$$

Halesia tetraptera a rb.

$$
\text { diptera arb. }
$$

Halleria lucldag

Hamamelis virginiana arb.

II a mell ia chryfantha Ja c q. w.

Iledera Helix arb. 


\section{$-29-$}

Hedera Helix fol. variegatis arb.

quinquefolia ar b.

cordata arl.

Hedyfarum pictum w.

$$
\begin{aligned}
& \text { maculatum w. } \\
& \text { coronarium g. } \\
& \text { f albo } \\
& \text { vespertilionis w. } \\
& \text { heterocarpon w. }
\end{aligned}
$$

Helianthemum Lippi g.

Heliconia humilis Willd. W.

Heliocarpus americanus w.

Heliotropium peruvianum g.

Hemerocall is coerulea 17 illd. g. japonica Thunb, g.

Hemimeris coccinea $g$.

$$
\text { urticaefolia } y \text {. }
$$

Hermannia holosericea g.

$$
\begin{aligned}
& \text { lavandulifolia } g \text {. } \\
& \text { hyfsep folia } g \text {. } \\
& \text { althaeifolia } \mathrm{g} \text {. } \\
& \text { micans Schrad, g. } \\
& \text { ciliata } g \text {. } \\
& \text { derudata } g \text {. } \\
& \text { fcordifolia } g \text {. } \\
& \text { difticha } g \text {. } \\
& \text { praemorfa } g \text {. } \\
& \text { alnifolia } g \text {. } \\
& \text { flammer } W \text { illd. } g \text {. } \\
& \text { angularis } W \text { illd. } g \text {. }
\end{aligned}
$$

Hernandia Sonora w. Hibiscus fpeciofus, w. esculentus w.

Manihot $w$.

Rofa- finenfis w.

f. plenc 


\section{- 30}

Hibiscus f.carneo

$$
\begin{aligned}
& \text { flavo } \\
& \text { populneus g. } \\
& \text { fyriacus a } r \text { b. } \\
& \text { fl albo } \\
& \text { f. violaceo } \\
& \text { f. pleno rubro } \\
& \text { f. plano albo } \\
& \text { fol. variegatis } \\
& \text { paluftris f. } \\
& \text { virginicus f. }
\end{aligned}
$$

Hippia frutescens $g$.

Hippophae rhamnoides arb.

$$
\text { canadenfis arb. }
$$

Houstonia alba $g$.

Hydrangea arborescens a r b.

$$
\begin{aligned}
& \text { nivea } \mathrm{Mich} \text {. a } \mathrm{r} \text {. } \\
& \text { opulo:des } D u \text { h. a rb.g.etarb. } \\
& \text { flupe coerulieo } \text { ? }^{\circ}
\end{aligned}
$$

Hyoscyamus aureus $g$.

Hypericum Androfaemum a 1 b.

$$
\begin{aligned}
& \text { clatum Aiton, arb. } \\
& \text { prolificum arb. } \\
& \text { Ḱalmianum a r b. } \\
& \text { tomentofum } \mathrm{g} \text {. } \\
& \text { calycinum a } \mathrm{r} b \text {, } \\
& \text { Afiyron arb. } \\
& \text { olympicum arb. } \\
& \text { hircinum } a \mathrm{r} b \text {. } \\
& \text { monogynum } \xi^{\circ} \\
& \text { balearicum g. } \\
& \text { canarienfe g. }
\end{aligned}
$$

Iypoxis erecta g. 


\section{$-3 I$}

I atropha urens w.

$$
\text { gorsypifolia w. }
$$

I as min u m grandiflorum $\mathrm{g}$.

f. pleno $g$.

Azoricum g.

odoratiffimum $g$ :

gracile w.

officinale $a r b$.

fol. varieg. 8 ,

humile a rb.

fruticans ar b.

Ib er is faxatilis f. et $g$.

femperflorens $\mathrm{g}$.

lllecebrum ramofum $g$.

Ilex aquifolium arb.

echinatum g.

fol. aureo varieg. $g$.

fol. arg. varieg. g.

fol, aureo varieg. echinat. g.

heterophyllum g.

Dpaca A iton $g$,

Caffine $g$.

anguftifolia $\mathrm{g}$.

myrtifolia Walt. g.

canadenfis $M i c h$. arb.

maderienfis $\mathrm{Duh}$, arb.

vomitoria Ait. g.

laurifolia $g$.

aeftivalis $\mathrm{L} \exists \mathrm{m}, \mathrm{g}$.

balearica $\mathrm{g}$.

Perado Ait.

Indig ofera pforaloides g.

In ula glutinofa $g$. hirfuta w:

crithmifolia $\mathrm{g}$.

I resine celofioides $\mathrm{Lam}$ g. 


\section{$-32-$}

I $x$ is tuberofa $g$.

fuperba $g$.

fimbriata $\mathrm{g} \cdot$

* Itea virginica a $\mathrm{b}$.

luglans alba ovalis arb.

cordiformis arb.

glabra Willd. a r b.

cinerea $a r b$.

$$
\text { ovalis a } \mathrm{rb} \text {. }
$$

compreffa Willd. ar b.

nigra a r b.

oblonga a $\mathrm{r}$,

reģia arb.

$$
\text { maxima a } r b \text {. }
$$

anguftifolia $A$ it. arb.

Pecan Marsh. arb.

fraxinifolia $\mathrm{Lam}$, art.

Iuniperus communis ar $\mathrm{b}$.

fuesica Horto par, arb.

phoenicea a $\mathrm{r} b$.

oxycedrus arb.

bermudiana a $\mathrm{r}$.

virginiana a $r b$.

thurifera a $r$.

* chinenfis a rb.

tamariscifolia a $\mathrm{rb}$,

proftrata a $\mathbf{r}$.

Sabiua a r b.

fol. varieg. $a \mathrm{rb}$.

remota $W$ end l. arb.

rana $W i l l d$, a r b.

I uffieua repens $w$.

Iuftitia adhatoda $g$.

pulchra w.

coccinea w.

hyfsopifolia $\mathrm{g}$. 


\section{- $33-$}

I uft i ci a nafuta w.

bracteolata W.

furcata w.

caracaffana w.

biflora w.

quadrifida w.

Ixia feneftratag.

crocata $g$.

Ix ora alba w:

coccinea W.

Kaempheria Galanga is,

$\mathrm{K}$ alanchoe fpithulata w.

Kalmia latifolia a r b.

络

anguftifolia $a r b$.

ramis horizontalibus

glauca a $r b$.

$\mathrm{Kiggellaria}$ africana $\mathrm{g}$.

Kitaibelia vitifolia Willd. g.

Koehlreutera paniculata a rb.

Lachenalia tricolor g,

luteola $\mathrm{g}$.

quadricolor $\mathrm{g}$.

viridis $\mathrm{g}$.

ferotina $g$, et $f$.

* Lagerstroemia indica g. et arb,

Lantana trifolia w.

Camara w.

flore violaceo $\mathrm{W}$.

odorata W.

recta w.

nivea w.

involucrata iw:

E.avandula multifida

pinnata $\mathrm{g}$.

Roechas g. 
Lavandula dentata g. abrotanoides g.

Lavatera Olbia g.

$$
\begin{aligned}
& \text { micans g. } \\
& \text { maritima } g \text {. } \\
& \text { arborea } g \text {. }
\end{aligned}
$$

La а r $\mathrm{s}$ nobilis g.

fol, varieg. g.

Borbonia g.

Safsafras g.

indica $g$.

Benzoin arb.

Ledum paluftre arb.

latifolium $\mathrm{g}$.

thymifolium $g$.

Leprospermum Thea Willd.

flavescens $\mathrm{S}$ mith, $\mathrm{g}$.

pubescens Willd. g.

juniperinum $\mathrm{S}$ mith. $\mathrm{g}$.

ambiguum $S \mathrm{mith}$, g.

punctatum g.

canescens g.

acuminatum? $\mathrm{g}$.

rubicaule $g$.

L. iguf $r$ u m vulgare a $b$,

$$
\begin{aligned}
& \text { c. fol. vatieg, } \\
& \beta \text { italicum } \\
& \lambda \text { latifolium }
\end{aligned}
$$

Limodorum Tankervillias Ait, w. album w. purpureum v.

Limonia trifoliata $w$.

Liquidambas Styraciftum arb. orientale a rb. 


\section{- 35.}

* Liquidambar asplenifolium arb.

Liriodendron tulipifera arh.

Lifianthus latifolius $g$.

$$
\text { * integrifol, a } x \text {. }
$$

Lobelia longifora w.

bellidifolia $g$.

cardinalis $\mathrm{g}$.

fulgens $g$.

Siphilitica g. et $\mathrm{f}$.

Lonicera fempervirens a $r$ b.

Periclymenum a $\mathrm{rb}$.

« quercifolium

Caprifolium a $\mathrm{r}$.

$\beta$ fol. variegat.

dioica $A$ it. a r b.

grats Aito a r b.

rotundifolia $a r b$.

balearica a rb.

etrusca arb.

praecox a $r$.

in:plexa a $\mathrm{r} b$.

Xyiofteum a rb.

nigra a r b.

alpigena $a \mathrm{rb}$.

coerulea ar b.

tatarica a r b.

A. albo

pyrensica a $\mathbf{r}$.

Symphoricarpos at $b$.

Diervilla arb.

Lotus jacotaeus g.

creticus g.

hirfutus a r b.

rectus $a \mathrm{r} b$.

Lod $w$ igia macrocarpa $g$

c. 2 
Lychuis grandifora g.

Lycium Bcerhaviaefolium g. europaeum arb. barbarum a $\mathrm{r}$. afrum $\mathrm{g}$. chinenfe $\mathrm{D} u \mathrm{~h}, \mathrm{arb}$, Spec. nova $g$.

Magnolia grandiflora $g$.

$$
\begin{aligned}
& \text { acuminata a rb. } \\
& \text { glauca a r b. } \\
& \text { tripetala a r b. } \\
& \text { discolor Jard. de Mălm, g. }
\end{aligned}
$$

Mahernia diffufa Jacq. $g$.

$$
\begin{aligned}
& \text { verticillata } g \text {. } \\
& \text { incifa J a c } q . g \text {. }
\end{aligned}
$$

Malva angufifolia $g$.

$$
\begin{aligned}
& \text { capenfis } g . \\
& \text { virgata } g \text {. } \\
& \text { afperrima } g \text {. } \\
& \text { miniata } g . \\
& \text { triloba } g \text {. } \\
& \text { leprofa } g .
\end{aligned}
$$

$M$ a urantia femperforens $g$ :

Medicago arborea $g$.

$$
\text { antirrhiniflora } \mathrm{g} \text {. }
$$

Melirfa fruticofa $g$.

Melaleuca fricta $g$.

hypericifolia Ven t. g:

ericoides $\mathrm{g}$.

fyphelioides Willd. g.

alba g.

myrthifolia $\mathrm{g}$.

Jucida L. Suppl. g.

thymifolia $\mathrm{S}$ mith. g,

Melastoma cymora w. 


\section{$-37=$}

Melia azadirachta $g$.

Melianthus major g.

$$
\text { minor } \mathrm{g} \text {. }
$$

Menis permum canadenfo a rb.

$$
\begin{aligned}
& \text { virginicum } a+b \text {. } \\
& \text { carolinianum } a r b \text {. }
\end{aligned}
$$

Mefembriantheinum elule $g$.

fpeciofum $g$.

fufcum $\mathrm{g}$.

ferratifolium g.

margaritaceum $g$.

imbricatum $g$.

glomeratum g.

corniculatum $g$.

aduncum $g$.

auftrale g.

carneum $g$.

compreffum g.

Cornuti g.

echinatum $\mathrm{g}$.

aequilateraleg.

infititium $\mathrm{g}$.

lunatum $g$.

maximum $\mathrm{g}$.

carnofum $g$.

uncinatum $g$.

glsucum $g$.

linguseforme $g$.

hispidum $\mathrm{g}$.

deltoides $\mathrm{g}$.

bellidiflorum $g$.

pinnatifium g.

falcatum $g$.

barbatum majus $\mathrm{g}$.

minus $g$. 
Mofembrianthemum cordifolium $g$.

fplendens $g$.

latum $\mathrm{g}$.

longum $g$.

compactum $g$.

caninum $g$.

albidum $\mathrm{g}$.

fiffum $\mathrm{g}$.

dolabriforme 8 .

loreum $g$.

clavatum $g$.

umbollatum $g$.

villofum $g$.

deflexum $g$.

craffiólium pallens

aurantiacum $g$,

maximum $g$.

lunatum $g$.

coccineum $g$.

pulcerulentum $g$.

folinfum $g$.

mutabile g.

pulchellum $g$ 。

roftratum $g$.

felinum $\mathrm{g}$.

calamiforme $\mathrm{g}$.

expanfum $g$.

tortuofum $\mathrm{g}$.

geniculiflorum g.

noctiflorum $g$.

bicolorum $\mathrm{g}$.

tuberofum $\mathrm{g}$. tenuifolium $g$.

ftipulaceum $\mathrm{g}$. verruculatum $g$. echinaิtum $g$. 
$M$ Gembrianthemum viridiforum $g$.

Spinofum $g$.

craffifolium $\mathrm{g}$.

glaucoides $\mathrm{g}$.

fpectabile g.

omarginatum g.

fcabrum $g$,

aureum $\mathrm{g}$.

ferratum g.

bracteatumg,

pallens g.

pugionifo:me g.

filamentofum $\mathrm{g}$.

acinaciforme $g$.

forficatum $g$.

parviforum $g$.

Mespilus Amelanchiér ar be

canadenfis arb.

Chamaemespilus a $\mathrm{b}$.

arbutifolia a r b.

Cotoneafter a $\mathbf{r}$ b.

Pyracantha arb.

japonica g.

germanica erecta ar $b$.

ce diffura arb.

$\beta$ fruct, maximo arb.

Keffarfchmidia fruticosa $g$. Metrosideros citrina Curtis g.

linearis $\mathrm{Smith} \mathrm{g}$. pinifolia $\mathrm{g}$.

glandulofa $g$.

lophanta Vent. g.

latifolia $\mathrm{g}$.

diosmaefolia $\mathrm{g}$. 
Metrosideros lanceolata Smith, g.

Mimofa arborea g.

$$
\text { nodofa } \mathrm{g} \text {. }
$$

Catechu g.

longifolia $g$.

obliqua $\mathrm{g}$.

verticillata $g$.

floribunda $g$.

latifiliqua wa

virgata $w$.

glauca w. .

Lebeck w.

farnefiana $\mathrm{g}$.

tamarindifolia $w_{0}$

anguftifolia w。

pudica $w$.

fenfitiva w.

plena w.

pennata w.

julibricenfis $\mathrm{g}$.

Ceratonica w.

Mirabilis dichotoma $g$.

Mitchella repens arb.

Monfonia fpeciofa Willd, $g$,

Moraea chinenlis $f_{0}$ et $g$ *

iridisides $\mathrm{g}$.

northiana $\mathrm{W}$.

Morina perfica $f$, et $g$.

Morind a Rayoc, Jacq. w.

Morus alba arb.

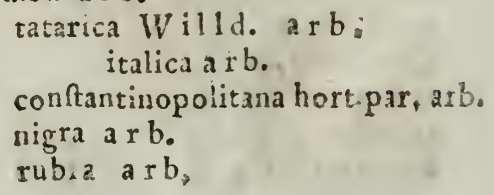




\section{- AI}

Morus papyrifera ot et 우 arb.

Mu fa paradifiaca w. coccinea Andrews, wo

MIyrica quercifolia $\mathrm{g}$.

cordifolia $\mathrm{g}$.

Faya Ait. g.

fegregata $\mathrm{g}$.

banchliaefolia g.

trifoliata $g$.

cerifera a $r$,

Gale ar b.

Myrfine africana

laciniata g.

retufa g.

Myrtus macrophylla $g$.

$$
\begin{aligned}
& \text { flore pleno } g \cdot \\
& \text { acuminata } g \cdot \\
& \text { humilis } g . \\
& \text { microphylla g. } \\
& \text { brevifolia } g \text {. } \\
& \text { rotundifolia g. } \\
& \text { acuminata g. } \\
& \text { fol. variegatis } g \text {. }
\end{aligned}
$$

Nerium Oleander $g$

$$
\begin{aligned}
& \text { flore albo g. } \\
& \text { flore carnou g. } \\
& \text { indicum w. } \\
& \text { tlore pleno w. } \\
& \text { odorum w. }
\end{aligned}
$$

Nyctanhes Sambacw.

$$
\text { A. pleno } W \text {. }
$$

Ny fra villofa Mich, $a^{2} \mathrm{r} b$;

candicans Mich.w.

aquatica arb.

glauca a $b$, 
$O$ cym um gratiffimum w,

$\mathrm{Deder}$ a prolifera $\mathrm{g}$.

Olea europoea $x$.

rotuudifolia $g$.

fragrass $y$. caperfis.g. undulata $\mathrm{g}$.

Ononis fruticofa arb.

Origanum D.ctamnus $g$.

aezyptracum g.

Ornithogalum narbonenfe $g$.

caudatum \&

Oft o os permum coeruleum $g$. moniliterum $\mathrm{g}$.

$\mathbf{O}$ thonna cheiranthifoliag.

Oxalis incarnata $y$.

Pancratium maritimum

$P_{a}$ fferina lateriflora $g$. iil

P a friflora coerulea $g$.

laxa g.

erecta $\mathrm{g}$.

lutea $f$.

longifolia w. capfular's $w$,

fuberofa w.

glabra w.

hirta w.

normalis $\mathrm{W}$.

biflora w.

alata $\mathrm{W}$.

punctata $\mathrm{W}$,

ferracifolia W.

perfoliata $w$.

respertilionis $\pi$.

glauca $1 \%$.

incarnata v?. 
Paffiflora adiantuin w.

Pavonia fpinifex Willd, w.

priemorfa $g$.

Pentzia flubellitormis $\mathrm{g}$.

Periploca graeca arb.

Petiveria alliacea w.

$\mathrm{Phil}$ adelphus inodorus arb.

$$
\begin{aligned}
& \text { laxus arb, } \\
& \text { coronarius arb. } \\
& \text { fore pleno w. } \\
& \text { fol. variegatis } w_{0} \\
& \text { humilis w. }
\end{aligned}
$$

Phillyrea mediag.

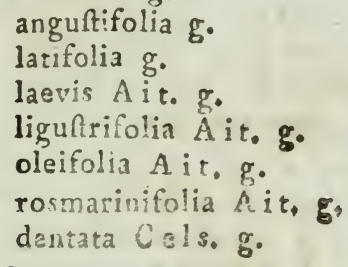

Phlomis Leonurus o. * fruticora arb. 2 purpurea a r b.

Phlox fubulata 8 . fuffruticosa $\mathrm{g}$. fibirica $\mathrm{g}$. fetacea g. fuzveolens g. ftolonifera $\mathrm{f}$, et $\mathrm{g}$.

Phoenix Dactylifera w.

Phormium tenax

Phylica ericoides g. capitata g. ftipularis $\mathrm{g}$. callofa $g$. 
Phylica pubescens $g$.

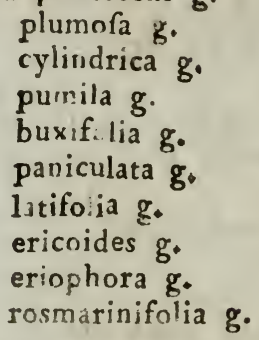

Phyllanthus grandifolius w.

Phyll is Noblag. ipeciofus $w$.

Phyfalis flexuofag.

$$
\begin{aligned}
& \text { peruviana } g \text {. } \\
& \text { fomnifera } g \text {. }
\end{aligned}
$$

Phytolacca abyfinica w.

$P i a u s$ fylveftris arb.

$$
\begin{aligned}
& \alpha \text { rubra w. } \\
& \beta \text { montana w. } \\
& \lambda \text { tatarica w. }
\end{aligned}
$$

Pinea $g$.

Pineafter $\Lambda$ iton. arb. maritima du Roi a rb,

Toeda w.

rigida du Roi w.

Cembra w.

Strobus w.

echinata Mill, W。

\%

halepenfis $\mathrm{w}$.

palutris $\mathrm{Ai}$ t. W.

incps w.

laxa Ehrh. w.

Larix w.

nigra w. 
Panus Cedrus w.

$$
\begin{aligned}
& \text { Abics w. } \\
& \text { picea w. } \\
& \text { baifamea w. } \\
& \text { canadenfis w. } \\
& \text { mariana alba w. } \\
& \text { rubra w, } \\
& \text { orientalis w. }
\end{aligned}
$$

Piper reticulatum $w$. verticillatum w. obtufffolium w. blandum $w$. magnoliaefolium W, cuneifolium w. aduncum w. clufiaefolium w, triphyllum w. pereskiacf lium $\mathbf{W}$. med um w.

$P$ is cidia Erithrina w, Pistacia Lentiscus $\mathrm{g}$. Terebinthus $g$.

Pitcarnia anguftifolia $w$. latifolia w.

Platanus occidentalis arb. orientalis w. a acerifolia w, $\beta$ hispanica w. $\lambda$, quercifolia

Plectranthus fruticofus L. Her, g. Plumbago rofea w. Zeylanica? w

Pollichia campeftris g. Polygala Chamaebuxus $f_{0}$ et $g$. 
Polygonum frutescens $a x b$.

Polymnia Wedelia g.

Polypodium aureum w.

Pontederia cordata g.

phymatodes w.

Populus alba arb.

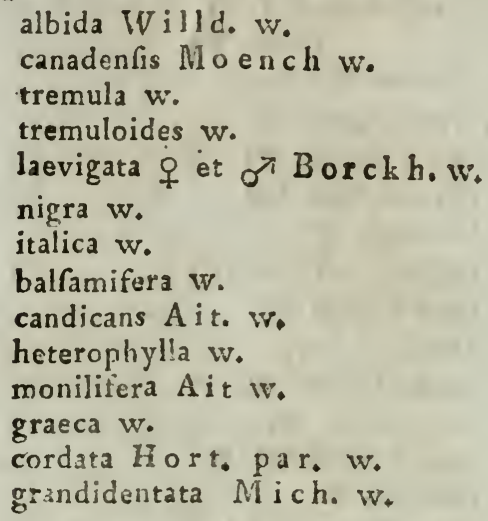

Portlandia hexandra w.

Potentilla fruticufa arb.

Poterium fpinofum $\mathrm{g}$.

Pothos craffinervia w.

Prafium majus g.

Prinos verticillatus $a r b$.

* glaber arb.

laevigatus I.odd, a rb.

Protea pallens $g$.

faligna $\mathrm{g}$.

divaricata $\mathrm{g}$.

conifera $g$.

linearis $\mathrm{g}$.

hirta $g$.

cinerea $g$. 
Protea tomentofa $g$.

torta $\mathrm{g}$.

siliata g.

carnofa $g$.

caudits $\mathrm{g}$.

Prunus armeniaca arb.

da.ycarpa Ehr, if.

ipiciofa W.

infititia W.

domeftica $\mathrm{IV}$.

oeconomica B orckh. Tr.

fyriaca Bor ckh. W.

italca Horckh. IV.

cerafiform.s Ehrh. IV.

Injrobalina $H$. pir. W.

finenfis $H$ par. WV

perficifolia camadenfis $\mathrm{W}$.

candicans IV.

puriala w.

prufrata IV.

domeftica obortiva IV.

fire pleno w.

pendula Wend 1 . W.

Cerafus W.

fore pleno W.

fol. variog. W.

Semperflorens Ehrh. W.

fruticofa Pallas IV.

Avium w.

Padus TV.

$$
\text { flore plano w. }
$$

¿ rubra IV.

$\beta$ nigra $\mathrm{k}$.

$\gamma$ fol. variegatis $W$.

virginisna $\mathrm{W}$.

sezotina Willd. Wo 
Prunus Mahaleb. w.

fructu flavo $w$.

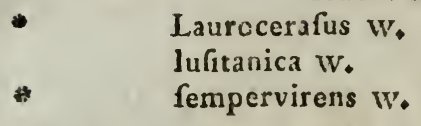

Pfiadia glutinofa Willd, g.

Pfidium pyriferum w.

Pforalea bituminofa $g$.

pentaphylla $g$.

tenuifolia $g$.

pinnata $g$.

hirta g.

Pteris cretica w.

ferrulata w.

Ptelea trifoliata a $r b$.

Punica granatum $\mathrm{g}$.

flore pleno $g$.

flore albo pleno $g$.

flore flavo g.

Pyrus Malus fylveftris a rb. praecos Pallas W. domeftica $\mathrm{W}$. apetata Münch h, W. fpectabilis w.

baccata $\mathrm{W}$. f. pleno w. coronaria W. fructu nigro $w$. fol. aureo varieg, W. hybrida Hort. Par. W,

$*$ fempervirens $w_{*}$ upsala W.

Communis a $\mathbf{r b}$,

Pyrafter w. flore pleno W\% 


\section{$-49-$}

Pysus Malus falicifolia H. par, ar

Montis Sinai id.

Michauxia H. par. id.

pollveria H. par. id.

Cydonia maliformis id.

pyriformis $d$.

Jufitanica $H$. par. id.

finenfis H. par, id.

Quaffia excelfa id.

Quercus Roburid.

a fefile arb.

$\beta$ fol. varieg. id.

faftigiara Hort. par, id.

Cerris id.

$\alpha$ frondofa Ait. id.

$\beta$ bullata Ait. id.

$\gamma$ finuata $A$ it id.

$\delta$ crispa id.

discolor du Roi id.

Prinos $\alpha$, tomentofa Mich. a r b.

monticola Mich, arb.

paluftris Mich. ar b.

acuminata Mich. id.

Prinoides Willd, id,

ร

heterophylla Ait. id.

Phellos anguftifolia id.

latifolia id.

Ilex $\mathrm{g}$.

Suber g.

coccifera a $b$.

gramantia Willd. arb.

virens a maritima Mich. id.

nigra a r b

pubescens id.

rubra id. 
Quercus rubra $x$ minor arb.

Alba id.

Fsculus id.

Aegilops id.

tinctoria in ich id.

obtufiloba Nlich. id.

macrocarpa Mich. id.

Banifteri Mich. id.

coccinea $W$ ang. id.

aquatica $A$ i to id.

paludofa iv.

haliphloecs H or'. par. id.

velutina id.

montana Willd. id.

maritima $W i l l d$. id.

triloba Mich. id.

Caftanea Willd. id.

repanda Willd. id.

Rhamnus catharircus a rb.

infectorius id.

faxatilis id.

$*$

fempervirens Hort. par. id,

lycioides $\mathrm{g}$.

ignaneus $\mathrm{g}$.

hybridus L. Horit. arb.

buxifolius $g$.

* burgundica arb,

Alaternu's arb.

fol. aureo varieg. $g$. fol. arg. varieg. $g$.

fol. maculatis g.

laciniata $\mathrm{g}$.

Dalearica D uh. arb. $g$.

Frangula a r b.

tranculoidos $\mathrm{M} \mathrm{i} \mathrm{ch.} \mathrm{w.}$

lDinus w.

Poumilus w. 
* Rhamnus Paliurus arb.

* Ziziphusid.

finenfis $L$ a m. $g$ 。

* lineatus arb.

Rhodor a canadenfis a $\mathrm{b}$.

$R$ hapis flabelliformis w. acaulis $\mathrm{W}$.

* Rhododendron maximum a a b. : fl, albo id.

* ponticum id.

* purpureum id. punctatum Willd, g. ferrugineum $a r b$. hirfutum id.

Rhus viminalis g. oxyacanthoides Ju $\int s_{*} g$. lucidum $g$. quercifolitem g. Coriaria arb. glabrum w. typhinum w. Copallinum IV. elegans $A i t . ~ W o$

Vernix w.

Metropium g. radicans a $\mathrm{r}$ b. Toxicodendron id. Cotinus id. pumilum Mich, id.

Ribes rubrum arb.

fructu maximo rubro id. albo id, carneo $\mathrm{V}$. petraeum Willd. arb. tpinum $2 \times b$. 


\section{$-5^{2}$}

Ribes nigrum arb.

$$
\text { fol. varieg. id. }
$$

glandulofum $P$ er roon id.

diacantha $W$ illd, id.

reclinatum id.

Groffularia id.

Uva Crispa id.

Oxyacanthoides id.

Cyuosbati id.

proftratum L, Herit. id.

floridum id.

penfylvanicum $W$ ill d. id.

patagonium. S p. nova id.

floridanum $W$ illd. id.

Rivina laevis w.

humilis $w$.

brafilienfis w.

Robinia Pfeudoacacia arb.

$$
\text { fl. violaceo id. }
$$

inermis id.

viscofa $D$ uh. a r b. id.

inermis $H o r t . P a r, i d 。$

hispida ${ }_{1} d$.

Caragana id.

pygriae, id.

frutescens id.

fpinofa id.

Chamlogu Lam. id.

Halodendron d

$\beta$ triflora L. Herit. id. altıgana $L$. Herit. id.

Rondeletia americana d.

$R \circ f a$ alba $L$ arb

$$
\begin{aligned}
& \alpha \text { femiplena Roefsig id, } \\
& \beta \text { plena id, 1d, }
\end{aligned}
$$


R ofa alba $\gamma$ incarnata id. a $r b$. amabilis id. id. alpina $L$. id.

$\alpha$ lagenaria id.

$\beta$ fo. pleno albo id.

$\gamma$ fl. pleno rubro id.

bracteata $g$.

beigica du Roi a 1 b.

incarnata id.

rubicans id.

burgundica $R$ oefs id.

$\alpha$ major id.

$\beta$ humilis id.

$\gamma$ Kermefina id.

Centifolia L. id.

flore fimplici id.

unica $R$ oefs id.

apiifolia id, id.

carnea id.

decora id.

muscofa Aiton. id.

anglica $\mathrm{W}$.

rubicans Roefs id.

prolifera id. id.

major id. id.

minor $\mathrm{K}$ o e $f s$ id.

corymbofa id.

crenata id.

ranunculoides id.

rufescescens id.

corymbofa Ehrh. jd. carolina du Koi id.

cinnamomea $\mathrm{L}$. id.

f. pleno Roefs id. 


\section{$-34-$}

* Ro ra chinenfis Willd. id,

flore albo $\mathrm{g}$.

canina L. $a \times b$.

f. pleno Roefs. id.

carnea id. id.

damascena rubra $\mathrm{A}$ i t o $\mathrm{n}$ id,

alba $R$ oefs. id.

dolofa $W$ endl. id.

diacantha Pall as id.

elongata $\mathrm{W}$ end $\mathrm{l}$. id.

Egianteria pumila L. id.

plena id.

major id.

francofurtenfis Münchh。id。

fraxinifolia id.

glaucophyila fo. pleno id.

gallica $L$. id.

humilis Wendl. id,

indica $g$.

holosericea fimplex du Roi arb.

maxima Roefs id.

regalis id.

multiplex id.

Kamtschatica Vent. id,

lucid $\mathrm{E} h \mathrm{rh}$, id.

lactea fl. pleno id.

lutea L. id.

plena minor Roefs, id.

major id.

multiplex Du Koi id.

auftriaca $R$ oe fs. id.

senfrua $W$ illd. id.

corymbofa Roefs id.

alba id. id.

variegata id.

Porzlandica id. 


\section{$-\quad 35$}

Rofa melitevris $R$ oe $\{s$ arb.

* mofchata femiplena id.

* plena id.

pimpinellifolia rubra $L$. id.

albs id!.

fpinofiffiuna li:it.

f. pleno is!.

praecox $W$ end $l$ id.

provincialis rubicans $R$ oefs irl.

regalis id.

rubra ic.

prolifera id.

marmorea id.

pallida ic.

hollandica id.

rubiginofa L. id.

duplex $K$ of $s$, id.

regina maxima id.

minor id.

femfervirens $r$

fefsilifiors arb.

flore pleno id.

* femperforens Willd. id.

fcotica id.

ferpers Ehrh, id.

fcandens Mioenh, id.

villore $\mathrm{L}$. id.

flore piena id.

Vilmorin id.

virginiana plena $R$ oefs id.

Rosmarinus officinalis $g$.

anguntifolia $\mathrm{g}$.

Royena hirfuta $\mathrm{g}$.

laevis $\mathrm{g}$. 
Rubus odoratus arb.

idjeus id.

fructu albo id.

fruct: maximo id.

fructu max, côrneo id.

femperfurens id.

f. rubro pleno id.

fruticifus id.

jacioiarus id,

fl. pleno id.

laevigatus id.

tomentofus $W$ e $t$. flora id.

occidentalis $\mathrm{Mich}$ id.

villofus $\mathrm{Ait}$, id,

Caefius id. inermis id. vulpinus id.

faxatilis id.

arcticus id.

Chamaemorus id. ruscifolius $g$.

Ruelli a villofá w. varians $\mathrm{W}$.

glaudestina $g$.

R umex Lunaria g.

* Kuscus aculeatus arb.

* Hypophylum id.

* Hypogloffum id.

* racemofus id.

Ruta montana arb.

graveolens id.

$S$ a ccharum efficinarum id.

Salicornia fruticofa $g$.

Salix triandra arb.

pentandra id. 


\section{$-57$}

Salix pentandra fol, varieg, arb.

vitellina id.

amygdalina id.

hattata id.

fragilis id.

babylonica id.

purpurea id.

Helix id.

myrfinites id.

a fericea id,

arbus cu 3 id.

retufa id.

reticulata id.

incubacea id.

aurita id.

fol. varieg. id.

lanata id.

lapponum id.

arenaria id.

depreffa Hoffo id,

repens id.

fusca id.

rosmarinifolia id.

Caprea id.

fol. varię̧ id

viminalis id.

cinerea id.

alba id.

decipiens Hoffm, id.

acuminata id.

rubra Willd. id.

helvetica d.

philicifolia Hoffm. id,

daphnoides Villars id,

caefia Villars id.

Gilefiaca Smidt, id, 
Salix coucolor arb.

glaucescens id.

nigricans id.

Halleri id.

venulosa id.

americana $W$ end 1 . id.

bicolor $\mathrm{E} h \mathbf{r h}$. id,

pruinosa Wendl. id.

ferotina id.

undulataid.

fina? id.

mollifima? id.

ambigua ? id.

hermaphrodita? id.

$S$ als ol a fruticosa $\mathrm{g}$.

Salvia formora $g$.

coccinea $w$.

canarienfis $\mathrm{g}$.

cretica $g$.

repens $\mathrm{g}$.

aurea $g$.

paniculata $g$.

pinnata $g$.

mexicana $g$.

officinalis a r b.

Sambucus nigra aṛb.

$$
\begin{aligned}
& \text { fol, varieg. id. } \\
& \text { fol.aur, var.id, } \\
& \text { fructu albo id, } \\
& \text { viridi id. } \\
& \text { laciniata id. }
\end{aligned}
$$

canadenfis id.

racemosa id,

* Santolina Chamaecypariflus arb. rosmarinifolia $\mathrm{g}$. 


\section{$-59-$}

Santolina anthemoides $g$.

$S$ a pind us Saponariag.

Satureja Thymbra g:

Samol us Valerandi g.

Saxiiraga farmentosa $g$.

fragilis $\mathrm{g}$.

multiflora g.

Schinus dependeris $g$.

$S c a b i o f a$ cretica $g$.

africana $\mathrm{g}$.

Scilla peruviana $\mathrm{g}$.

$S$ edum Scheuchzeri $g$.

populifolium arb.

Selago corymbofa $g$.

Sempervivum glutinofum

arboreum g.
fol. varieg. $g$.

arachnoideum $g$. et $f_{\text {, }}$

Senecio elegans fl. pleno $g$.

longifolius $\mathrm{g}$.

halimifolius $\mathrm{g}$.

Sida indica w.

molliffima $w$.

Sideritis canarienfis $g$.

Sideroxylon lycioides g.

$$
\text { tellax } g \text {. }
$$

* Silene fruticofa arb.

Sisymbrium millefohum $\mathrm{g}$.

Sifyrinchium palmifolium

Bermudiana g.

convolutum $\mathrm{g}$.

$S \mathrm{midia}$ fruticofa $\mathrm{g}$.

Smilax aspera arb.

pseudo China g.

caduca $\mathrm{g}$. 
Smilax excelfag.

Solanum ver'ascitolium $g$.

Pfesdocapficum g.

pyracanthoides $g$.

macrocarpo: W.

diphyllum w.

bonariense $\mathrm{g}$.

marginat im go

vespert lionis $g$.

Sodomeum $\mathrm{g}$.

tomentofum $g$.

campechienfe w.

Soldanella alpina $g$. et $f$.

Sonchus frutcoius $g$.

arbcreus $g$.

Sophora microphylla $g$.

tetraptera $\mathrm{g}$.

japonica arb.

Sorbus aucuparia id.

domertica id.

hybrida id.

$S$ parrmannia africana $w$.

Spartium junceum arb.

plenum $g$.

monospermum $g$.

fpinofum $g$.

lufitanicum $\mathrm{g}$.

radiatum $a \mathbf{r} b$.

furgans $g$.

fcoparium a $\mathbf{b}$.

$S p h a e r a n t h u s$ indicus $g$.

Spigelia marylandica $g$.

$S p$ ielmannia africana w.

$S$ piraea falicifolia arb.

alba $a \mathbf{r}$. 


\section{- OI -}

Spirraea alpeftris Borckh. arb,

Chamaedrito ia dd.

betulifoliz id.

opulifolia id.

laev.gita id.

hypericifolia id.

tenuifolia $d$,

crenata $1 d$.

triloba id.

forbifolia id.

tomentofa id.

lohita $f$ et $g$.

trifoliata $f$, et $g$.

thalictro:des arb.

ulmifolia Willd. id.

corymbofa $W$ endl. id.

i? flexa id

carpinifolia id.

oblongifolia id.

Stachys circinata $g$.

Staehelina Charaepeuco g.

-Stapolia elegans $w$.

variegata $w$.

gemi.atr $w$.

grandiflora $w_{0}$

revoluta $w$.

Serora $w$.

campanulata $2 \%$.

cespitofa $w$.

hirluta $w$.

asterias $w$.

irrorata $w$.

pallida $w_{0}$

ciliata $w$.

veluta 
Stapelia mixta $w$.

verrucosa w.

reclinata w.

reticulata $w$.

orbicularis w.

rugofa w.

fellata $w$.

Staphylea pinnata arb. trifoliata $w$.

Statice fruticola $g$. monopetala g; mucronala $g$. tatarica $f$. latifolia $f_{0}$ et $g$. cephalotes $g$. globularifolia $\mathrm{g}$. oleifoliz g. trigona Palea f.

Sterculia platanifolia $g$. Stevia ferrata $g$. eupatorioides $\mathrm{g}$.

* Stew artia Malacodendron arb. pentagyna $g$.

Strelizia Reginae w. Struthiola erecta $g$.

$$
\text { virgata } g \text {. }
$$

Styrax officinale ar $\dot{b}$.

lasvigatum $\Lambda$ it. $g$. grandifolium Ait, $g$.

$S$ werti a perennis $g$. Syringa vulgaris ar $b$.

$$
\begin{aligned}
& \text { A. aibo id. } \\
& \text { f rubro id. } \\
& \text { fol. varieg. id. } \\
& \text { perfica w. }
\end{aligned}
$$




\section{$-0_{3}-$}

syringa thore albo arb.

$$
\text { laciniata id. }
$$
froenfis Willd. id.

Tabernaemontana Anfonia $g$.

'l a getes lucida g.

Tamarix germanica arb.

\section{gallica id.}

Tarchonanthus camphoratus g.

Taxus bacrata a rb.

$$
\text { fol. varieg. id. }
$$

elongata $g$.

Tetragonia fruticora $g$.

Teucrium Vî̆um g.

mafulietre $\mathrm{g}$.

fruticans $g$.

inultiflorum $g$.

lucidum $\mathrm{g}$.

betonicaefolium $\mathrm{g}$.

abutiloides $g$,

- flavum arb.

Thuja aphylla g.

orientalis a $\mathrm{r} b$.

$$
\text { a plicsta id. }
$$

occidentalis id.

$\alpha$ odorata id.

articulatd $\mathrm{D}$ \& h. arb. g。

Thymus maftichina $g$.

Tiarella cordifolia $g$.

Tilia microuhyila $D$ uh, arb.

a corallina id.

americana $W$ illd. id.

pubescens A iton. id.

alba Aiton。 id.

Tillandsia lingulatz 


\section{- $6 t-$}

Tournefortia foetidiffrma $w$.

h irfutiffima $w$.

volubils $w$.

Trachelium coeruleuin $g$.

Tradescantia discolor $q v$.

rofea w.

Tragopogon Dalechampii $g$.

Tropeol um hybridun: $g$.

Turnera ulmifolia $w$.

Tufsilago fragrans $g$.

Ulex europaeus arb.

Ulmus campeftris arb.

$\alpha$ fol. crispis id.

$\beta$ fol, maculatis id.

fuberofa $W$ illd.id.

fativa du Roi id.

crenata Hort $\mathrm{Par}$. id.

pumila $\mathrm{g}$.

effufa $R$ oth arb.

americana id.

pendula latifolia id.

Urena lobata $w$.

Urtica nivea g.

arborea $g$.

Uvularia amplexifolia g.

Vaccinium macrocarpor. $g$.

arbutiolium g.

penfylvanicum $g$.

punctatum $g$.

arboreum $\mathrm{g}$.

glaucum $g$.

amoenum $g$.

myrtillus a $r$.

Vitis-idaea id.

-xycoccos id. 
Verbascum Myconi g.

Verbena triphylla g. jamaiceufis $\mathrm{g}$. bonarienfis $g$. fricta $\mathrm{g}$.

Verbefina ferratifolia w,

Veronica decuffata $g$. frusiculofa arb.

Viburnum Lantana arb. ovata id.

综 acerifolium id. Caffinoides id. dentatum id.

$\beta$ humile id.

Ientagn $i d$. grandiflorum id. nudum id. prunifolium id. pyrifolium id. Opulus id fol. varieg. id. canadenfis id. rofeum id.

Tinus $\mathrm{g}$.

fol. varieg. g. latifolium $\mathrm{g}$. laevigatum $g_{0}$

yinca rofea w.

fl, albo w. minor a $r$ b.

A. violaceo id.

fl, albo id.

fol. varieg. id. major id.

Visgilia lutea $\mathrm{g}$. 
Viola hifora $f$.

$V$ ite $x$ Negundo $g$.

Agnus $C_{a}$ ftus a $b$.

tiffuli $\mathrm{tag}^{\mathrm{g}}$.

Vitis Vinifera a r b.

laciniofa id.

vulpina id.

Labrusca id.

arhorea id.

cordita id.

riparia Mich, id.

Volkameria aculesta w. inermis $\mathrm{w}$.

Wachendorfia thyrfiflora $g$.

W alt heria americ na w.

Wefiring i a rofmarinifolia $g$.

Wulfenia carinthiaca $f$. er $g$.

Xeranthemum bracteatum $g$.

retortum g.

Xyloply!l a fa'cata w.

arbuscula w.

pinnatifida $w$.

Y u c ca filamentofa a r b.

gloriofig.

aloeifolia

pentula w.

Draconis w.

$\mathrm{Zami}$ a pumila w.

Zanthoxylum trifoliatum $g$.

Clava Herculis ar b.

Zanthorhiza apiifolia L. Herit, arb.

Zygophyllum Fabago f.

tostidum g.

feffit folium $g$. 


\section{Schwetzinger 0 bft arte $n$ Vomfrühjahr 1809.}

\begin{tabular}{|c|c|c|}
\hline \multirow{3}{*}{$\begin{array}{c}\text { Aepf el. } \\
\text { Agatie-Aepfel rouge } \\
\text { blanc Sote, Enk- } \\
\text { huises agtie }\end{array}$} & \\
\hline & Chrift & Feb. Mirz \\
\hline & & \\
\hline Ananas & $\mathrm{Ch}$ & \\
\hline $\begin{array}{c}\text { Api la } \\
\text { Le }\end{array}$ & Duh & \\
\hline & $\begin{array}{c}\text { id } \\
\text { Chrift }\end{array}$ & \\
\hline & & \\
\hline & $\begin{array}{l}\text { id } \\
\text { id }\end{array}$ & \\
\hline & & \\
\hline & T. O Ag & \\
\hline$g$ & & $\begin{array}{c}\text { Jan. Au } \\
\text { id. }\end{array}$ \\
\hline er bohemi & & Dec. \\
\hline & & \\
\hline & & \\
\hline & & \\
\hline & & \\
\hline $\begin{array}{l}\text { plus grand } \\
\text { Sommer }\end{array}$ & T. Obftg & Septe \\
\hline
\end{tabular}




\section{A e p fel}

Borsdö fet klpiner Sommer grüner Winter

Bosfelder

Calville aromatique

d'angleterro

blauche d'été

d'hiver

d'fleur

musqué

herbft anis

Weifser herbit

rouge de Normandie d'été

Turque

rouge d'hiver

d'automne

rayé

etoilée

poir panaché

weifser italienifcher

Winter

rother Spitz Calville

Cardinal rouge

Canelle

Carolin d'angletterre

Chemife de foié blanche de foie rouge

Court pendu blanc

gris

gris le gros, ou Pomme de Berlin

rouge

rovale

platt

Coufinotte rouge d'ét
AutorenfReifzeit idem

Diel December

Chrift d. 2 Jahre

Diel Oct. Dec,

Chrift

id

Duham.

Diel

May

Aug. Sept.

December

Auguft

id September

id

T. Obng

Diel

jeft. Oct. id

Sept. Oct.

Duham. Juli

Diel

Quint. M. Nov. Jan, Chrift Oct. Nov.

T. Obftg. Auguft

Diel

May

T. Obftg. Nov. Merz

- id Dec, Aug. id

Chrift

Diel

Chrift

id

T. Obftg. D c. Febr.

C.d. Baum

Diel

Dec, April

Dec. April

Salzmann

Chrift. Jan. Febr.

Diel December

Chrift Dec. May

Diel Sept. Oct. 
A eptel

Coufinotte raycé d'été Derrinisca aus Jaffy

Eckapfel rother

Faros le gros

Fenouillet blanc

gros ou Pomme d'Anis le gros

rouge au Bardin

jeaune

$$
\text { ragé }
$$

Fleiner

\section{Winter}

grofser Winter

Francatu

braunrother

Frauenapfel brauner

Frauenrothacher, Pomme Chataigne

\section{Gehlapfel}

Gerftenapfel

Gewürz pfel weifser Somer Glasapfel grofser deutfcher Grosbon.

\section{Grunztapfel}

Graeu,lieher k'ein

Grobfen

$$
\text { gros }
$$

Gülderling dunkel fchwarzer englifcher englifcher Gold geiber holländif́cher rother

fchwarzer

rpanifcher Hartig Forelle
AutorenlReifzeit Diel Sept, Oct. Hacquet

T, Obftg. Dec. Juny Chrift. Uec. A I idem Dec. Febr. Duhamel id Chrift Nov. May Duham. Nov. Febr. Diel Dec. Jan. T. Obst. Merz April id Sept, Oct. Diel Dec. Fubr. id id

Cat.deChac Januar Diel Dec. Febr. T. Obrtg. Sept. Dec.

Baum. Cat. Dec. Juni T. Obftg. Sept. Oct. id Jul. Nov. Diel September id Apr. Sept.

Chrift

id Dec, Juni C. de Basle Dec, Febr. id id

T. Obftg. Dec, April

Chritt

Diel

Februar

Chrift Dec. Jan. id Jan. Febr.

Diel

Chrift. Febr. Merz T. Obfg, Nov. Febr. 


\section{A e p fel,}

Hartig grïn

$$
\text { gedrukte }
$$

Herrenapfel

Himbeerapfel leberrother harlemer rorher

Incomparable des Antilles Iungf rirapfel grofser

$$
\text { kleiner }
$$

Kantapfel faurer

$$
\text { enplifcher }
$$

Karlsapfel

Karthäufer gelber Winter Königsapfel

fränkifcher

Kräuter 'pfel, holläudifcher Kronapfel

$$
\text { ro:her Winter }
$$

Kurzer wufer. Pommed'A-

Lederapfel

$$
\text { vent? }
$$

Mätapiel ächter fchwarz-,

\section{brauner}

brauner

weifser

Mäucherapfel

$$
\text { rother }
$$

Malvafierapfel

Ma!zacher

Marck ipfel, rother

Marguer:te

Marienapfel fchöner

Mela de Rosmarino rougc

Melnnenapfel

Merveille de Portland
Autoren!Re ifzeit T. Obftg, Oct. April \begin{tabular}{c|c} 
id. & idem \\
Knoop & Nov. Dec.
\end{tabular} T. Obfg. id. Diel id. Chrift Dec. May T. Obftg. September id. id.

Kronp Dec, Oct. Diel id. Dec, Febr. id.

id. Nov Febr. id. November Chrift. Jan. Febr. id. id.

Sundrau IJdaurend T. Obit. Febr. Apr. Chrift Jan. Febr. IT. Obitg. Nov. April id. id. Cant. Bafel Dec. Febr. id. id.

T. Obftg. Dec. Merz C. Bafel Nov, Jan. Diel Nov. Dec. Manger Diel Sept.Febr. \begin{tabular}{c|l} 
id. & Aug. Sept. \\
Chrift & Nov. Febr. \\
id. & Jan. Merz
\end{tabular} Baum. Cat.jFeb. Iuli 
A e p fel. Aepfel.
Milchapfel braunfchweiger
Mutterapfel

Nezre d'oré

Ordensupf:1

Рaзrihapfel

Parmain Lean's

royal

Scharlach

Gelteeifter Sommer

Winter Gold

Sommar

Passe Pcmme blanche d'automne

Paftorapfel rouge d'automne

Pater nolter

Peppin fin d'angleterre (englifcher Gold Peppin) frenche

Newtons ou Reinette de Neuyork

d'or oupomme d'or grofser holländifcher

Gewürz

rofen

marmorirter Sommer

Silber

\section{weifter}

Pfingftapfel

Prund oder Tellerapfel

Pigeon rouge

$$
\text { b.anc }
$$

d'hiver

Pigeonnet rouge

$$
\text { royale }
$$

A u toren|Reifzeit

Diel

iJem Verzd.3J.

Chrift Dec. April

Diel Dac. Merz

Chrift Nov.April id. sept. Oct. id. Nov. Apr.

Diel

id. September

Chrift Nav Jan.

Diel September

Chrif Sept. Oct. id Ocrober

Diel Febr. Juli

Chr.ft Dec. Febr.

id Dec, Juli

Manger Jan. April

Chrift Jan. May

Disham Dac. Merz

Knoop idem

Chrift Jan. May

Diel Jan. Sept.

id Sept. Oct.

id Dec. April

id Nov. Merz

T. Obng. Sept.Febr.

Chrift. Nov. Febr.

id Dec. Febr.

id bis Merz

id Dec. Mirz

id Nov. Merz

Diel Oct, Merz 


\section{Aepfel.}

Pigeonnet blanc

Pilgrim klein r geftreifter grofser rother

Politerapf: I rcther

Pomeranzen $\mathrm{p}$ fel

Pomme d'Adam

d'uftems ou Streifing d'hiver

d'Aftracan ou Tr.ns.

blanche uiffe

$$
\text { parinte }
$$

de Canada

d Aout

de la chino â fleur dnu-

ble Pyrus fpect?-

bilis

Cocombre

Caracter ou Caracter

Citron

$$
\text { Reinette }
$$

de Carmes. Dortchens-

$$
\text { apfel }
$$

Double rouge de $\mathrm{Pa}$ racis douce de Bollurller
de drapd'or, vrat drap-

higue

de Grävenftein

hative avant toutes

de haute bonté

de Jerusalem

imperia!e vcrte

nonpareille

d'Orange
Autoren/Reifzeir

Diel October

T. ObRg. Dec April idem idm

Del Oct. Febr. id Dec. Juny id Jahre

T. Obfg.jDec. May
Chrift

Auguif

Whrift

Diel

\section{Wildenow Auguft}

Cat, Baum

\section{ヘuguft}

Diel Dec. Fubr.

T. Obfg. Nov. Apr.

Diel Dec. Febr.

Chrif Febr. Merz.

Baum Cat. Nov, May

Duham

Duham Nov. Dec.
Chrift Jan. April

Oct. Nov. id

Duham

Juli

Henne Dec. Fehr.

Diel Dec, Mierz

Duham Jan. Merz

Chrift Dec. Jan 


\section{Aepfel. Autoren'Reifzeit}

Pomme pors, wihrer Birn. C de Char Bec. Febr. förmiger

perfique

prium

royai d'angleterre

de at. Louis

de Strasbourg

Satine, Atlasłpfel

de $5 t$ Jacques

d. Sedan

taffetas

Violette

Pomocyrus

Poftophe d'hiver

d'eté

Prefent royal

Princeffe Noble

Princeffenatel englifcher

Pyrus Baccata

Quinceapfel (Quittonapfel)

Rabau weifser Sommer

rother Sommer

Rambour aigre

blanc d'automne

d'hiver

d'hiver

franc

Zurchauer rother win.

ter hat $\int 2$ Elle

Red Choic im Unfang.

Reinatte d'Aizerna

d'Angleterre la grofse

blanche

\section{8}

idem T. Obitg. Dec. M:y Bauirs Cat. Ende Aug.

Diel

Chrift Oct. Dec. T. Qbit. Auguft Diel Ost. Dec. id Dec. Febr.

Dunam Dec. May Chrift I J. dauert Duham Febr. Merz C. de Char October Manger

C. de Char Nove Jan. T. Obftg. Dec. April Linnei Chrift Nov. Dec. Diel id Dec. April Baum Cat. October Diel Dec. Jan. id Dec. Apri! Duhamel Aug. Sept. Chrift Dec, Merz id? id Jan. April Duham Dec. Nierz Diel INov. April 
Aepfel.

Reinette de Breda

de Bretagrae

Carpentin

de Ciffel

de Clareval

de Citron

Calville

a Cote veritable

de Carmes

dorée ou Reinetta jeau-

ne tardive

de Damafon

franche

$$
\text { doré }
$$

Forel'en

fiés (geftrikte Herbft-

Reinette)

Grise de Champagne d'hiver de Mletz la . etite

d'automne anRein Marbté

Greone

geftreifte

$$
\begin{aligned}
& \text { violet e } \\
& \text { rothe Sommer }
\end{aligned}
$$

glatte geibe

Gewürz

grüue Band (R. verte

$$
\begin{gathered}
\text { gelve } \\
\text { Zucker } \\
\text { herbft }
\end{gathered}
$$

de Harlem
A utoren'Reifzeir

Chrift Dec. Jan. Duham Nov, Dec. Chrift Dec. April

T. Orftg. Dec. Nay Chrift December Diel Dec, April idem Nov. Jan. Krehs Cat Dec. Merz T. Obstg. Jan. May

Duham Dec. Marz Diel December Duh. I Jahr Diel

Chrift

May

T. Obftg. Oct. Jan.

Duham. Febr. May

Baum Cat. Nov. April

Chrift I Jahr

Baum Cat November id. Dec. April

T. Otftg. Nov. Iuni Diel Nov. May id Aug. Sept.

T. Obftg Dec. Oct. Diel Nov. Jan.

T. Obftg. Nov. April Diel

T. Obftg. I Jahr Diel id id 


\section{$-75-$}

Aepfel.

Reinette horhzeits hyeroglyphen

jaune d'été

d' talse

Königliche

Kron

de Luneville

Montbron

Musquée

Meisner Alant

Nonpareille

(Sorte sonapfel)

de Normandie

Nelguin

d'Orleins

p cotée

piquée

poire

de Portugal

Parmin rouge

de Paris

de Rochelle

rouge

rouffe

$$
\text { de Sickler }
$$

roth graue Kelch

rosmarin

rothgefickte Mark

Satran

forte graue

forguliet

de fuifre

Triomphante

Verte
A utoren'Reifzeit

Diel Dec, April

idem November id Sept. Nov.

id

id Dec. Merz

id November

id 2 Jahr dau.

Chrift Jan. Febr.

id Dec. May

D.el October

Chrift Dec, April

id I Jahr

id Feb. Merz

id Januar

id Jahr

id Febr,Merz

id Oct. Jan.

Diel

id

Chrift

id Dec. Jan.

Cat deChar Dec. April

T. Obftg. id

Chr ft Dec. Febr.

Diel December

id Nov. Dec.

id Oct. Dec.

Basm Cut. Oct. Morz

Chrift Jan. Febr.

Diel Dec. Juny

Chrif

id

Dec. Merz

id

Jan, Juli 
A epfel.

Reinette Verte la grofse

de Windfor, ou ruyal d'angleterre

ZweijahrdaurendeZuk-

Zwerg

Revalfcher Birnapfel

Rheinapfel füfser

faturer

krislicher

Roi tres noble

Kofenhäger

Roftocker

Rothfeder

Rofette marbrée

Rofenftre fing schaumburg. Rofenapfe! Bendeleber rother Sommer

böhınifcher Summer

Scarlet agreable

$$
\text { ker Rein. }
$$

\begin{tabular}{|c|c} 
T. Obftg. & 2 Jahre \\
Diel & Dec. Merz \\
Chrift & Auguft
\end{tabular}

T. Obftg. Dec. May

Baum Cat. Novo April

Cant. Bafel Nov. May

Chrift Oct. Dec. id 0 t. Nov.

T. Obntg. Nov. April

Diel

id

id

ić

T. Obftg Auguft

Diel

Chrift

Schaisnafe

Scheurerapfel

Schmeerspfel fränkifcher

St. Floreat

Sebaftian, grofser, Caillol, rofat

Silberling

Soete Holard (Zimmetapfel)

Yoppon

Kentapfel

Sonappel, faurer

Spiegelapfel . holländifcher

Srreifing , ou Sträumerling Striemapfsl

St.ttiser rother

Autoren/Reitzeit

Chrift Jan. July

Folr. July

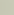




\section{A epfel.}

Stettiner weifser

grüıer

rother herbft

Süfsaplel gelber herbft rother herbit

Tafelapfel holländifcher rothgeftreifter

\section{weifser}

dunkelrother

Tafelapfel fürftlicher (Los-

Taubenfüschen kriger)

Tulpenapfel

Vecterapfel, ohne Kern Veilchenapfel Sommer

- herbft

Wachsspfel

Warafchapfel

Weilburger

Weinapfel

Wefterling

Wienerapfel

Winterapfel brauner

Würrapfel gelber

$$
\text { Nieifsner }
$$

Zehendheber

Zürcherapfel, gelberherbft $T$. Zuckerlimonien-Apfel

Zurchauer rother

Zwiebelapfel

Zwillingsapfel
A ut orentReifzeit T. Obftg. Oec. Merz Chrift Dec. Juny Diel Oct, Nov. idem Sept. Oct. id Sept. Jan.

Chrift Dec. Jan。

Diel Januar

T. Obftg. Nov, A pril

\begin{tabular}{|c|l} 
id & Auguît \\
Deel & October
\end{tabular}

T. Ohftg. Auguft id Oct, Dec. Diel December id Dec, July id id December Baum Cat. Sept. Oct. Diel Nov. Apr. T. Obfty. September id Nov, Apr. Diel Dec April Obftg. Sept. Oct, Chrift id Dec. Juny Diel Dec. Febr. 
Birnen.

Ah mon Dieu, cu Pomme

Ambrette d'hiver avec

fans épirés

Am're Joannet ou Poire da

Angel que de Bordeaux Apfeibirne

Auruitbirne deutfche

Belle et bonne

Bellufirie d'sutomne, ou

d'été. Supreme

d'hiver, ou Vermillon

Bergamotte d'Angleterre

d'auromne

de Bufly

Craffanne

panacheé
d'été, ou Miian d: la
beuvrere
d'Ho!lande ou Bær,
d'Alençon
de Paques
de Nice
ryeé ou Berg. fuiffe
Nunpareille
rougge
de Soulers
Sylsenge
quercy
runde Sommer
ler

Autoren! $R$ eifzeit Duham auf Sept. id id Nov Merz

Duham Ende Juni \begin{tabular}{c|l} 
id & Febr Merz \\
T. Obftg. & October \\
D el & Auguit \\
Chrift & Seprember
\end{tabular}

D.ham id

October Ju'y

'T. Obftg Nov. May Chrift Sept. Oct. Duham Oct Dec. Bzum Cat. Jan. Merz Un'am Nov. Dec. id

id id May Juny id Jan. Febr. Baum Cat.

Onh:m October

Chrift November id , Dec.

Duham Febr.Merz C de Char. Supt Oct. T. Obng. id A.gunt [Baum Cat.] October 
Birnen.

Beurré d'Angleterre da Bollwilier blanc, Doyene ou St.

d'oré Michel d'hiver de Metz Bзum Cat.

rouge

gris, le Beurré le petit d'Holiande romain

Bazy de Caiffoy ou Rousslet

\section{d'Anjou}

de Chaumontel ou Beurré d'hiver

\section{d'Hery}

de Montigny

de la Motte

Blanquet à longue queue le gros

rond

Blazbirne

Bonchretien d'Auch,Bonchr.

d'Espagne panaché

d'eté ou gratioli

d'hiver Musqueé

Bourdon musqué, Wespen-

Brunnenbirne birne

Calebafse Musqués

Caffolerte ou Muscat vert

Cattilac

Chair à Dame, Cher Adam
A u toren.'R e if́ze it Duham September Baum Cat. Apr. May Duham October Chrift Oct. Nov. Dec. Febr. T. Obftg. Ict Nov. Duham Sept. Oct. T. Obitg September \begin{tabular}{c|c} 
id & Sept. Oct. \\
Chrift &
\end{tabular}

Quintinye Nov.Febr.

Duham. id id Oct. Dec. id Sept. Oct. id Oct. Nov. id Augut id Baum Cat. id

Chrift Duham id Chrift Juli id October Duham id Baum Cat 1 October Manger Duham Diè Duham
Feb. Merz Nov. Dec. Auguft id Jan. May id Auguit Nov. Juny Augut 


\section{$=80$}

Birnen.

Chat bru'é

Chemincte

Chretienne

Citrononbirne, grofsel

Citron de Sircuz

Sommer

Co rias groise

Confeffelsbirne

Cornelia fchöne

Cuiff-Madame

Dunnegier

Deux fois l'an

Double Calyce, Poir à devx rètes

fleur (Pyrus Communis fl. pleno) panaché

Doyenne grise

blanc longue

Dubbe!de Rict-Peer

Echaffery ou Bezy de Chas-

Epargne, Beau prefent St. Samifon

Epine d'été ou fondanto

\section{d'hiver} musquée

Etrangles. T, de fufse (Kau-

Eyerbirne, Herb? nenbirn)

Sommer ou Colmar

Muskirte Winter

Faux Bonchretien

Fin'or
Autoren|Reifzoit

Quintinye Nov Merz Baum Cat. Dec, Jan. idem

T. Ohftg. Aug. Sept. Chrift July Schuiz Jan. April

T. Obrtg. bis lohanni Knoop. Augint. Duham Jul. Aug.
Baum Cat. Chrift Jul. Oct.

Pom. aust. Aug. Sept.

Quintynie Febr. Merz Duham id id November Diel October Knoop Sept. Oct.

Duham. Nov, Jan. id Jul. Aug. id September id Nov. Jan.

Manger rept. Oct. Sickler Dec. Jan.

Duham. Aug. Sept. Diel Dec. Febr Manger Dec, May Duhuin Augult Forel: 
Birnen.

Forellenbirn

Franchipanne

Friederichs- Birne

Gallusbirne

Geishirtelsbirne

Glockenbirne

Grand-Monarque

Hummelbirne

Hennenbirne

Hirfchbirne

Hafenbirne

Imenbirne

Imperiale á feuille de Chene Inconnue Chenau ou Fondante de Brest
lungfernbirne (Poire de Iutten-Beer

Kronbirne

Louise bonne

Madame, Franz Madame Manfuete ou Solitaire
Marguerite la grofse, Pom-

Marquise me hative la petite

Martin sec

Merveille d'hiver

Meffire Jean

Munduetzbirne, runde

mouillebuuche rond) Muscat petit ou $7 \in$ n gueule allemand

Robert, Poireá la reine d'Ananas
Autoren'Reifzeit

T. Obftg. Nov. Dec. Duham.
Schulz

Baum Cat.

\begin{tabular}{|l|l} 
Diel & September \\
T. Obftg. Oct. Dec.
\end{tabular} Chrift Mierz Moy T. Obitg. Oct. Dec, Baum Cat. September T. Obftg. Auguft

Baum Cat. Oct. Nov.

Duham, Merz Apr.

idem Aug. Sept.

Duham October

Chrif Nierz May

Duham Nov. Dec.

Manger Auguft

Duham September

Baum Cat Auguft

Chrift July

Duham Nov. Dec.

id Nov. Jan.

id November

id Oct. Nov.

T. Obftg. Auguft

Duharn

id Juli

id 
Birnen.

Muscat gros d'été

$$
\text { royale }
$$

Huscateller grofse lange

geblümte

$$
\text { doppelttragetide }
$$

Sommer

Ognoanet, archiduc d'été. Amire roux

Orange d'hiver

$$
\begin{aligned}
& \text { Musquéé } \\
& \text { jaune } \\
& \text { rouge d'été }
\end{aligned}
$$

tulipeé, ou poire aux

Pendar

Paradiesbirne, rheinifche

Parfum d'Août

Paffatutti

Paftoral ou Musette d'au-

Perfik peer

Pfalzgräfin, rothe

Pfundbirne kleine

Poire d'Admiral

d'ange d'hiver

d'Ananas

de Colmar ou Poire

de fer Manne

de foret ou grise bonne Jalvé

de Lansac ou Dauphine de Madelaine ou Citron de Larmes
AutorentReifzeit

T. Obftg. Auguft

Chrift Auguit id

Dec. Jan.

T. Obitg. Auguft

Chrift

Duham Jul, Aug. id Febr. Apr.

id Auguft

Diel September

Duham Auguft

id

Chrift

September

T. Obitg. Oct. Nov.

Duham

Chrift

Auguft

Nov. Dec.

Duham Oct, Dec. Chrift Sept. Oct. id

T. Obrt. September

Chrift October

C. de Char Aug. Sept. idem December Chrift Aug. Sept.

Duhamel Jan. Febr. Manger Dec. Febr, Duham Auguft Baum Cat. Merz April Duham. Dec, Jan. 
Birnen.
Poire de Malthe ou Callat- $\left.\right|^{\text {Autoren }}$ Reifzeit de Naples rosa d'hiver de Prince d'Oignon, Epine rose d'été

de Prêtre

de Prêtre

de St. Pierse

de Picardie

de Londres

de Septemb, le gros

cle Vitry

Fürfliche Tafelbirne au Lard

vierge

Portail (Pfingtthirne)

Present royal de Naples

Querusbirne

Keine d'hiver

Riedbirne (Angobert)

Roi d'été

Royale d'hiver

Rousselet d'hiver

de Rheims

Musqueé

d'été (kleine Sommer Rouis.)

gris

braunrothe Sommer

kleine Muscaseller

Roufseline

Salviati

Sanguinole

Sarazin. Pomme Sarazin

C. de Char. Dec. Jan.

Duhamel Fehr. Merz

Diel Septeniber

Duham. Auguft

idem

id

T. Obitg. $\begin{gathered}\text { Augurt } \\ \text { idem }\end{gathered}$

T. Obftg. Óctober

Chrift

September

Duham.

Conrift

Manger id

Chrift Jan. May

T. Obftg. Merz Apr. Carisruhe

Chr ft Nov Merz

\begin{tabular}{c|c} 
id & October \\
Duham. & Auy. Sept. \\
id & Dec. F. br. \\
id & Fehr. Merz \\
id & Aug. Sept. \\
Diel & September \\
& \\
id & idem \\
id & Octoher \\
id & Auguft
\end{tabular}

T. Obftg. Septer: ber Diel Oct. Nov,

Duham. Auguft id C. de Char I Jahr 


\section{- $84 .-$}

Birne n.

Sarazin de Duhamel

Savoureuse

Schäublerbirne

Schmalzbirne, römifche

Schwarzburger birne

Seilebirne

Stadelbirne

St. Germain ou Iaconnu la-

St. Lezin

Stümpflerbirne

Stumpfling

Sucré vert

d'hoyerswerda

d'hiver
Supreme ou Supreme de

Teton de Venus

Verte longue d'Automne ou Merlé Mouille bouche panaché

d'hiver

Voltmar sche

Virgouleuse(Paradiesbirne) Wälfche - Birne

Wafferbirne (gelbeSommer-

Zuckerbirne Sommer
Autoren|Reif zeit

Duham

I Jahr

Chrift November

Baum Cat. October

Diel Aug. Sept.

Cassel

Baum Cat. October idem

id

Duham

Manger

Baum Cat.

T. Obfg. September

C. de Char. Oct, Nov.

Baum. Cat. October id

Duham

Chrift

id

Duhamel July Áug.

T. ObItg. Jan. May

Duham

id

Henne

Chrift

October id

Dec. April

October id Nov. Jan.

T. Obftg. September

Diel

id id

id 
Aprikosen. Abricot d'Angoumois Alberge blanc d'Hollande, Amande d'Aveline ou Abricot hatif Musqué deNancy, AbricotPeche noir ou abricot d'A. ordinaire panaché de Provence

de Tours Le gros de Portugal

Violet précoce

le gros
Autoren|Reifzeit

Duham Mitte July idem id id id id Anf. July id Mitte July id Ende July id Mitte Aug.

Mayer Ende July Diel Anf. Iuly Mayer Mitte July Duham. id Baum Cat.

Duham. Mitte Aug. id Anf. Aug. Anf. July 


\section{Pfirsiche.}

Abricotée ou admirable jaune, ou la grofse pe-

Admirable rouge

ou Belle de Vitry

Violette

Alberge jaune

Avant peche blanche ou

Bourdine

Montague praecoce

Bellegarde ou Galante

Belle de Tillemont

Brugnon Violet Musqué

\section{natif}

Cardinal Fürftenberg

Chevereufe tardive

Chanceliere

Double Lac

de Troyes ou petite

Swolisch Mignon

Montagne

Grofse Mignonne Incomparable en beauté Murray Nectarine Madeleine blanche

llignonne

rouge ou Macil. de

tardive Courson

Monfrueuse ou Poire rouge de Pomponne Pavie

Monfrin de Camus

Montagne la grofse
Autoren|Reifzeit

Mayer October

Duham Mitte Sept.

Mayer Anf. Aug.

Chrift Anf. July

Duham. MitteSะpt.

Mayer Ende Aug. C. deChart. Ende Sept.

Mayer Anf. Sept.

Chrift Mitte Oct.

Duham Ende Sept. idem Auf. Sept.

Duham Ende Aug. Chrift Anf. Sept. id Ende Aug.

Mayer Mitte Aug. Cat.deChar Ende Aug.

Duham Mitte Aug.

Duham Mitte Aug. id. Oct. Nov.

id Anf. Oct. C. de Char. Ende Sept. T. Obfg. id. 


\section{Pfirsichen,}

Nivette veritable

Newingtons Nectarine Pavie de Newington blanc

Peche Cerise de Malthe

Peche à fleur double

Persique

Pourpreé vineuse tardive

Royale

Sanguinole ou Bete rave Teint doux

Teton de Venus

Violette hative lapetite la grofse
AutorenlReifze it Duham. Ende Sept. Chrift Auguet C.deChart. October

Duham

Chrift Diham Mayer Duham ham Oct. Nov. id Ende Ang. \begin{tabular}{l|l} 
id & Oct. Nov. \\
id & Ende Sept. \\
id & Nitre Oct. \\
id & $\begin{array}{c}\text { Ende Sept. } \\
\text { id }\end{array}$
\end{tabular} \begin{tabular}{l|l} 
id & Oct, Nov. \\
id & Ende Sept. \\
id & Nitre Oct. \\
id & $\begin{array}{c}\text { Ende Sept. } \\
\text { id }\end{array}$
\end{tabular} \begin{tabular}{l|l} 
id & Oct. Nov. \\
id & Ende Sept. \\
id & Nitre Oct. \\
id & $\begin{array}{c}\text { Ende Sept. } \\
\text { id }\end{array}$
\end{tabular} \begin{tabular}{l|l} 
id & Oct, Nov. \\
id & Ende Sept. \\
id & Nitre Oct. \\
id & $\begin{array}{c}\text { Ende Sept. } \\
\text { id }\end{array}$
\end{tabular} id id id
Mitte Aug. Anf. Sept. Mitte Sept. Ende Sept. Anf. Sept. 
Kirschen. Amarelle à longue queue Aurichotte à courte queue

Belle de Choisy

Bigarreau hatif

de May

noir le gros

noir tardif ou de Lory

Cardina'e

rouge le gros

Cerise à bouquet

à fleur double

royale, encanne oul

Chery-Duke

ou la nouvelle d'an-

gleterre

(rrorse Cerise rouge d'Ostheim

tardive ou de la Tous-

saints'

Prunus semper flo-

$$
\text { rens Willd) }
$$

Coeur de pigeon, Bigarreau commun ou belle de Rocmond

Double Natte

Folgerkirsche, holländische

Glaskirsche doppelte

Griotte d'Espagne

de Portugal

$$
\text { la grofse }
$$

de Chaux ou Griotte d'Allemagne
AutorenlReifzeit Baum. Cat Anf. Aug. id.

Chrift Anf. IJuli

Cat.deChar Im Juni

Mayer Ende Juni

Baum Cat. Einde Mai idem Mitte Juli id. September Mayer Mitte Juli Chrift Mayer Ende Juli

Duham

Anî.ईJuli

Cat.deChar id. Chrift

Duham Aug. Oct; 
Kirs chen.
Griotte d'Angleterre la vraie Grofse gobet à courte queue Guigne blanche la grolse

$$
\begin{aligned}
& \text { jaune } \\
& \text { de Sans-Souci }
\end{aligned}
$$

Herzkirsche späthe rothe

$$
\begin{aligned}
& \text { weirse } \\
& \text { rothe }
\end{aligned}
$$

Hildesheimer späthe

Jerusalems-Kirsche

Knorpel- Kirsche

Lauermanns-Kirsche

Leopoldskirsche

Lothkirsche

\section{bunte.}

Maulbeerkirsche

Maykirsche füfse

frühe grofse

Merise à fleur double

Molkenkirsche

Montmorency à couriequeue ou gros Gobet à longue queue

Morelle hative doppelte

Muscat de Prague

Nonnenkirsche

Ochsenherzkirsche

Perlherzkirsche

Pomeranzenkirsche

Prinzenkirsche

Quatre à la livre

Schlotterkirsche

Schwarzbraune frühe knrzstielige
A u toren.|Reifzeit Chrilt

Mayer Mitte Juni idem Ende Juni Baum Cat.

Chrift.

T. Obrg. Ende Aug. Chrift Ende Juli \begin{tabular}{l|c} 
id. & Juli \\
id. & Juni \\
id & Juli \\
id. & id \\
id. & id
\end{tabular}

T. Obftg. Anf. Juli Chrift Anf. Juni T. Obftg. idem Mayer Chrift

\section{Anf. Juli}

Mayer Mitte Juli C. deChart. Ende Juni Chrift

Chrift Ende Juli T. Obitg. Juli Chrift Anf. Juli T. Obftg. Ende Juli \begin{tabular}{|c|c} 
id & Ende Juni \\
Chrift & Ende Juli \\
Car!sruhe & \\
id &
\end{tabular} 


\section{- $93-$}

Kirschen.

Soodkirsche braune rothe

\section{schwarze}

Süfskirsche punctirte

Turkine

Ungarische schwarzo

Van der Natte

Vineuse de Portugal

Wachskirsche gelbe

Weichsel spanische

holländische schwarze

Ludwigsburger

grofse späthe saure

Weinkirsche grose fchwarze
A u torentReifzeit

T. Obftg. Ende Juli id

id

id

Chrift

T. Obftg.

Chrift idem

Chrift

id

id

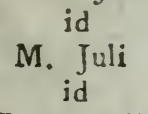

Ende Juli

M. Juli

Ende Juli

M. Juli

id

Septemb.

id 
Pflaumen.

L'Abricotée'

Brizette

rouge

Cueche, Prune d'Allemagne

\section{Verte}

Damas de Maugeron

d'Italie

blanc le gros

Ballon

varieté
de Sept. ou des Vacances

d'Espagne

Misqueé

rouge

do Tours le gros, ou grosse noire hative Damascener späthe schwarze Diapreé violette

\section{rouge \\ blanche}

Drapd'or auMirabelle double Eyerpllaume grüne Grofse luissante ou Damas

\section{Aubert}

noire tardive

Jacinthe, Prune jacin the Jaune hative ou Prune de

Imperiale Violette

$$
\text { Catalogne }
$$

$$
\text { de Sikler }
$$

Isle verte ou Pr. de Savoye,

$$
\text { basse tige }
$$

Mirabelle jaune

$$
\text { rouge }
$$

AutorentReifzeit

Duham Ant. Sept. C. deChart, Ende Aug. id Sept. Oct. Baum Cat. Aug. Oct.

Chrift M. Aug. Duham Ende Aug. id id \begin{tabular}{c|c} 
id & id \\
Baum Cat. id
\end{tabular} id

Duham. Ende Sept. Salzmann September Duham

id

M. Augult Diel Catal.

Duham. Anf. Aug. id
Chrift Anf. Sept. Duham M. Auguft Diel Cat。

Duham

Anf. Sept. id Ende Aug. id Ende Juli Miyer T. Obftg. Anf. Ang. Duham Ane. Sept. id C. de Baum Anf. Aug. 
Pflaumen, Autoren|Reifzeis

Mirabelle tardive ou St. Ca- Quintynie Ende Sept.

Noire

Perdrigon rouge therine

blanc

Pflaume rothe Späthe

Pflaume Cyprische

bunte holländische

Prune sans noyau

Cerise (Mirobalan Mayer)

d'âtriché ou Prune datte

d'Inde blanche

damasquineé

St. Jean

de Monsieur

Virginale

transparente

Reine-Claude la grofse,

Dauphine ou Damas vert

Violette

Schwarze

Royale

de Tours

Zwetsche, Awguft grofse englische

Chrif id

Duham Finde Oct. id Anf. Sept,

T. Obftg. id

Diel Cat. September Chrift Ende Aug. Duham M. Juli

Villars Ende Aug,

Mayer M. Juli

Chrift Anf. Sept.

Salzmann September

Chrift Ende Juni

Duham Juli Aug.

C. deChart, Ende Aug.

Diel Cat. Auguft

Duham id C. deChart. M. Sept,

Chrift Auguft

Duham M. Auguft id Ende Juli

Diel Auguft

Chrift Anf. Aug, Diel Auguft 


$$
\begin{aligned}
& -93- \\
& \text { Wein. }
\end{aligned}
$$

Chasselas de Fontainebleau

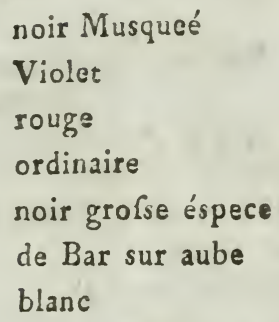

Corinthe blanc sans Pepin

violet

Cornichon violet

$$
\text { beau }
$$

Culotte suisse

Diamant Traube

Klaefner weifser

$$
\text { kleiner }
$$

Muscat d'Alexandrie blane

$$
\begin{aligned}
& \text { blanc } \\
& \text { noir } \\
& \text { violet } \\
& \text { gris }
\end{aligned}
$$

Morillon hatif ou Raisinde la Madelaine

Perltraube frühe

Perl, grofse

Pinot blanc 


\section{Drudéfeller in ber Befareibung.}

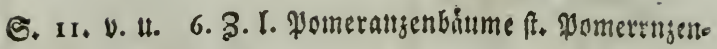
bảumte.

- 17. v. ช. 10. 3. I. TRabaliatti ft. Sinbaliatti.

- 19. v. 0. 7.3. l. ftebenden fi. gebendent.

- 30. v. v. 8. 3. L. Dberbaudireftor ft. Dberdirctor.

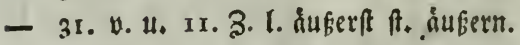

- 32. v. ‥ II. 3. l. ausgelegt fi. angelegt.

- 37. v. 9. 10. 3. 1, Pinus ff, Pinis. 


\section{- 97 -}

\section{Wein,}

Praecoce noir

Raisin de Pulsar

de Maroc

gros de Marseille

de grand Seigneur

Raisin

d'Aspirante

rouge de Bourgogne

gros de Malthe

Toquai

Van de Laan, frühe

Verge blanc

violet

Weifser Burgundez 


\section{Drudélehler in Rataloge.}

5. 32. v. น. 6. 3. I. Juniperus nana ff. J. rana.

- 34. v. u. 5. 3. I. Limodorum altum ft. L. album.

- 44. y. จ. 7. \$. I. Phylica paniculata latifolia fí.

Phylica latifolia.

- 46. H. 47. if bey den Populus- Into Prunus-2tro ten binten immer bas zeichen w ft. arb. gefeçt worden; Diefer febler fommt noch ant mebrern Drten bor, wo ifn aber Der פjo. tanifer leicht fébft verbefiern fant.

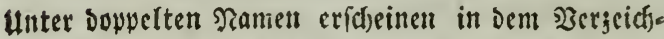
niffe nachftebende Pfiangen, Die bod) nur eine und Dicfelbe Piflange find:

I. Turnefortia hirsutissima und Cestrun paniculatum.

2. Flox sibirica und Flox sabulata.

3. Spielmannia africana uno Lantana africana. 


\section{Druffegler in ber Befdreibung.}

6. 2. 3. 6. von oken liek greber fiatt Frecher

- - 4. von anter l. Eulgingen fr. Enejingen

- 3. - 2. v. u. in ber গote I, arcem ft. arum

- 11. - 9. v. 0. 1. Dberbeimer ft. Sberrheiner

- - 6. v. a. I. pemeranjentaume fi. Tonterenjens

báume

- I6. - 2.4.7. v. 0. 1. Marftall fi. Maríditall

$-17 .-10$ v. 0. I. Rabaliatti fi. Sabaliatti

- 19, .. 7. 0.0 1. Arebenden fi. gebender

- 30. - 8. 0. 0. I. Dberbaudireftor fit. Dberbireftor

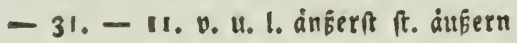

$-32 .-11.0 .0 .1$ ansgelegt fit. angelegt

--18 . v. 0. l. (s) it. (s)

- 37 - 10. v. 0. I. Pinus ft. Pinis

- 33. .. 1. v. u. I. Qinfs it. Piect

- 39. - 1. v. 0. 1. Piedts it. Linfs

- 48. - 13.4. 14. V. 0. 1. Eaale fi. Sande

$-\$ 9 .-3$. V.u. l. Menagerie fi. Drangetie 


ke the $\Rightarrow$

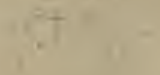

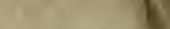

$\frac{1}{3}$

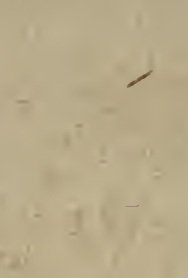

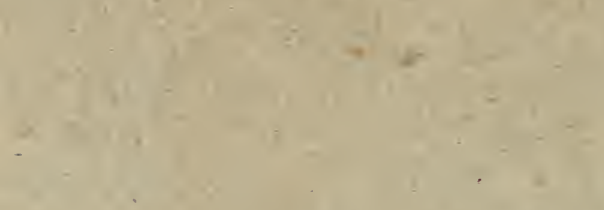

20

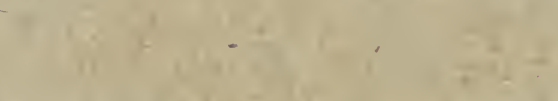

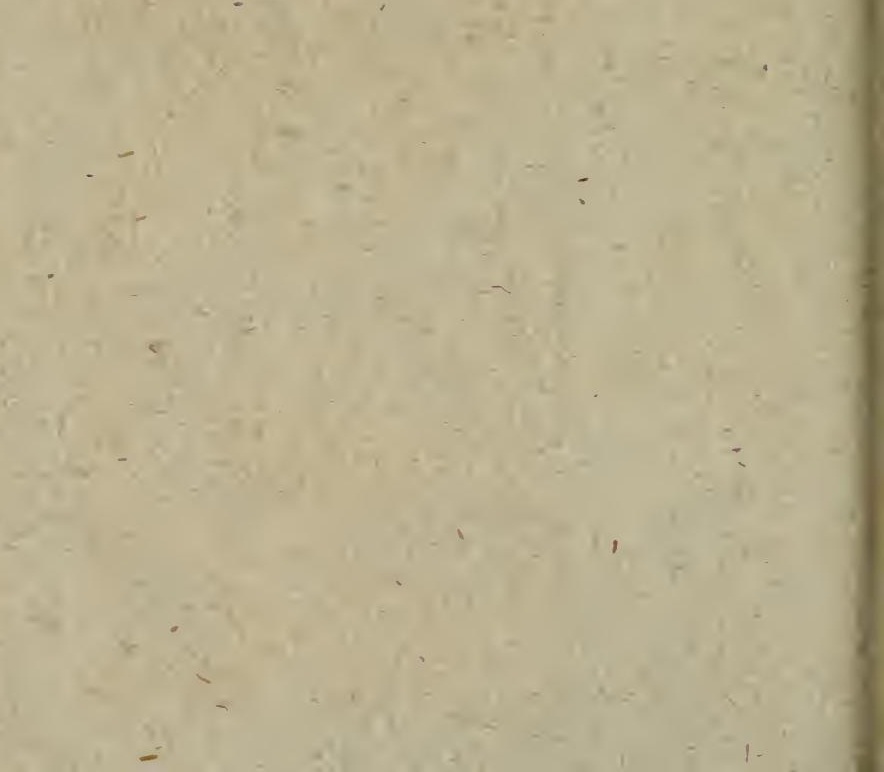


SPECIAL $89-B$
656 
